(W)

update

Cite as

Nano-Micro Lett.

(2020) 12:66

Received: 19 November 2019

Accepted: 2 February 2020

Published online: 28 February 2020

(C) The Author(s) 2020

\section{Two-Dimensional Materials in Large-Areas: Synthesis, Properties and Applications}

\author{
Ali Zavabeti ${ }^{1,2,3} \bowtie$, Azmira Jannat ${ }^{3}$, Li Zhong ${ }^{1,3}$, Azhar Ali Haidry ${ }^{1}$, Zhengjun Yao $^{1}$, \\ Jian Zhen $\mathrm{Ou}^{3}$ 凶 \\ $\square$ Ali Zavabeti, ali.zavabeti@nuaa.edu.cn; Jian Zhen Ou, Jianzhen.ou@ rmit.edu.au \\ 1 College of Materials Science and Technology, Nanjing University of Aeronautics and Astronautics, \\ Nanjing 211100, People's Republic of China \\ 2 Department of Chemical Engineering, The University of Melbourne, Parkville, VIC 3010, Australia \\ 3 School of Engineering, RMIT University, Melbourne, VIC 3000, Australia
}

\title{
HIGHLIGHTS
}

- Two-dimensional materials including TMDCs, hBN, graphene, non-layered compounds, black phosphorous, Xenes and other emerging materials with large lateral dimensions exceeding a hundred micrometres are summarised detailing their synthetic strategies.

- Crystal quality optimisations and defect engineering are discussed for large-area two-dimensional materials synthesis.

- Electronics and optoelectronics applications enabled by large-area two-dimensional materials are explored.

\begin{abstract}
Large-area and high-quality two-dimensional crystals are the basis for the development of the next-generation electronic and optical devices. The synthesis of two-dimensional materials in wafer scales is the first critical step for future technology uptake by the industries; however, currently presented as a significant challenge. Substantial efforts have been devoted to producing atomically thin two-dimensional materials with large lateral dimensions, controllable and uniform thicknesses, large crystal domains and minimum defects. In this review, recent advances in synthetic routes to obtain high-quality two-dimensional crystals with lateral sizes exceeding a hundred micrometres are outlined. Applications of the achieved large-area two-dimensional crystals in electronics and optoelectronics are summarised, and advantages and disadvantages of each approach considering ease of the synthesis, defects, grain sizes and uniformity are discussed.
\end{abstract}

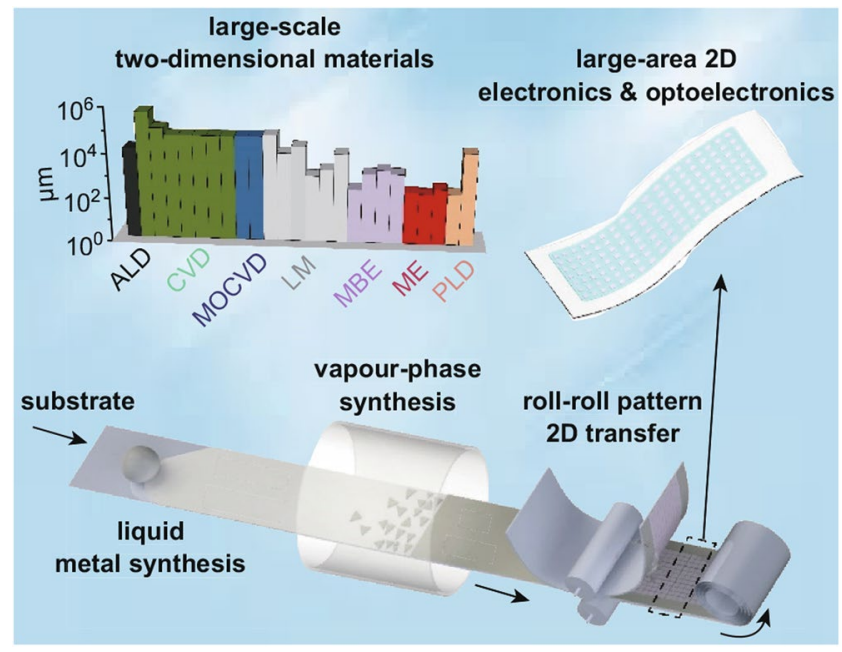

KEYWORDS Two-dimensional materials; Large-area; Electronics; Optoelectronics; Defect engineering 


\section{Introduction}

Synthesis of high-quality and atomically thin materials in large areas is a subject of an intensive and ongoing investigation. Controllable growth of ultrathin two-dimensional (2D) materials in large areas enables design and integration of electronics devices with complex components, providing enhanced interfaces for optical and heterostructure devices [1]. Detrimental consequences on device performances are due to the non-uniformity and formation of defects in 2D crystals during synthesis. The thickness of $2 \mathrm{D}$ crystals is influential in optical, vibrational and electronic properties. Therefore, the control in thickness and uniformity of synthesis is instrumental for the reliability of device performance [2-7]. According to laws of thermodynamics, synthesis at temperatures above $0 \mathrm{~K}$ will result in the formation of defects in all crystals $[8,9]$. Controllability in both thicknesses and defects are primarily managed by engineering the reaction kinetics and thermodynamics conditions during the synthesis process. Here, we report on the recent advancements in the synthesis of large-area 2D materials including transition metal dichalcogenides (TMDCs), hBN, emerging materials (black phosphorous, Xenes, bismuth compounds), non-layered materials and graphene. Here, we refer to "large-area" as lateral dimensions larger than $100 \mu \mathrm{m}$ and "ultra-thin" with thicknesses of smaller than $10 \mathrm{~nm}$.

Advantages and disadvantages of synthetic approaches considering challenges in thickness control and the resultant crystal quality are discussed by characterising the defects, disorders and grain sizes. Finally, the overview of applications in electronics and optoelectronics exploited by printing large-area materials in 2D are provided.

\section{Record Lateral Dimensions}

The quest to enhance lateral and crystal domain sizes is depicted in Fig. 1a, b. The first exfoliated graphene monolayer by Novoselov et al. in 2004 and consequently, several TMDCs such as $\mathrm{MoS}_{2}$ and $\mathrm{NbSe}_{2}$ in 2005 isolated in 2D below $100 \mu \mathrm{m}$ in lateral dimensions [10]. As illustrated in Fig. 1a, these three materials' dimensions have expanded to more than three orders of magnitude by chemical vapour deposition (CVD) synthesis [11]. Many emerging materials, such as borophene and Mxene, are yet to be realised larger than a hundred microns (Fig. 1a) [12]. Emergence of liquid metal (LM) synthesis is shown by arrows to the synthesis of $\mathrm{GaS}$ and 2D oxides by using liquid metals as a reaction solvent (Fig. 1a) [13, 14]. Metal oxides and hydroxides are an important category of materials with versatile and unique optical and electronic characteristics, which Sasaki group has pioneered synthesis of these materials including titanium oxide, manganese oxide and niobium oxides in suspensions with the largest reported dimensions of tens of micrometres for a $2 \mathrm{D}$ stoichiometry of titanium oxides $\mathrm{Ti}_{0.87} \mathrm{O}_{2}^{0.52-}$ [72] .

Figure $1 \mathrm{~b}$ represents $2 \mathrm{D}$ materials synthesised in large lateral dimensions exceeding $100 \mu \mathrm{m}$ and thickness of below $10 \mathrm{~nm}$. Several novel materials such as borophene and Mxene and novel methods including soft chemical processes are added to Fig. 1b. Synthesis methods for the novel materials are expected to continue to be optimised. Crystal domain sizes for many of the included materials in Fig. 1b have not been reported or optimised. As presented in Fig. 2c, when considering crystal domain sizes, the list of large-area printed materials reduces to CVD, ME and MBE methods.

Altogether, the CVD method holds promise for the synthesis of many 2D materials with large crystal domains including TMDCs, graphene and hBN (Fig. 1b, c) [11, 17-19].

Material categories and different synthesis routes to achieve them in the 2D large-area are detailed in the following section.

\section{Large-Area 2D Materials Synthesis}

Extensive efforts have been dedicated to the synthesis of atomically thin materials with laterally large dimensions. Various approaches are investigated which can be typically assorted into two categories which entail top-down and bottom-up techniques. The most notable top-down approaches are exfoliation techniques, including liquid exfoliation and mechanical cleavage. Liquid exfoliation presents challenges in balancing produced quality vs largearea yield of 2D flakes. Agglomerations, limited-sized 2D sheets with arbitrary shapes and random distribution on substrates, have been drawbacks of liquid-phase exfoliation [73, 74], Mechanical exfoliations, however, have been a benchmark for high-quality exfoliated 2D sheets, and innovative approaches have enhanced the lateral size and controllability in patterned transfer [20-24]. However, 

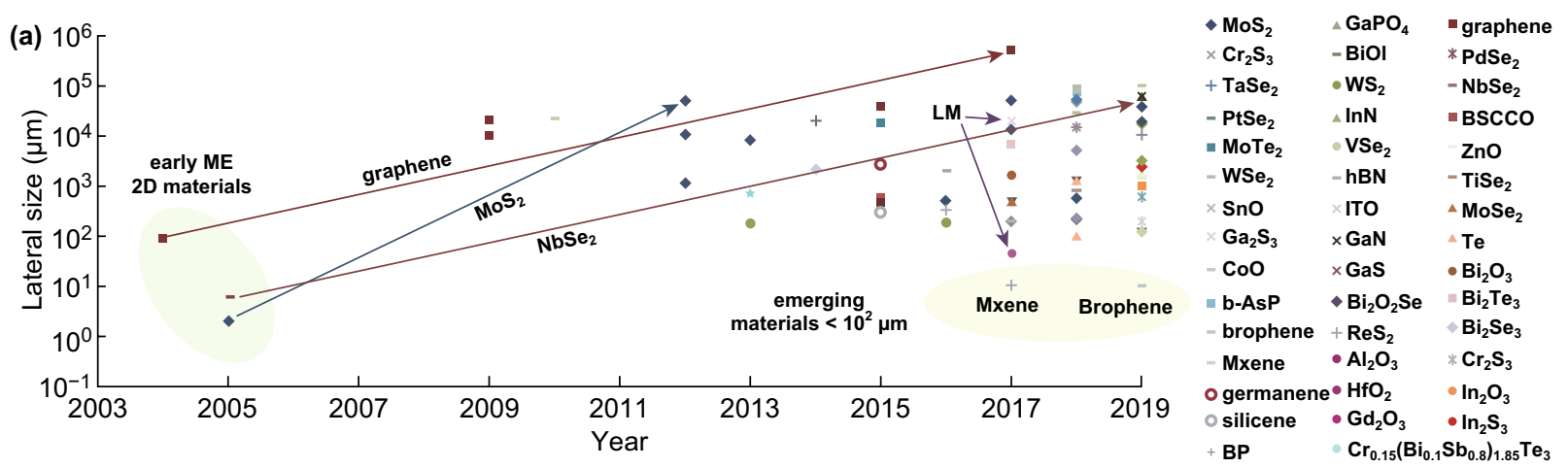

(b)

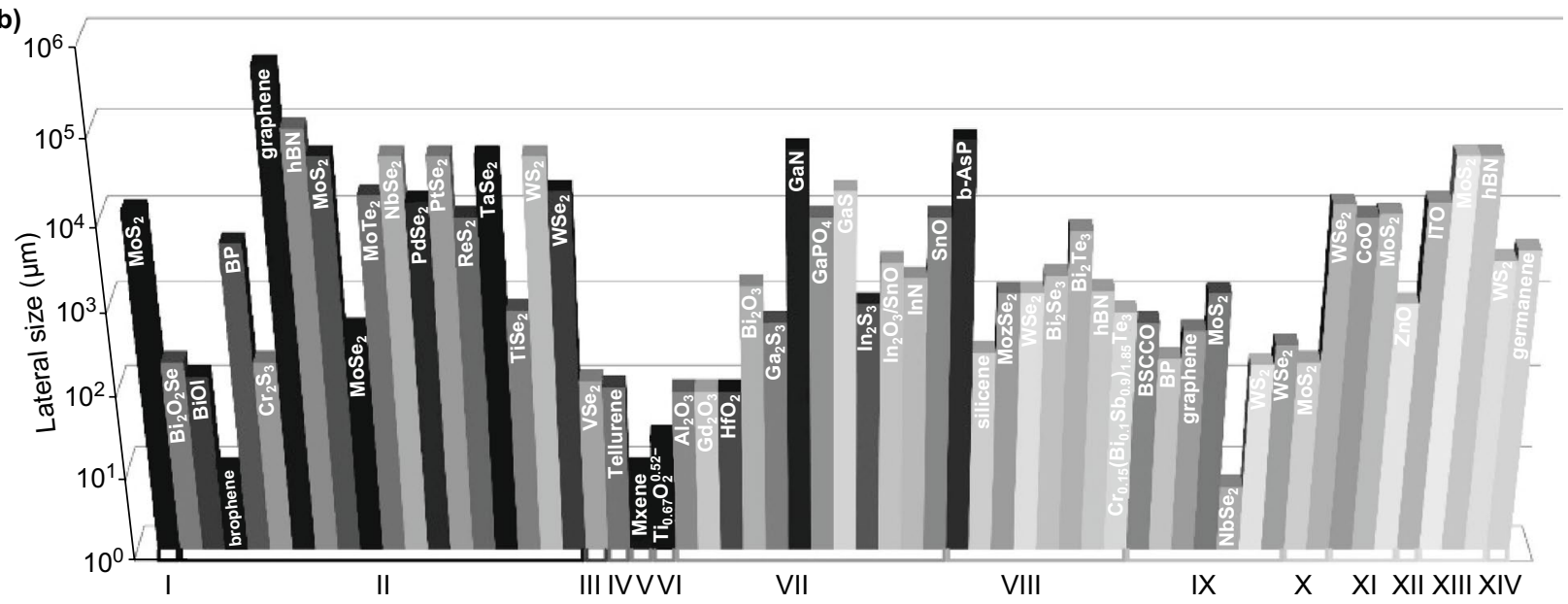

(c)

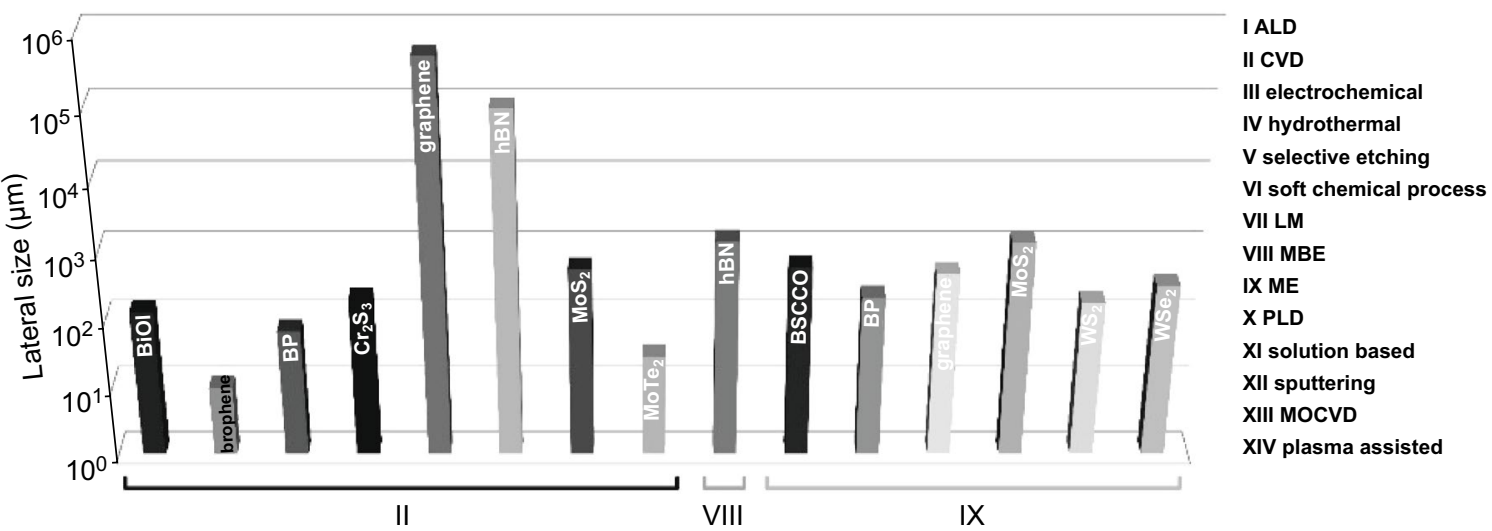

Fig. 1 Lateral dimensions of 2D materials. a Evolution of lateral sizes from ME to CVD synthesis are shown for $\mathrm{MoS}_{2}$, NbSe $\mathrm{N}_{2}$ and graphene with arrows. Emerging 2D materials below $100 \mu \mathrm{m}$ in lateral dimensions is highlighted in yellow. b Lateral dimensions are elucidated for each 2D material derived from different synthesis routes [2, 3, 7, 10-71]. c Record lateral dimensions achieved as a single crystal [3, 11, 17, 20, 21, $23,24,38,41,46,57,58,66]$. (Color figure online)

bottom-up approaches, such as CVD, prevail as the most potent technique so far. This method is industry-relevant and applicable to many materials with ease of operation. However, numerous operating parameters require thorough knowledge and engineering to obtain high-quality crystals.
Key metrics include (1) amounts, morphologies and stoichiometries of the precursors [5, 25], (2) temperature of the precursors and substrate [5, 25, 26], (3) location and distance between of inlet, precursors and substrate [4], (4) pressure of the reaction chamber [5], and (5) carrier gas types and 


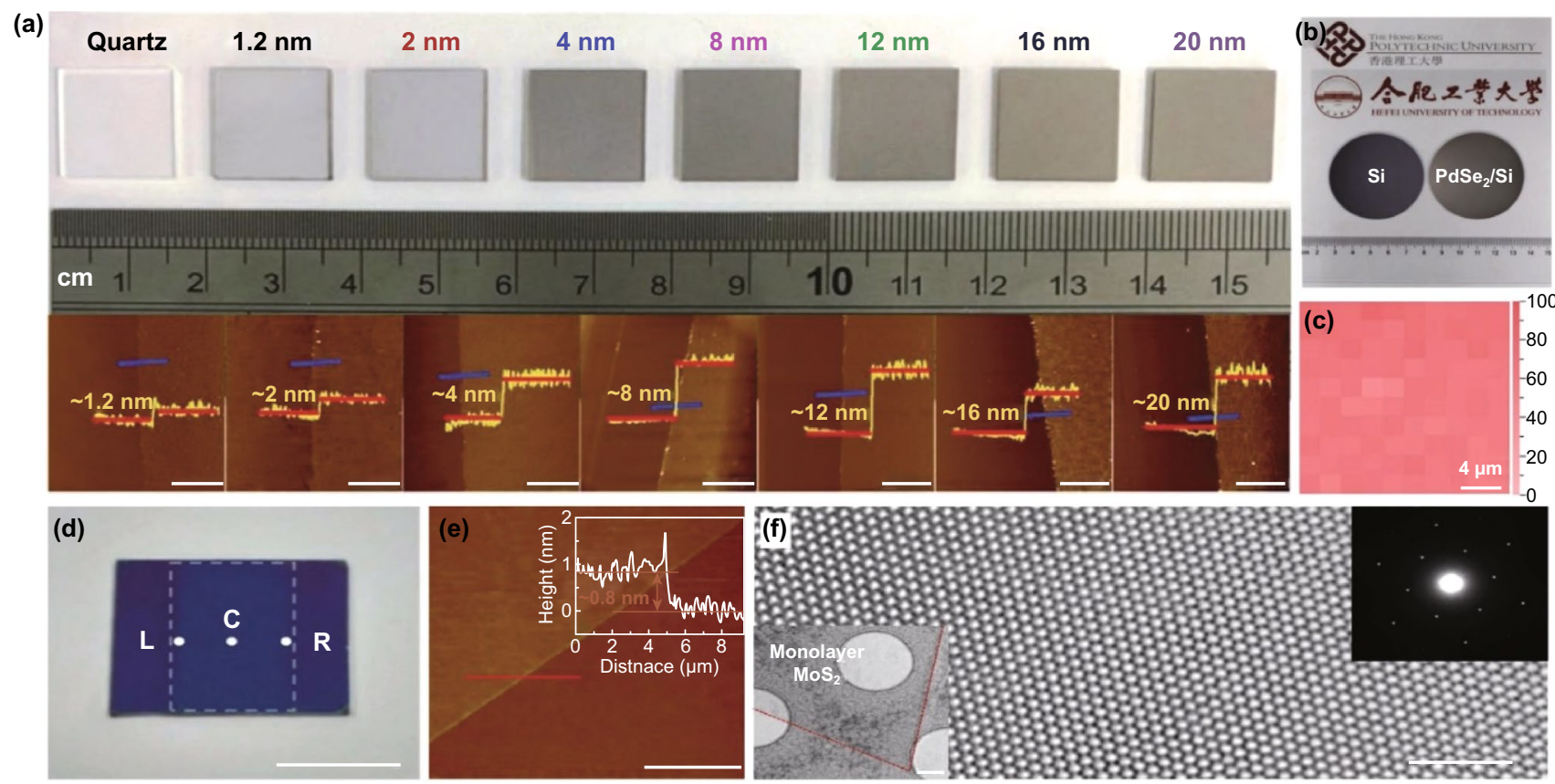

Fig. 2 Large-area CVD synthesised TMDCs. a Optical images of CVD synthesised PdSe 2 (top) and corresponding AFM height profiles (bottom) showing the obtained tuned thicknesses. b Optical image of a 2D PdSe 2 wafer-sized synthesised product with a plain Si substrate. $\mathbf{c}$ Raman intensity mapping of a $20 \times 20 \mu \mathrm{m}^{2}$ area indicating good film uniformity. Adapted with permission from Ref. [28]. Copyright 2019, Wiley. d Optical image of a CVD grown $\mathrm{MoS}_{2}$ grown from oxide precursor from an engineered configuration. e AFM image and the inset thickness profile corresponds to a $\mathrm{MoS}_{2}$ monolayer. f High-resolution transmission electron microscope (HRTEM) image from left inset showing highly quality crystal. The right inset shows the selected area diffraction pattern. Adapted with permission from Ref. [25]. Copyright 2017, Wiley. Scale bars are $2 \mu \mathrm{m}$ (a bottom), $1 \mathrm{~cm}(\mathbf{d}), 10 \mu \mathrm{m}(\mathbf{e}), 2 \mathrm{~nm}(\mathbf{f})$

flow rates $[4,27,75,76],(6)$ type and preconditioning methods of the substrate $[17,18,76,77]$. Engineering and tuning these parameters for the synthesis of each material will enhance controlling nucleation and growth rates leading to more homogenous growth with fewer defects and large 2D sheet sizes. Generally, a balance between precursor mass flux rates and materials growth rate should be established [78] to minimise the nucleation rate initially and maximise the growth rates afterwards.

The synthesis routes are firstly discussed for TMDCs, which present as a promising category of semiconductors with several demonstrated optoelectronics applications. Recently, synthesis of 2D hexagonal boron nitride (hBN) has made a significant enhancement in crystal size which is explored in detail followed by emerging materials that have been produced in large lateral sizes with intriguing properties such as black phosphorus (BP) and 2D Xenes. Progress in the synthesis of bismuth compounds as promising materials for topological insulators is discussed. Most materials are not intrinsically layered and present with challenges to achieve them as 2D using conventional exfoliation or vapour phase methods. However, the emergence of novel synthesis routes has provided them as stratified 2D layers which are presented in this review. Finally, graphene synthesis is discussed. Despite being gapless, large-area synthesis of graphene as the most popular 2D material can offer insights into the large-area synthesis of other semiconducting 2D materials.

\subsection{TMDCs}

TMDCs are a promising class of materials for next-generation electronics and optoelectronics due to their excellent electronic and optical properties [79]. CVD is the most comprehensively studied technique. Depositing the metal precursors before chalcogenisation results in the production of centimetre-scale atomically thin and uniform crystals of $\mathrm{NbSe}_{2}$ [2] and $\mathrm{PdSe}_{2}$ (Fig. 2a-c) [28]. Grain boundary sizes of synthesised $\mathrm{NbSe}_{2}$ were in orders of few nanometers including the tilt grain boundary defects of 5-7 pair interlinks [2]. The quality of the precursor is a critical factor in 
achieving a balance between nucleation and growth rates for maximising produced domain size [25]. Taking $\mathrm{MoS}_{2}$ as an example, $\mathrm{MoO}_{3}$ thin film as the precursor was deposited first using a solution-processed method. Evaporation of $\mathrm{MoO}_{3}$ thin film located above the target substrate at $800{ }^{\circ} \mathrm{C}$ reduced the nucleation density and produced single-crystal domains of up to $500 \mu \mathrm{m}$ (Fig. 2d-f) [25]. On the contrary, direct sulphurisation of bulk Mo foil results in highly defective $\mathrm{MoS}_{2}$ [80]. Enhanced chalcogenisation is commonly achieved by using $\mathrm{H}_{2}$ in addition to an inert gas such as Ar in carrier gas mixture. Mixing $\mathrm{H}_{2}$ in the carrier gas is not required during the CVD synthesis. However, $\mathrm{H}_{2}$ gas assists as a reducer of the oxide precursors during the chalcogenisation process, especially for a less reactive chalcogen precursor such as Se. High crystalline quality $2 \mathrm{D} \mathrm{WSe}_{2}$ is grown in centimetres at $850{ }^{\circ} \mathrm{C}$ using powder precursors and introducing $\mathrm{H}_{2}$ gas for activation of the selenisation process [27]. In addition to the enhancement of crystal quality, uniformity as another important quality indicator that can be improved through adjusting each of the CVD parameters including temperature gradient, confined space, precursor amount and distance between precursor and substrate [26, 29, 30, 81-83]. For instance, multi-temperature zone configuration is reported as an optimisation approach [26]. Using this strategy, Lan et al. [26] produced large-area uniform $\mathrm{WS}_{2}$ monolayers. Centimetre-sized 2D $\mathrm{WTe}_{2}$ with uniform thickness was also synthesised in three-zone temperature CVD system. The thickness was effectively controlled by $\mathrm{WCl}_{3}$ precursor amount and distance between precursor and substrate [5]. Uniformity in CVD synthesis of 2D TMDCs can also be enhanced by minimising the gradient of reactant across the target substrate. The gradient of the reactant was reduced by using a confined space of an inner tube to reduce gas velocity [4]. Using this technique, Guo et al. [4] synthesised centimetre-scale $2 \mathrm{D} \mathrm{ReS}$, with uniform and controllable thickness. In addition to enhancement in uniformity and reduction in defects, the CVD process can offer growth of selective phases. Zhou et al. [3] used CVD method to selectively grow two distinct phases of $\mathrm{MoTe}_{2}$, i.e. $2 \mathrm{H}$ and $1 \mathrm{~T}$ depending on the oxidisation state of the Mo precursor used, resulting in high phase purity and uniformity. Recently, noble transition metal dichalcogenides such as $\mathrm{PtSe}_{2}$ have also been synthesised and become available in large areas [84]. Using the CVD process, Wagner et al. [84] have grown large-area 2D $\mathrm{PtSe}_{2}$, however, with nanometre-sized grains. As a common practice, the CVD grown atomically thin layers are required to be transferred to the desired substrate or to be stacked vertically as heterostructures. Shim et al. [23] discovered a universal method of layer-resolved splitting (LRS) technique to transfer uniform and continuous monolayers of $\mathrm{WS}_{2}, \mathrm{WSe}_{2}, \mathrm{MoS}_{2}$ and $\mathrm{MoSe}_{2}$ with $5 \mathrm{~cm}$ diameters. Growth of large-area emerging TMDCs for applications in quantum physics including charge density wave (CDW) order enhancements has also been realised by CVD methods. $\mathrm{TiSe}_{2}$ and $\mathrm{TaSe}_{2}$ monolayers with areas of $5 \times 10^{5} \mu \mathrm{m}^{2}$ and wafer-scale, respectively, have been synthesised featuring CDW enhancement [31, 32].

In addition to CVD, several other methods are used for the synthesis of TMDCs. Pulse laser deposition (PLD) is recently reported to produce centimetre-sized $\mathrm{MoS}_{2}$ with precise thickness control enabling the fundamental study of thicknessdependent photoresponce of high-quality $2 \mathrm{D} \mathrm{MoS}_{2}$ [7] Similarly, wafer-scale 2D WSe ${ }_{2}$ obtained PLD method is shown to provide defined control in thicknesses and to produce uniform $2 \mathrm{D}$ sheets (Fig. 3a-c) [33]. Large-area $\mathrm{MoS}_{2}$ has been prepared by control of oxide nucleation and growth using thermal and plasma-enhanced ALD (PEALD) following with sulphidation step [34]. Keller et al. [34] explored the crystal quality optimisation by varying sulphidation temperatures, treatment with piranha and multi-step annealing processes (Fig. 4a-c). In the top-down gold-mediated mechanical exfoliation (ME) approach, Javey et al. [24] isolated monolayers of TMDCs, including $\mathrm{MoS}_{2}$ as an example resulting in single crystals with flake lateral dimensions of up to $500 \mu \mathrm{m}$. The schematic is shown in Fig. 4d containing steps 0-6. During this process, gold is evaporated onto a TMDC bulk crystal. As gold has a strong binding affinity towards chalcogens (particularly sulphur), the TMDC top layer can be delaminated together with the gold layer when it is peeled off. Later, gold is etched away, leaving a large-area TMDC monolayer behind [24]. This method is recently extended to produce spatially controlled exfoliation method for TMDCs such as $\mathrm{WS}_{2}$ and $\mathrm{MoS}_{2}$ [86] and reported separately for Mo- and W-based chalcogenides as well as GaSe [85]. Using this method, Velický et al. [85] exfoliated centimetre-sized monolayers from bulk crystals, enhancing the size of flake and feasibility of ME for large-scale production of TMDCs (Fig. 4e-h). It has been demonstrated that the gold-mediated exfoliation is sensitive to air exposure due to the weakening of vdW forces that are used for exfoliation (Fig. 4e-h) [85]. Mechanical shaking is demonstrated to produce single-crystal monolayer $1 \mathrm{~T}-\mathrm{TaS}_{2}$ with lateral sizes exceeding $100 \mu \mathrm{m}$. This method produces large monolayers 

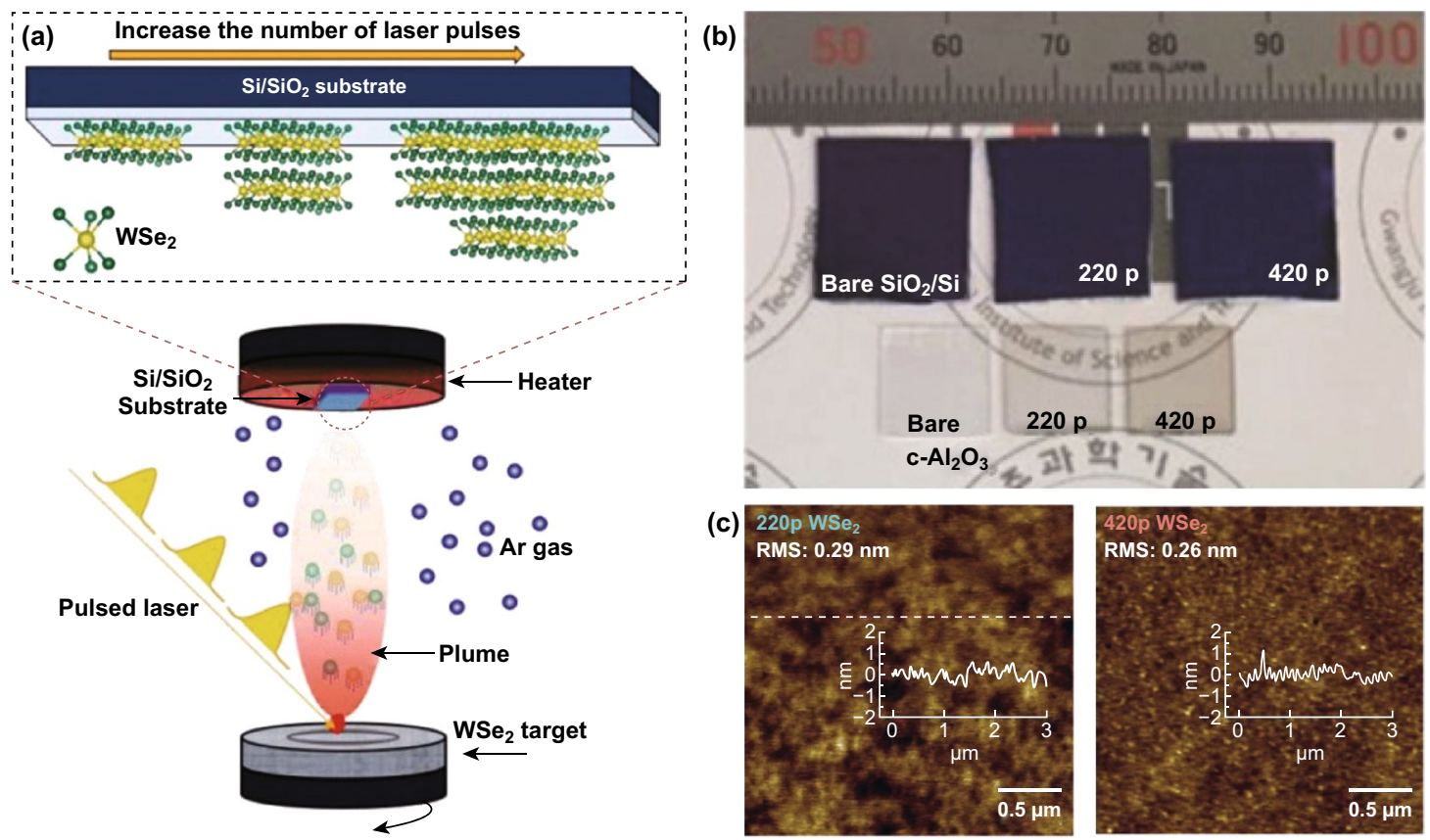

Fig. 3 Large-area synthesised TMDCs by PLD. a Schematic illustration of deposition of WSe $\mathrm{W}_{2}$ by PLD. b Optical images of as-grown WSe thin films on $\mathrm{SiO}_{2} / \mathrm{Si}$ and c $\mathrm{Al}_{2} \mathrm{O}_{3}$ substrates. $\mathbf{c}$ Typical AFM images with a height profile of the $220 \mathrm{p} \mathrm{WSe} \mathrm{W}_{2}$ and $420 \mathrm{p} \mathrm{WSe}{ }_{2}$. Adapted with permission from Ref. [33]. Copyright 2018, Wiley

with manual shaking of $\mathrm{Li}$ intercalated crystals for a few seconds and can potentially be expanded to other TMDCs [87].

This section presents achievement of the large-area highquality TMDCs crystals readily available to be incorporated into practical industrial applications. Many of these methods investigate the growth or isolation of single TMDCs; however, further, development is needed to produce heterojunctions and Janus structures in large-scale as both of these two types of structures are of great interest for high-performance electronic and optical applications [88-90]. Enlarging the overlapping areas for these structures augments their performances by providing larger effective areas. Heterojunctions may be achieved in CVD processes by separation of the precursors and placing them into separate chambers. Then, opening and closing outlets sequentially multiple times during the growth step can produce larger effective lateral heterojunction areas.

\section{$3.2 \mathrm{hBN}$}

$\mathrm{hBN}$ has been widely investigated in fundamental science and used for device applications as an insulator, gate-dielectric, passivation layer, tunnelling layers, contact resistance, charge fluctuation reduction and Coulomb drag [91]. There are many recent reports on the synthesis of high-quality hBN on a wafer-scale $[17,18,35,75-77$, 92] focusing on the minimisation of the structural defects and grain boundaries which impedes high-performance electronics due to charge scattering and trap sites. Similar to TMDC, CVD is still the most powerful synthesis route for producing large-area $\mathrm{hBN}$ with large grain sizes and minimum grain boundary formation [17, 35, 36, 77, 93].

Importance in underlying substrate crystals in CVD growth such as $\mathrm{Cu}, \mathrm{Cu}-\mathrm{Ni}$ alloy and $\mathrm{Fe}$ foils has been known to enable large-area growth of hBN, however, previously resulted in the formation of a significant amount of wrinkles and grain boundaries [36, 94]. Wang et al. explored the effect of the substrate crystal symmetry on growing large-area crystal domains with reduced defects [17]. It is found that the $\mathrm{Cu}(110)$ substrate with a lower order of symmetry than that of hBN (with three orders of symmetry) providing $100 \mathrm{~cm}^{2}$ single-crystal domains [17]. The framework enabled unidirectional growth of large and uniform monolayers of hBN with highly aligned nucleation and domain growth guided by substrate crystal edge-coupling phenomena [17]. $\mathrm{hBN}$ is also shown to form circular grains on liquid metals compared to triangles on 

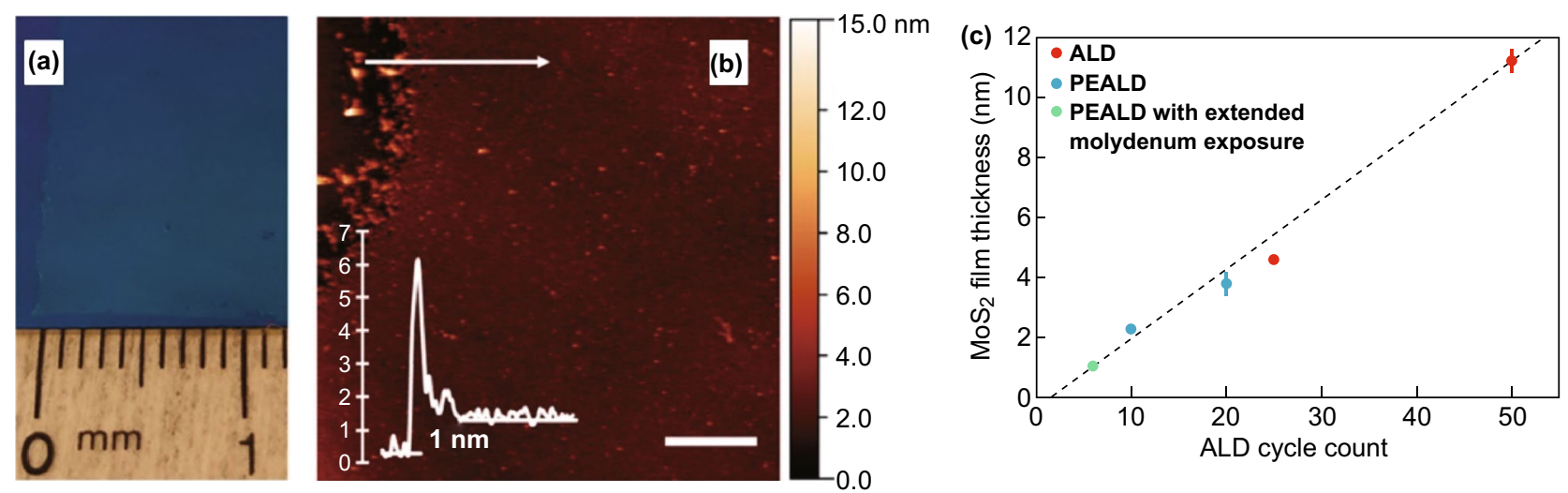

(d)
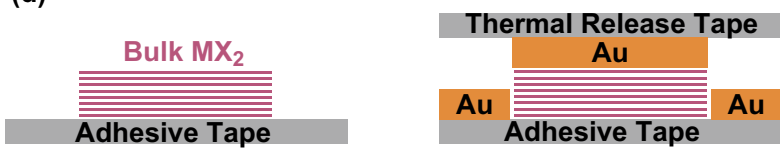

0. Bulk $M X_{2}$ on a tape

1. Evaporate Au and contact thermal tape

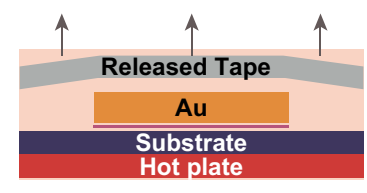

4. Thermally release tape
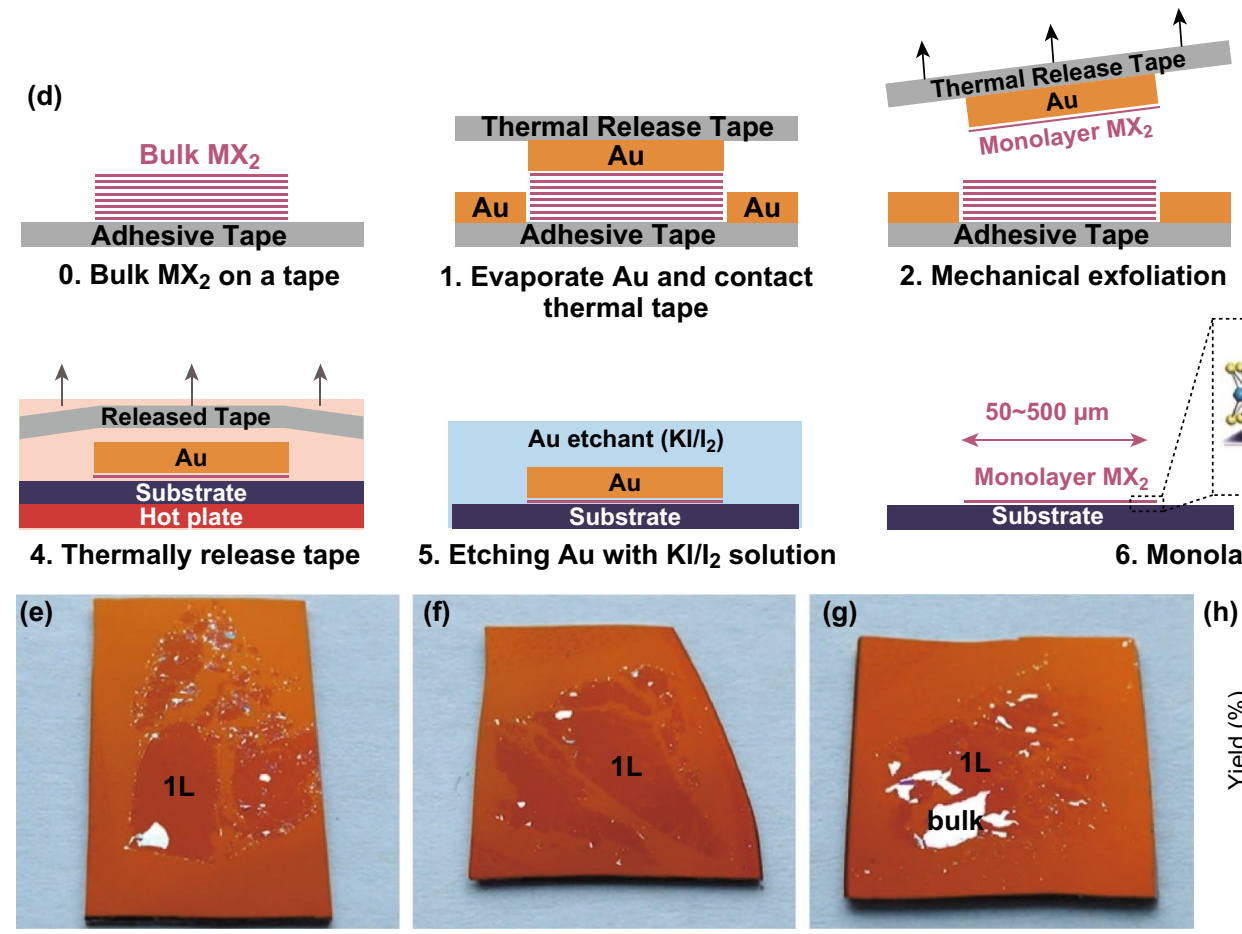

2. Mechanical exfoliation
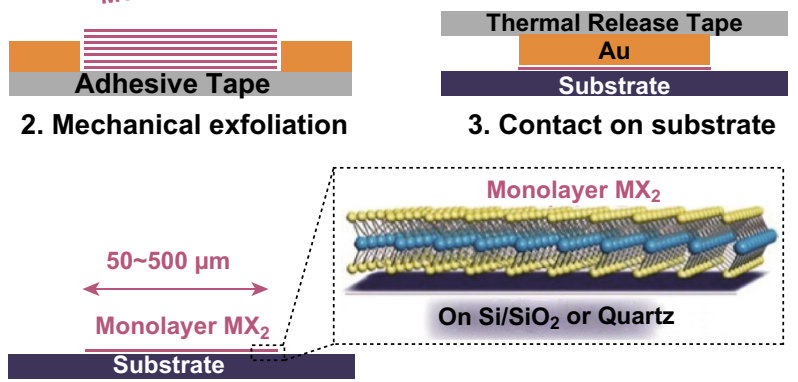

5. Etching Au with $\mathrm{KI} / \mathrm{I}_{2}$ solution

6. Monolayer on substrate
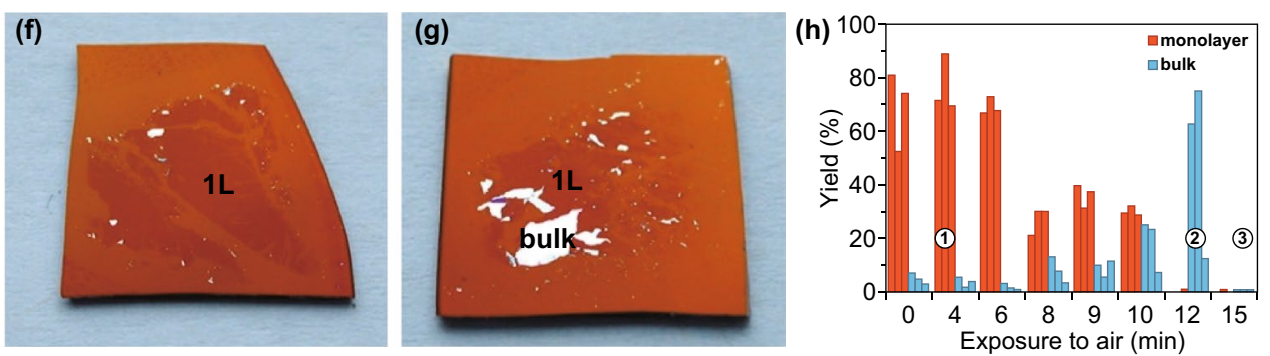

Fig. 4 Large-area synthesised TMDCs by ALD, PLD and ME. a Optical image of 2D $\mathrm{MoS}_{2}$ on $300 \mathrm{~nm} \mathrm{SiO} / 2$ Si substrate showing centimetrescale uniformity achieved with ALD and the post-sulfidation process. b AFM image with a height profile of monolayer MoS ${ }_{2}$. Error bar $^{2}$ diagram of the thickness of $\mathrm{MoS}_{2}$ film during ALD and PEALD process. Adapted with permission from Ref. [34]. Copyright 2017 American Chemical Society. d Schematic illustration of the Au exfoliation process. e-g Optical images of a large-scale $\mathrm{MoS}_{2}$ on $7.5 \mathrm{~nm}$ Au at different periods after the Au exposure to air flakes. h Histogram of the monolayer (red) and bulk (blue) yields at different times. Adapted with permission from Ref. [85]. Copyright 2018 American Chemical Society. (Color figure online)

solid substrates. Large-area single-crystal hBN was grown on liquid $\mathrm{Au}[35]$ which provides a flat surface and allows rotations and alignments, utilising attractive Coulomb interactions between $\mathrm{B}$ and $\mathrm{N}$ atoms (Fig. 5) [35]. The similar phenomena of crystal self-alignment are witnessed on liquid $\mathrm{Cu}[76]$.

Other engineering attempts to enhance quality or thickness control of large-area hBN growth during CVD synthesis include layer growth controlled by cooling rates [96] and the removal of oxygen from the reaction chamber [75]. Stitching of defects in $\mathrm{hBN}$ has been demonstrated by Cui et al. [97] to provide a larger effective area after synthesis. The stitching process entails selective ALD deposition of LiF on defects and grain boundary sites of hBN which produced chemically and mechanically stable hybrids for electrochemical Li plating [97]. Metal-organic 


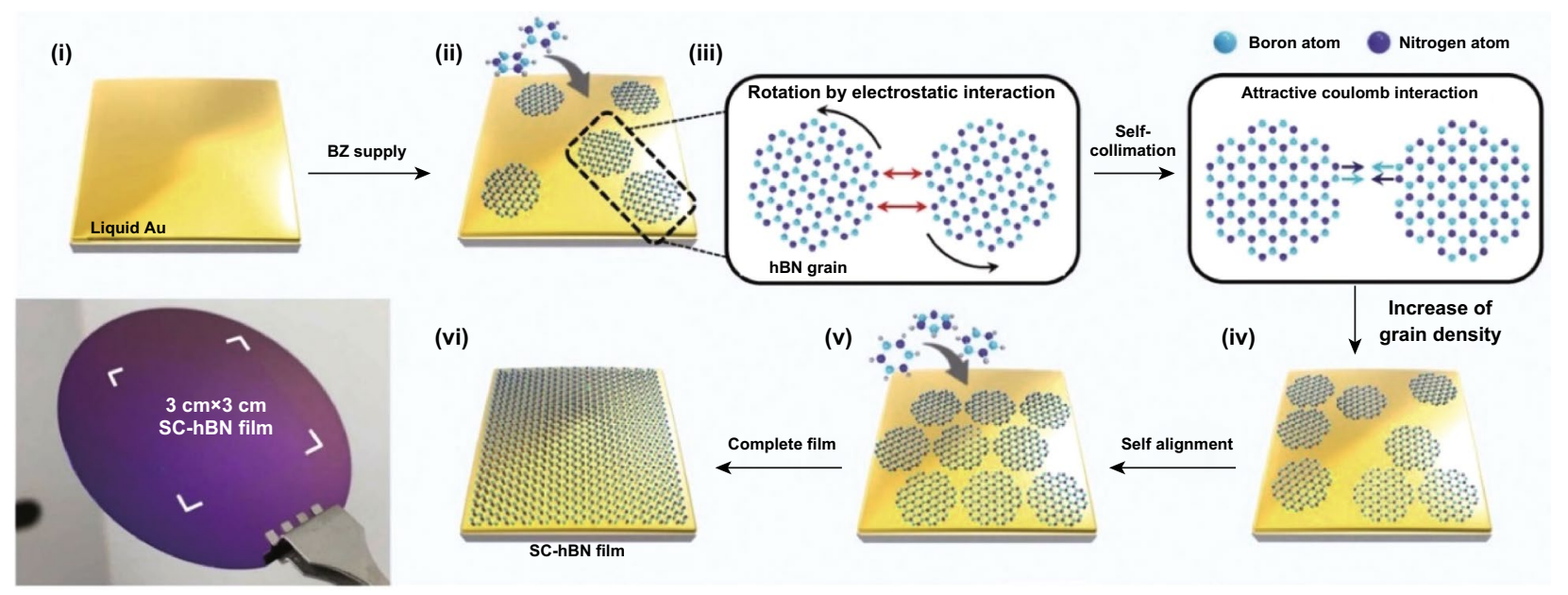

Fig. 5 Large-area CVD synthesised hBN. Sequential schematic of CVD single-crystal hBN growth of the self-collimated circular grains (i-vi). Grains rotate and align due to Coulomb interactions to form a single crystal, as shown in the photograph. Adapted with permission from Ref. [95]. Copyright 2019, Wiley

chemical vapour deposition (MOCVD) framework also offers a wafer-scale synthesis on $\mathrm{Ni}$ (111) substrates with sub-nanometer roughness of $0.605 \mathrm{~nm}$; however, with average grain sizes of $75 \mu \mathrm{m}$ [37]. Other than CVD and MOCVD, hBN has been synthesised by plasma-enhanced ALD [98] yet amorphous with a relatively large thickness of $20 \mathrm{~nm}$ [98]. After synthesis, the grown layers require transferring to the desired substrate. A reliable transfer method ensuring the integrity of large-area 2D hBN remains a challenge. Cun et al. transferred wafer-scale (4 inches) single-crystal hBN with a reliable performance involving a two-step protocol of electrochemical treatment and hydrogen bubbling [18]. The previously explained LRS transfer method has been used to transfer large-area hBN [23].

The synthesis of large-area monolayers of single-crystal hBN has undoubtedly been achieved. However, the methods are enabled by substrate engineering. Since hBN is an insulating material and primarily used in conjunction with other 2D materials as capping or passivating layers, either direct deposition or reliable transfer methods are necessary to be shown for each of the synthesis methods. Similar to liquid metal mechanical transfer methods [14], transfer of the hBN sheets from the surface of liquid Au should be trialled [35]. Possibility of substituting liquid $\mathrm{Au}$ as a substrate with other liquid metals under ultra-high vacuum to avoid oxide and contamination formations should be explored to reduce the working temperatures and costs of the liquid metals.

\subsection{Emerging Materials}

\subsubsection{Black Phosphorus}

Black phosphorus (BP) has high motilities in room temperature with tunable bandgap featuring intriguing properties to be incorporated in device applications [38]. Large-area stratified crystals of black phosphorous with lateral dimensions of up to $600 \mu \mathrm{m}$ were synthesised using a custom configuration. Li et al. used red phosphorous powder as a precursor and deposited on a sapphire substrate. Then, red phosphorous films were firstly covered by $\mathrm{hBN}$ and then followed by annealing at $700{ }^{\circ} \mathrm{C}$ in $1.5 \mathrm{GPa}$ pressure to convert to BP. The thermodynamics was engineered to ensure hBN crystal remained unchanged and operating temperatures were below the melting point of BP. Domain sizes range from 40 to $70 \mu \mathrm{m}$ with mobility of $\sim 200 \mathrm{~cm}^{2} \mathrm{~V}^{-1} \mathrm{~s}^{-1}$ at $90 \mathrm{~K}$ [38]. Similar to TMDCs [24], BP was exfoliated using a top-down approach through the gold-mediated exfoliation with lateral sizes exceeding $100 \mu \mathrm{m}$ (Fig. 6a-c) [21]. However, this method resulted in sheet breakages, random distribution of flakes and less control in thicknesses [21]. Other compounds of BP have been synthesised in wafer-scale. Black arsenic-phosphorus (b-AsP) sheets with thicknesses of 6-9 $\mathrm{nm}$ are synthesised at wafer-scale using molecular beam deposition (MBD) [22]. Produced thin films are polycrystalline or amorphous; however, the crystal quality can be further enhanced by annealing (Fig. 6d) [22]. 

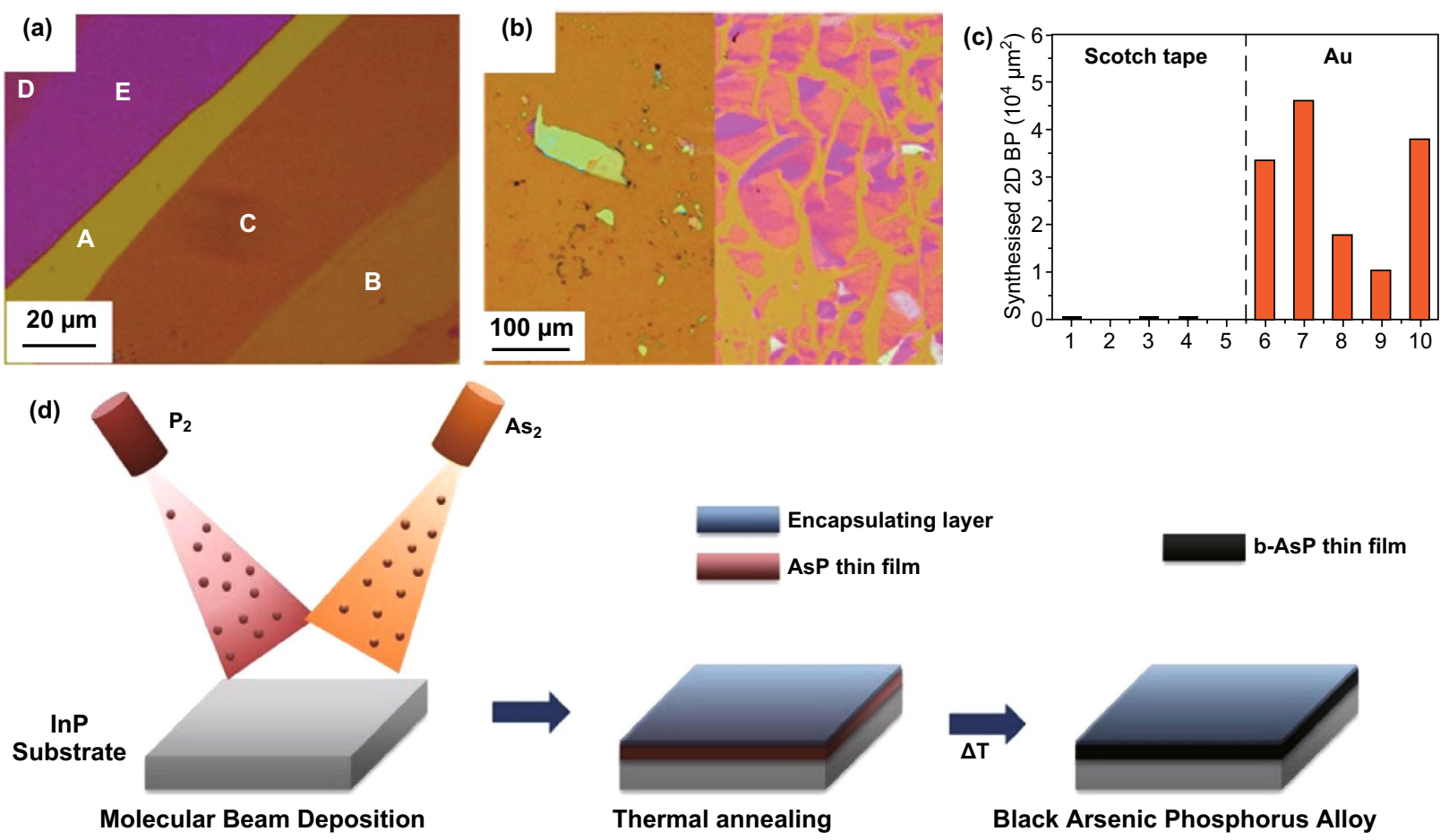

Fig. 6 Large-area synthesis of phosphorous compounds. a Optical microscope images of few layer black phosphorus (FLBPs) exfoliated via the Ag- assisted methods, b the left is FLBPs exfoliated using the normal "scotch-tape" method and the right is BP exfoliated using the Au-assisted method, and $\mathbf{c}$ the total area of FLBP on 10 different samples. Adapted with permission from Ref. [21]. Copyright 2018, Royal Society of Chemistry Publishing Group. d Schematic of wafer-scale MBE grown 2D b-AsP achieved by evaporation of $\mathrm{P}_{2}$ and $\mathrm{As}_{2}$ followed by thermal annealing. Adapted with permission from the Ref. [22]. Copyright 2018 American Chemical Society

\subsubsection{Xenes}

2D Xenes are the technologically significant emerging class of 2D materials in the design of fundamentally novel lowenergy nanoelectronics, spintronics and devices featuring room temperature quantum spin hall effects $[99,100]$. This class of materials offers versatile properties including semiconducting, superconducting, trivial and topological insulating phases. The materials including silicene, germanene, tellurene, borophene, stanene, bismuthene, plumbene, etc., are examples of the monoelemental crystals of silicon, germanium, tellurium, boron, tin, bismuth and lead, respectively. Only a few of these materials have been realised in 2D large lateral dimensions $(>100 \mu \mathrm{m})$ including silicene [39], germanene [101] and tellurene [40] development of large-scale synthesis strategies for others such as borophene [41], stanene [102] and plumbene [103], bismuthene [104] is ongoing.

Interestingly, large-area syntheses of 2D Xene materials are achieved using different methods which lack universality.
Silicene is synthesised using MBE on $\operatorname{Ag}(111) /$ mica substrates (Fig. 7a) [39]. Germanene layers have been synthesised in a three-stage synthesis. In the first stage, $\mathrm{Si}_{0.65} \mathrm{Ge}_{0.35}$ is epitaxially deposited. In the second and third stage, the film is immersed in $\mathrm{N}_{2}$ plasma and annealed, respectively, to produce atomically thin large layers of Germanene (Fig. 7b) [101]. Most of the growth methodologies rely on synthesis directly on substrates, and solution-based synthesis of largearea materials are rarely found. Wang et al. [40] developed $2 \mathrm{D}$ tellurene sheets in suspensions with a high yield of products featuring high mobility of up to $700 \mathrm{~cm}^{2} \mathrm{~V}^{-1} \mathrm{~s}^{-1}$ in room temperature (RT).

Borophene is emerging 2D sheet of boron suitable for applications in high performance and flexible optoelectronics [41, 42, 106, 107]. Wu et al. [41] synthesised 2D borophene crystals on $\mathrm{Cu}$ (111) with MBE method at ultra-high vacuum $\left(2 \times 10^{-10}\right.$ torr $)$ with a maximum achieved single crystal with areas of up to $100 \mu \mathrm{m}^{2}$. However, compared with other 2D Xenes, borophene has yet to achieve lateral dimensions exceeding tens of micrometres [42]. 

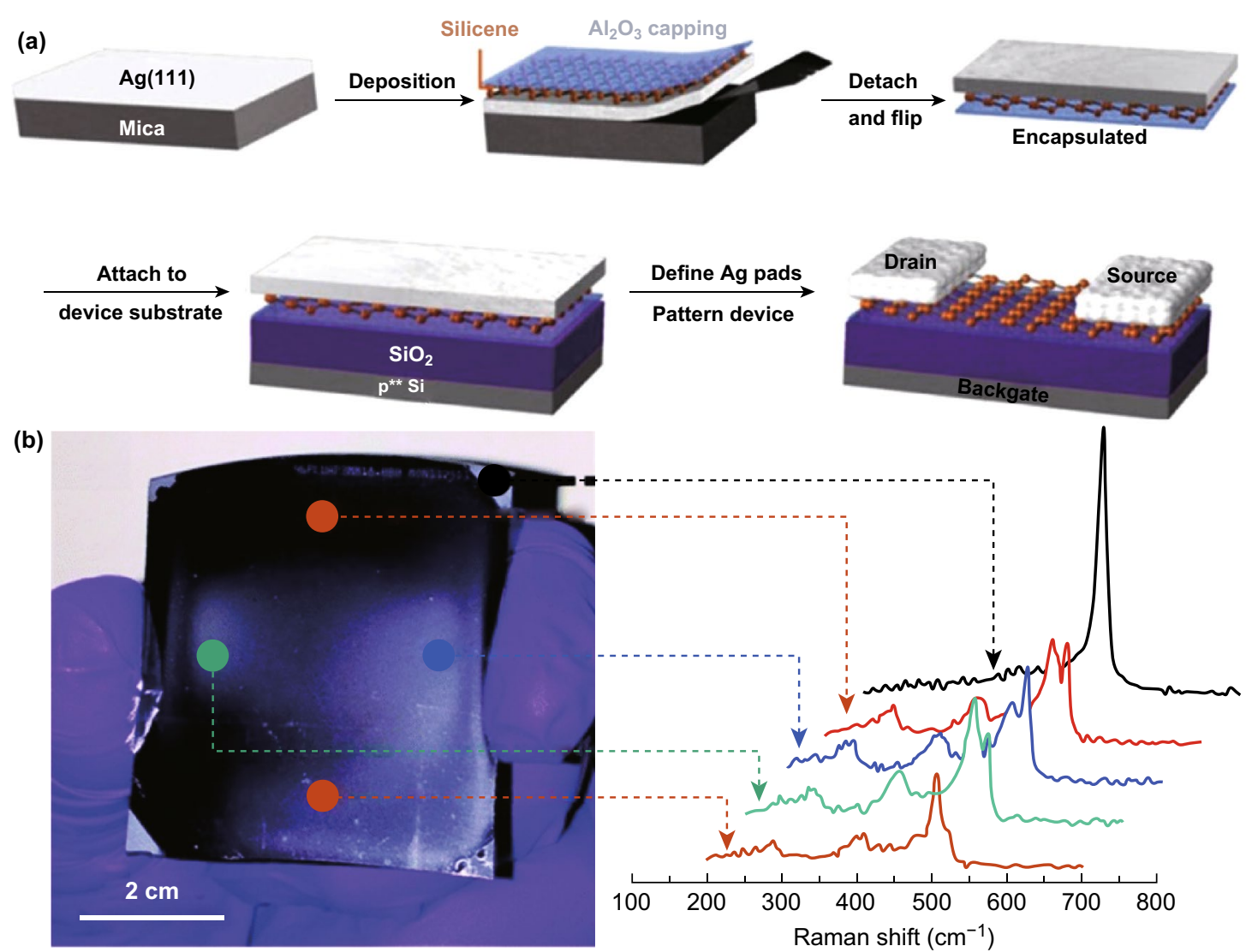

Fig. 7 Large-area synthesis of 2D Xenes. a Schematic representation of the synthetic steps for the synthesis of silicene including epitaxial deposition, $\mathrm{Al}_{2} \mathrm{O}_{3}$ capping, transfer onto a substrate and device fabrication. Adapted with permission from Ref. [105]. Copyright 2016, Elsevier B.V. b Large-area synthesised multi-layered germanene using $\mathrm{N}_{2}$ plasma assisted-process and corresponding Raman spectra at multiple locations. Adapted with permission from Ref. [101]. Copyright 2015, Royal Society of Chemistry Publishing Group

Bismuthene, stanene and plumbene have not been achieved in large areas; however, they can potentially be derived from their large-area 2D metallic sheets. For example, the synthesis of 2D bismuth layers in large areas is discussed in the next section; however, the referenced articles lack direction in achieving crystal structures that are similar to bismuthene. Further synthesis optimisation and substrate engineering are needed to achieve them as 2D Xenes crystals.

\subsubsection{Bismuth Compounds}

Bismuth is a post-transition metal which its compounds are increasingly gaining attention due to their topological insulating (TI) properties for future low-energy electronics device integration. Several methods for the synthesis of large-area bismuth compounds have been investigated entailing PLD, MBE, CVD and LM. PLD produces centimetre scale, Bi Sheets, with relatively good crystal quality and high mobility of $220 \mathrm{~cm}^{2} \mathrm{~V}^{-1} \mathrm{~s}^{-1}$ [108] (Fig. 8a). This may potentially provide pathways to the synthesis of bismuthene layers. MBE methods are widely adopted growth methods of bismuth selenides and tellurides with the large-area coverages for the study of TI behaviour [43, 44]. However, MBE is expensive to operate, difficult to integrate to industry and results in several $\mathrm{X}-\mathrm{Bi}-\mathrm{X}-\mathrm{Bi}-\mathrm{X}$ $(\mathrm{X}=\mathrm{Te}$ and $\mathrm{Se})$ quintuple layers $(\mathrm{QL})$ with relatively small domains $[43,44,110]$. Ultra-high vacuum condition enables an in situ analysis of these materials and to protect against n-type doping if exposure to air which is an advantage of MBE over CVD methods [111]. Extensive research is still underway using $\mathrm{MBE}$ to achieve high-quality TI crystals including $\mathrm{Bi}_{2} \mathrm{Te}_{3}$ and $\mathrm{Bi}_{2} \mathrm{Se}_{3}$ which are the material of choice for the study of magneto-transport properties 

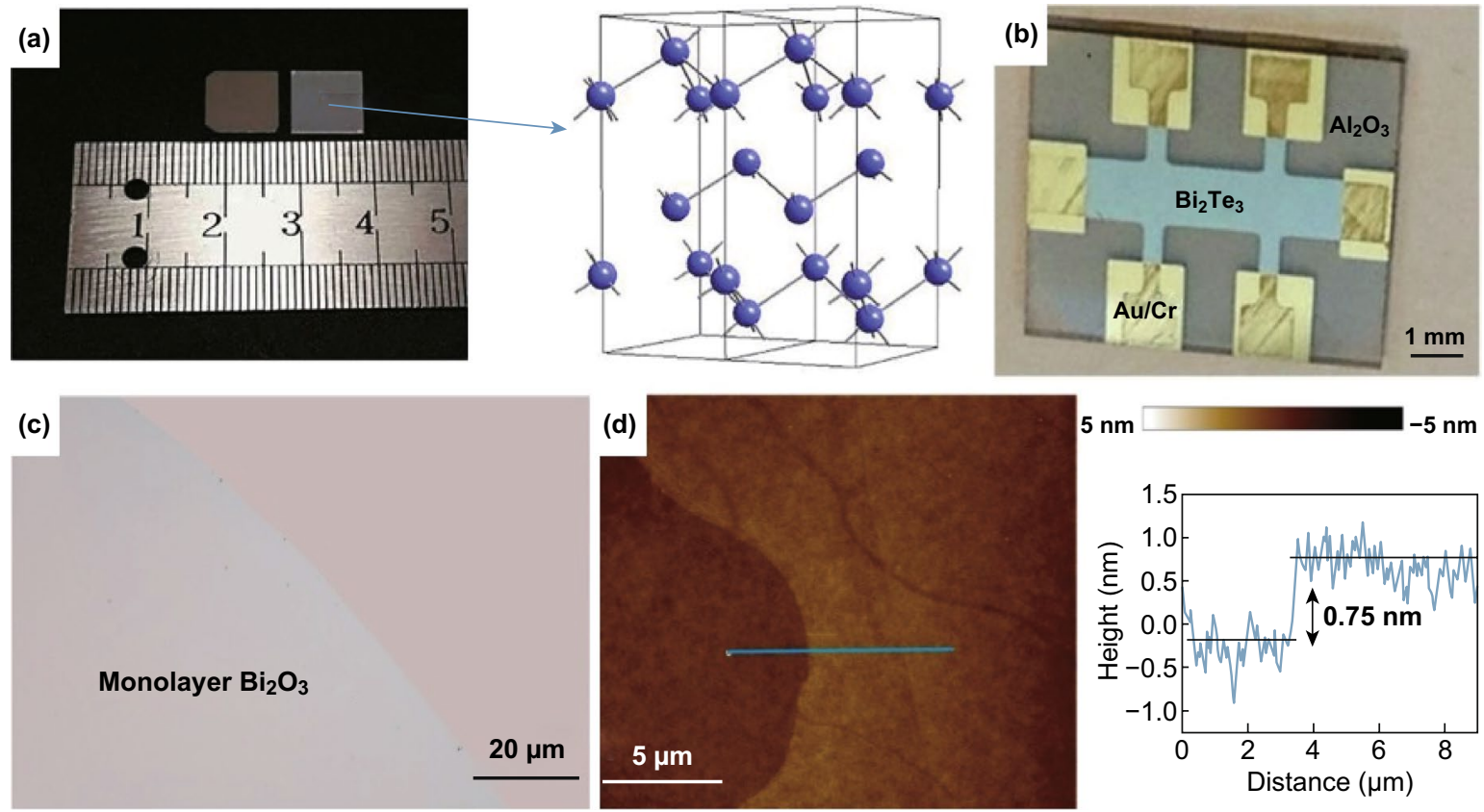

Fig. 8 Large-area synthesis of bismuth compounds. a PLD growth of $2 \mathrm{D}$ bismuth on $\mathrm{SiO}_{2}$ (left) and $\mathrm{Al}_{2} \mathrm{O}_{3}$ (right) and the crystal structure elucidating bulk bismuth. Adapted with permission from Ref. [108]. Copyright 2019, Wiley. b Optical image of a hall bar device from MBE grown $\mathrm{Bi}_{2} \mathrm{Te}_{3}$ on $\mathrm{Al}_{2} \mathrm{O}_{3}$ substrate featuring millimetre long topological insulator properties. Adapted with permission from Ref. [109]. Copyright 2017, Elsevier B.V. c LM printed $\mathrm{Bi}_{2} \mathrm{O}_{3}$ from the surface of molten bismuth in an oxygen controlled environment and $\mathbf{d}$ AFM showing a thickness profile of a monolayer. Adapted with permission from Ref. [15]. Copyright 2018, Royal Society of Chemistry Publishing Group

due to strong spin-orbit coupling (Fig. 8b) [109]. However, several critical 2D compounds of Bi including chalcogenides have not been realised with lateral sizes larger than $100 \mu \mathrm{m}$ by CVD methods [112]. Sub-millimetre single crystals of $\mathrm{Bi}_{2} \mathrm{O}_{2} \mathrm{Se}$ have been synthesised by low-pressure CVD (LPCVD) with ultra-high mobility of $29,000 \mathrm{~cm}^{2} \mathrm{~V}^{-1} \mathrm{~s}^{-1}$ at $1.9 \mathrm{~K}$ and $450 \mathrm{~cm}^{2} \mathrm{~V}^{-1} \mathrm{~s}^{-1}$ in RT [45]. Space-confined CVD method using stacked mica substrates for growth of BiOI with more than $100 \mu \mathrm{m}$ grain sizes is synthesised [46]. Space confinement is an effective method to obtain uniform thicknesses of 2D sheets during the CVD growth. In a space-confined environment, a narrow gap is created for reactants to reduce and control the nucleation density and growth rates [113]. Choosing a substrate can also enhance more homogenous nucleation rates such as atomically flat mica with no dangling bond to make $\mathrm{BiOI}$ [46]. 2D $\mathrm{Bi}_{2} \mathrm{O}_{2} \mathrm{Se}$ with high stability in air and high-motility semiconducting are grown on mica at LPCVD using $\mathrm{Bi}_{2} \mathrm{O}_{3}$ powder and $\mathrm{Bi}_{2} \mathrm{Se}_{3}$ bulk precursors with large domain sizes and ultra-high mobility. Messaela et al. [15] synthesised monolayer of bismuth oxide with sub-nanometre thicknesses using LM-based exfoliation
(Fig. 8c, d). Molten Bi surfaces developed a highly crystalline with large lateral dimension and thinnest reported layers of $\alpha-\mathrm{Bi}_{2} \mathrm{O}_{3}[15]$.

Considering Moore's law approaching its limits, emerging materials provide avenues to overcome current technological challenges and limitations. Several new materials have emerged, providing avenues for the exploration of novel heterostructures and next-generation electronics and optoelectronics devices. Many of the emerging 2D materials yet to be realised in large areas exceeding $100 \mu \mathrm{m}$ lateral dimensions including borophene, stanene, plumbene and bismuthene. A method to achieve these monoelemental structures can be through reduction reactions which should be attempted $[114,115]$.

\subsection{Non-layered Materials}

Atomically thin 2D materials with non-layered structures possess exciting properties. Significant advances in the development of non-layered ultrathin 2D materials such as noble metals, metal oxides and metal chalcogenides have 
been seen in recent years. Due to the hardship of strong in-plane bonds breaking (e.g. covalent, metallic and ionic bonding) and the lack of intrinsic anisotropic growth driving force, it is still a great challenge to synthesise ultrathin 2D nanosheets with non-layered structures. In this point of view, a bottom-up technique such as wet chemical synthesis, ionic layer epitaxy (ILE), liquid metal-based exfoliation, CVD, PVD, sputtering and templated synthetic strategy has been successfully developed and continuously optimised to break the thermodynamic equilibrium state and control the aggregation kinetics, which consequently leads to the anisotropic growth of atomically thin non-layered nanocrystals [116-118]. However, large area, high-quality and homogeneous production of non-layered $2 \mathrm{D}$ sheets has proven to be a key challenge. Only very few numbers of articles have addressed such a challenge so far. Indium tin oxides (ITO) which is an important class of 2D transparent conductive oxides have been synthesised in 2D and large scale using a simple sputtering method [47]. Wang et al. proposed the wafer-scale growth of $\mathrm{CoO}$ nanosheets and large-area $\mathrm{ZnO}$ nanosheets using adaptive ionic layer epitaxy (AILE) method. In AILE, at a two-phase interface (basically a water-air), an ionic amphiphilic molecular monolayer is engaged, and crystals grow at the interface absorbed by electrostatic and covalent interactions between the precursor ions and the functional groups on the amphiphilic molecules (Fig. 9i-iv) [48]. Initially, tiny nanocrystals are generated and self-organised stochastically into a continuous amorphous film (Fig. 10vi). These nanocrystals then attach to each other through the interatomic bonds between high energy facets at an aligned orientation (Fig. 10vii-viii). Finally, the amorphous film is fully crystallised, and a single-crystal nanosheet is hence generated (Figs. 10ix) [49]. However, a small number of nanoparticles (Figs. 9v, x, 10i) were sparsely distributed on top of the nanosheet due to the transfer and drying process. Additionally, such a process limits to a few types of nanomaterials and cannot be readily extended to others due to the rigorous synthetic conditions, such as concentrations of reactants, surfactant selection and reaction temperature and time [116]. This method also led to a large area of defects as observed from the TEM image in Fig. 10v.

Alsaif et al. synthesised large-area $2 \mathrm{D} \mathrm{SnO} / \mathrm{In}_{2} \mathrm{O}_{3}$ heterostructures by touching the surface oxide layers from the liquid tin and indium onto the substrate separately [16]. LM synthesis is also shown to produce centimetre-scale gallium oxide $\left(\mathrm{Ga}_{2} \mathrm{O}_{3}\right)$ that can be isolated from the liquid $\mathrm{Ga}$ surface [50, 51]. Metal inclusions were observed on $\mathrm{Ga}_{2} \mathrm{O}_{3}$ nanosheet, which was removed by a simple mechanical ethanol washing method (Fig. 11). During the cleaning procedure, a beaker of ethanol was heated to $78^{\circ} \mathrm{C}$. The $\mathrm{SiO}_{2} / \mathrm{Si}$ wafer with an exfoliated $2 \mathrm{D} \mathrm{Ga}_{2} \mathrm{O}_{3}$ sheet was then plunged in the hot ethanol and gently wiped out the metal inclusions with the help a wiping tool (cotton bud). Exfoliated non-layered $\mathrm{Ga}_{2} \mathrm{O}_{3}$ was converted to $\mathrm{GaPO}_{4}$ utilising a simple CVD process at low temperatures $\left(300-350{ }^{\circ} \mathrm{C}\right)$. The 2D nanosheets were uniform, continuous and thermally stable up to $600{ }^{\circ} \mathrm{C}$ [50]. Using similar LM synthesis strategy, Syed et al. [51] also successfully synthesised atomically thin wafer-scale gallium nitride $(\mathrm{GaN})$ with a thickness
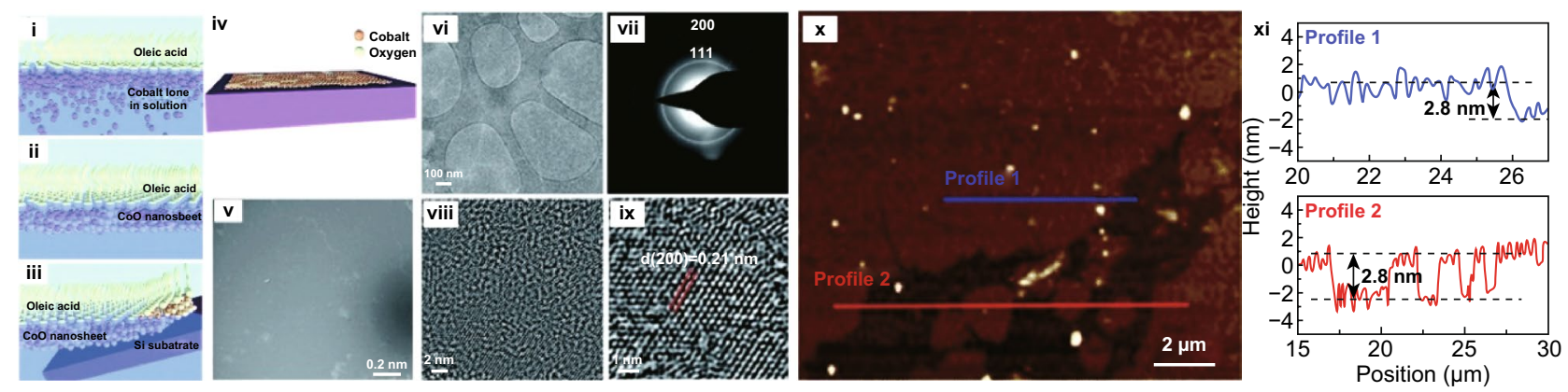

Fig. 9 Large-area synthesis of 2D CoO. Schematic illustration of the processing and formation of $\mathrm{CoO}$ nanosheets at the water-air interface (i-iv). Co ions crystalise into macroscopic, continuous nanocrystalline CoO nanosheets as large as the water-air interface. (v) SEM image covering a Si substrate surface, (vi) TEM image, (vii) corresponding SAED pattern of a CoO nanosheet. (viii, ix) HRTEM images of CoO polycrystalline nature with an average grain size $\sim 3 \mathrm{~nm}$ and fully crystallised structure with grain and grain boundaries. (ix, x) Typical AFM image and corresponding height profile along the blue and red lines in (ix) showing a minimal roughness factor of $0.39 \mathrm{~nm}$ and a uniform film thickness of $2.8 \mathrm{~nm}$ of $\mathrm{CoO}$ nanosheet. Adapted with permission from Ref. [48]. Copyright 2017, Royal Society of Chemistry Publishing Group. (Color figure online) 

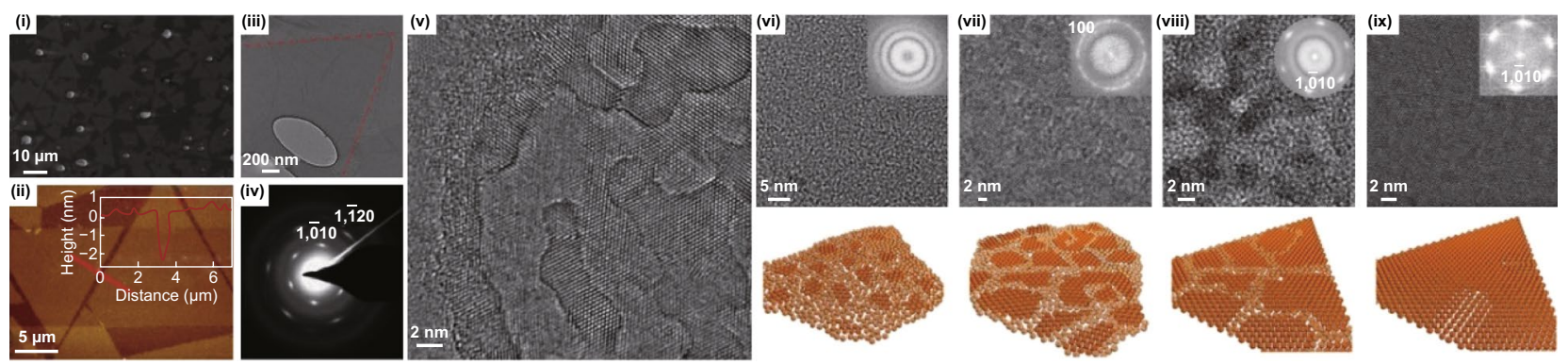

Fig. 10 Large-area synthesis of 2D ZnO. (i) SEM image, (ii) AFM height profile, (iii) TEM, (iv) corresponding SAED pattern is shown in iii, (v) HRTEM image of $\mathrm{ZnO}$ nanosheet showing overlayer growth. (vi-ix) TEM images and graphic illustrations are showing the time-dependent evolution of $\mathrm{ZnO}$ nanosheets. Fast Fourier transform (FFT) patterns of the TEM images are at the insets, respectively. The amorphous area was entirely crystallised, and the nanosheet became single-crystalline over different reaction time. Adapted with permission from Ref. [49]. Copyright 2016, Nature Publishing Group

(i)

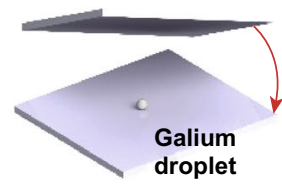

(ii)

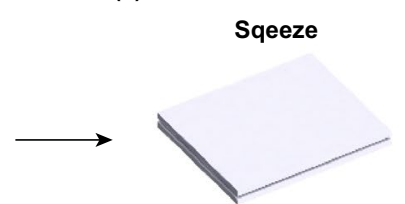

(iii)

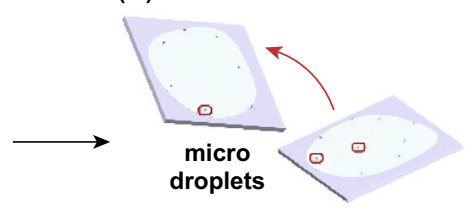

(iv)

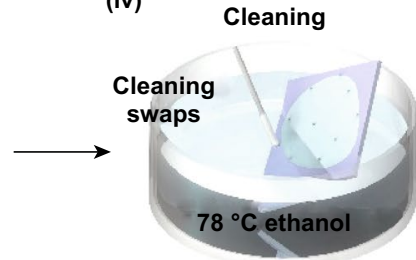

(v)
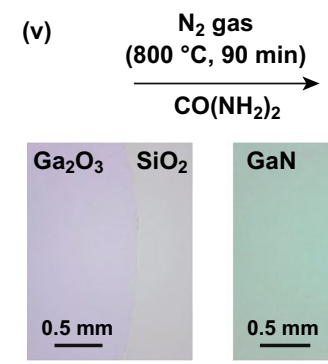
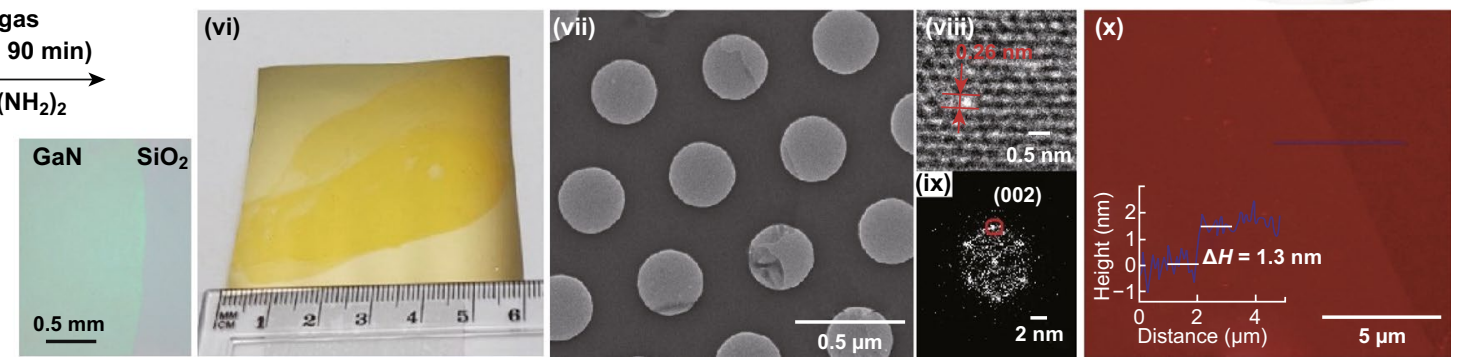

Fig. 11 Large-area synthesis of $2 \mathrm{D} \mathrm{GaN}$. (i-v) Schematic illustration of the synthesis and cleaning process of $2 \mathrm{D} \mathrm{Ga} \mathrm{Ga}_{3}$ on $300 \mathrm{~nm} \mathrm{SiO} / 2 \mathrm{Si}$, then transferring them to the $2 \mathrm{D} \mathrm{GaN}$ nanosheet from using ammonolysis. (vi) Optical image of $\mathrm{LM}$ synthesised $\mathrm{Ga}_{2} \mathrm{O}_{3}$ on $\mathrm{SiO}_{2} / \mathrm{Si}$ wafer in centimetre scale. (vii) TEM micrograph, (viii) HRTM lattice fringes, (ix) the corresponding FFT pattern, and (x) Typical AFM topography with height profile along the blue line of the GaN film. Adapted with permission from Ref. [51]. Copyright 2019, American Chemical Society Publishing Group. (Color figure online)

of $1.3 \mathrm{~nm}$ and indium nitride ( $\mathrm{InN}$ ) with the thickness of $2 \mathrm{~nm}$. In this article, isolated $\mathrm{Ga}_{2} \mathrm{O}_{3}$ sheets were converted into $\mathrm{GaN}$ using a high-temperature ammonolysis reaction at $800{ }^{\circ} \mathrm{C}$, where urea was used as an ammonia precursor (Fig. 11). More recently, LM synthesis methods were used to produce another non-layered compound $2 \mathrm{D} \mathrm{Ga}_{2} \mathrm{~S}_{3}[52]$. It is also demonstrated that liquid metals can act as a reaction solvent and dissolve other metallic elements. In the air, the surface of liquid metals forms an ultrathin oxide layer with the composition that is dominated by the metal oxide with more favourable energy of the reaction. Using this phenomenon, Zavabeti et al. [14] transferred large-area surface oxides of several metals, including $\mathrm{Gd}_{2} \mathrm{O}_{3}, \mathrm{Al}_{2} \mathrm{O}_{3}$ and $\mathrm{HfO}_{2}$ by vdW touch transfer exfoliation. The liquid metal frameworks, however, are suffered from low solubility of other metallic elements such as Mo and W. In addition, several other elements are energetically not favourable to achieve. Another state-of-the-art method to produce 2D nanosheet suspensions has been pioneered by Sasaki group to provide $2 \mathrm{D}$ oxide sheets of titanium, manganese and niobium (Fig. 12) [72, 119]. Ma et al. [72] extended the protocols to achieve several other 2D elemental hydroxides.

Template-based synthesis methods have been widely used for the growth of anisotropic nanocrystals in which the 
crystal growth can be confined in a specific dimension [120, 121]. A continuous and uniform amorphous basic aluminium sulphate (BAS) layer was first coated on the graphene oxide (GO) surface through a homogeneous deposition method. After that, GO was removed from the composite, and the BAS layer was converted into $\mathrm{Al}_{2} \mathrm{O}_{3}$ nanosheet by calcination at $800{ }^{\circ} \mathrm{C}$. The precipitation is a slow process and usually, takes several hours to precipitate (BAS) all the aluminium ions. Such a slow reaction rate allows fine-control of the thickness of the deposited BAS layer on the GO sheets. Recently, Li et al. [122] reported the growth of largearea $2 \mathrm{D}$ transition metal phosphides (TMPs) $\left(\mathrm{Co}_{2} \mathrm{P}, \mathrm{MoP}_{2}\right.$, $\mathrm{Ni}_{12} \mathrm{P}_{5}$ and $\mathrm{WP}_{2}$ ) with the aid of water-soluble salt crystals as growth templates (Fig. 13i-iv). The 2D TMPs showed well-defined exposed crystal facets, such as the (130) facet for $\mathrm{Co}_{2} \mathrm{P}$, the (010) facet for $\mathrm{MoP}_{2}$, the (010) facet for $\mathrm{Ni}_{12} \mathrm{P}_{5}$ and the (001) facet for $\mathrm{WP}_{2}$. The area of $2 \mathrm{D}$ morphology is over $50 \mu \mathrm{m}^{2}$ with a thickness of 4, 2, 5, 1.8 and $2.3 \mathrm{~nm}$ for $\mathrm{Co}_{2} \mathrm{P}, \mathrm{MoP}_{2}, \mathrm{Ni}_{12} \mathrm{P}_{5}$ and $\mathrm{WP}_{2}$, respectively. It was suggested that both the salt crystal geometry and lattice matching could guide and promote the lateral growth of 2D TMPs, while the thickness could be well-balanced by the raw material supply [15]. However, this technique did not afford smooth and compact 2D nanosheets. Additionally, well matching of lattice planes between target 2D nanosheets and template is the critical requirement for the formation of $2 \mathrm{D}$ anisotropic nanosheets.

Another typical method that has been extensively used for the synthesis of non-layered 2D materials is hydrothermal synthesis. The large-scale $\mathrm{Co}_{3} \mathrm{O}_{4}$ nanosheets with a thickness of less than $3 \mathrm{~nm}$ have been prepared by a nonsurfactant and substrate-free hydrothermal method into a homogeneous reactor with the subsequent thermal annealing treatment [123]. In this method, cobalt ammonia complexes reconstruct under a high concentration of ammonia during hydrothermal conditions which were used to fabricate $2 \mathrm{D} \mathrm{Co}_{3} \mathrm{O}_{4}$ nanosheets. The area and thickness of $\mathrm{Co}_{3} \mathrm{O}_{4}$ are up to $30 \mu \mathrm{m}^{2}$ and $2.9 \mathrm{~nm}$, respectively. Feng et al. [123] explored that hydrothermal temperature and hydrothermal time have significant impacts on the morphology and yield. In this process, $140{ }^{\circ} \mathrm{C}$ is the optimum temperature to form high-quality $2 \mathrm{D}$ sheets. At lower temperatures, residues of reaction byproducts remained in the interlayers of the 2D nanosheets. On the other hand, at higher temperatures, ammonia becomes ionised; hence, dissociative ammonia is impotent in the $2 \mathrm{D}$ nanosheet formation [123].

Non-layered crystals incorporate an abundant library of materials which require more investigation to enable achieving them in stratified large-area 2D morphologies. Novel synthetic methodologies include liquid metals [14] and soft chemical processes [72, 119]. For liquid metal synthesis, gallium as a solvent should be substituted with another metal with less energy of reaction and as well as providing high-entropy liquid metal alloys with higher loading of added reactants. The reactive gas and solvents surrounding liquid metal alloys can also be modified to offer other compositions than oxides. The soft chemical processes developed by Sasaki group can also be possibly applied to a more variety of elements to achieve 2D layered oxides that are otherwise challenging to obtain [124]. (a)

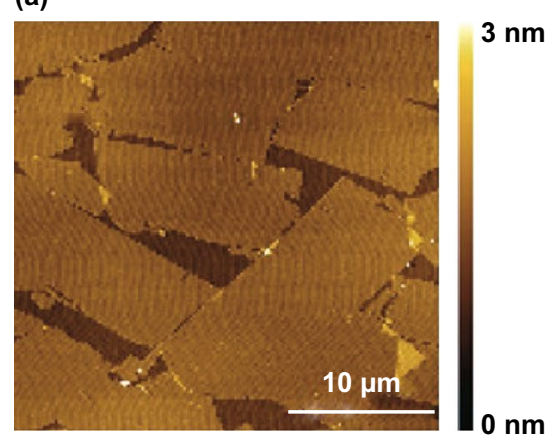

Titanium oxide (b)

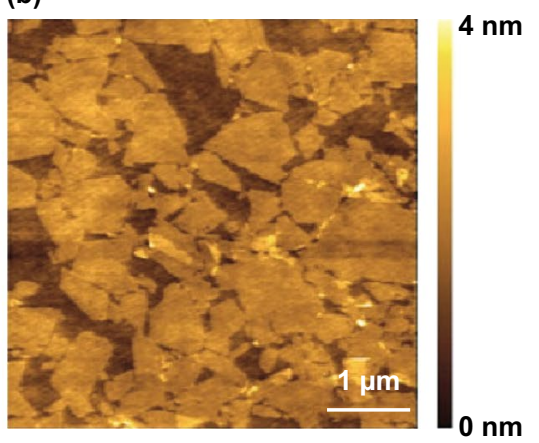

Manganese oxide (c)

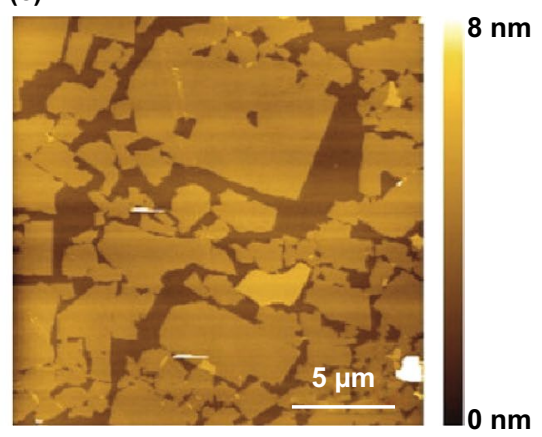

Niobium oxide

Fig. 12 AFM images of achieved 2D oxides from the soft chemical process. a Titanium oxide, b Manganese oxide, c Niobium oxide. Adapted with permission from Ref. [72]. Copyright 2015, American Chemical Society Publishing Group 


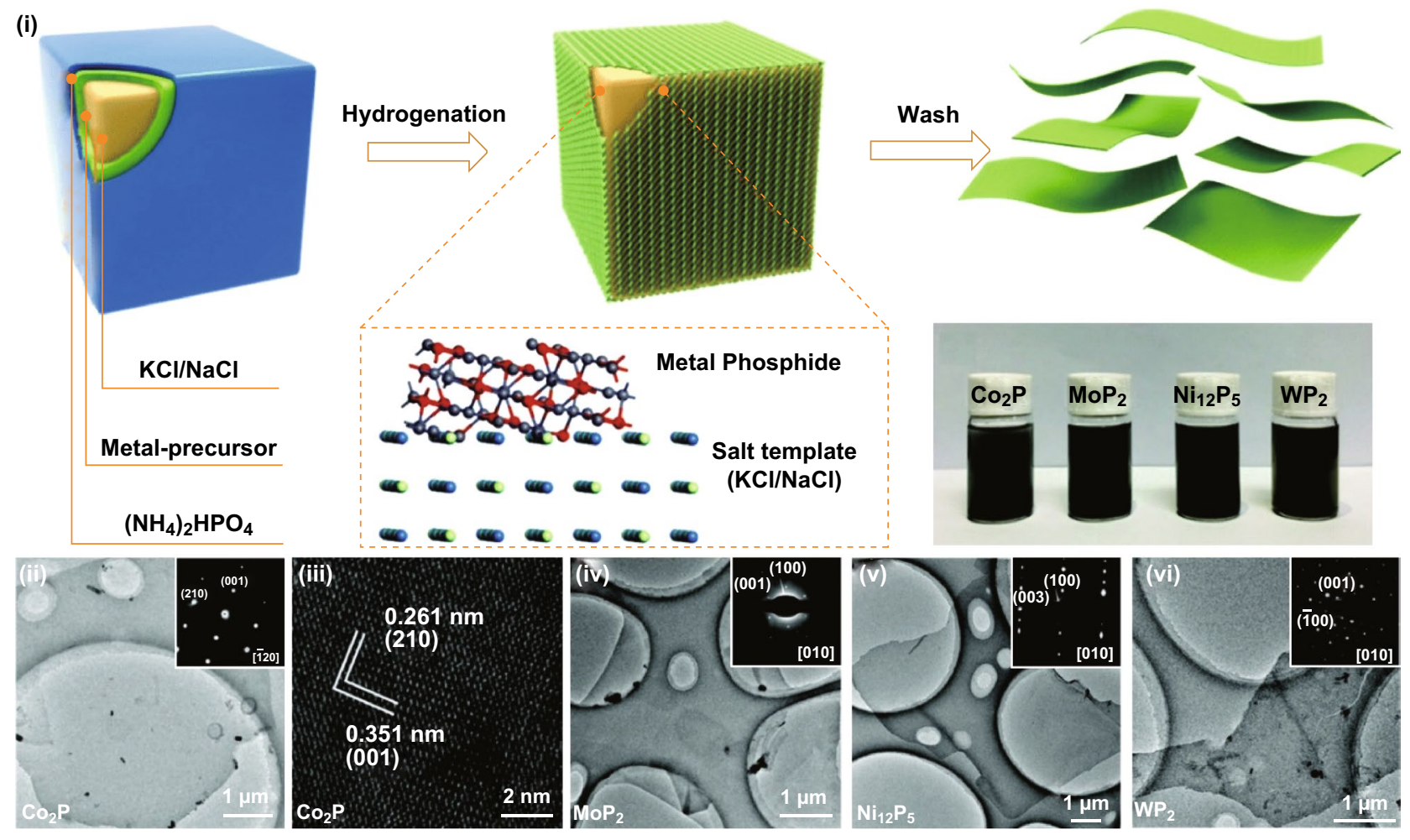

Fig. 13 Large-area synthesis of 2D metal phosphides. (i) Schematic representation of the synthesis process and optical images of 2D metal phosphides. (ii) TEM (inset: the corresponding SAED pattern) and (iii) HRTEM images of $2 \mathrm{D} \mathrm{Co}_{2} \mathrm{P}$. TEM images (inset: the corresponding SAED pattern) of $2 \mathrm{D} \mathrm{MoP} 2$ (iv), $\mathrm{Ni}_{12} \mathrm{P}_{5}(\mathbf{v})$ and $\mathrm{WP}_{2}$ (vi). Adapted with permission from Ref. [122]. Copyright 2018, The Royal Society of Chemistry Publishing Group

\subsection{Graphene}

Graphene as the first isolated 2D material provides an extensive account of synthesis optimisation. Lack of bandgap in graphene has limited its use in logic devices and the successful integration into large-area novel electronic and optoelectronic devices. Therefore, scientists have either engineered graphene to induce a bandgap or used it in heterostructures $[125,126]$. This review will only summarise large-area graphene synthesis, providing valuable insight that may be applied to the synthesis of other semiconducting 2D materials. Similar to the synthesis approaches of other 2D materials, CVD holds promise for large-scale production of high-quality single crystals of graphene with uniform thickness. Metallic surfaces are found to be one of the appropriate substrates to realise large-area growth [53, 127]. Vlassiouk et al. [128] exploited the evolutionary selection approach in the Czochralski process to obtain foot-long single-crystal quality graphene on $\mathrm{Cu}-\mathrm{Ni}$ alloy surfaces [127]. In this method, the fastest growing domain orientation dominates the crystal facet direction with growth rates as high as $2.5 \mathrm{~cm} \mathrm{~h}^{-1}$ [127]. Xu et al. [11] synthesised metre-sized graphene single crystals on $\mathrm{Cu}$ (111). Since $\mathrm{Cu}$ (111) has the same rotational symmetry of $\mathrm{C} 3$ as graphene with only $4 \%$ lattice mismatch, it provides a suitable surface for the growth of large-area single crystals [11]. However, most of the industrial $\mathrm{Cu}$ foils feature polycrystalline, and additional thermal annealing is needed to increase the $\mathrm{Cu}$ (111) facet size (Fig. 14a-d) [11]. Liquid metal melts can be used as an effective substrate for the synthesis of largearea CVD grown 2D materials with minimum imperfections [35, 76]. Similarly, molten copper foil is used as a substrate for the large-area synthesis of graphene with less grain boundary formation [129]. Interestingly, during the synthesis, highly aligned 2D graphene domains are produced in the direction of the gas flow (Fig. 14e-i) [129]. Sun et al. improved the synthesis growth rates up to four times. They reduced the synthesis temperature using carbon feedstock substitute precursors rather than methane, hence producing millimetre-sized single-crystal graphene [130]. Apart from 

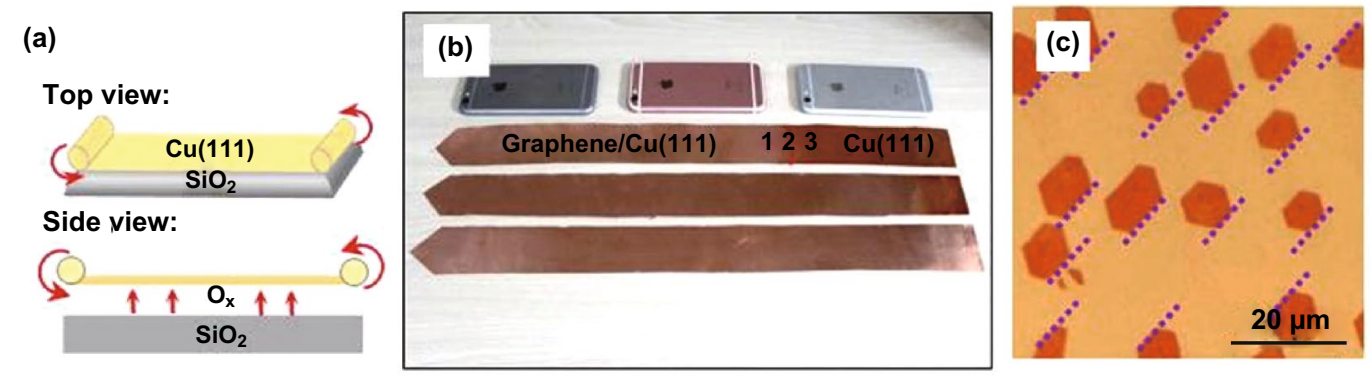

(d)
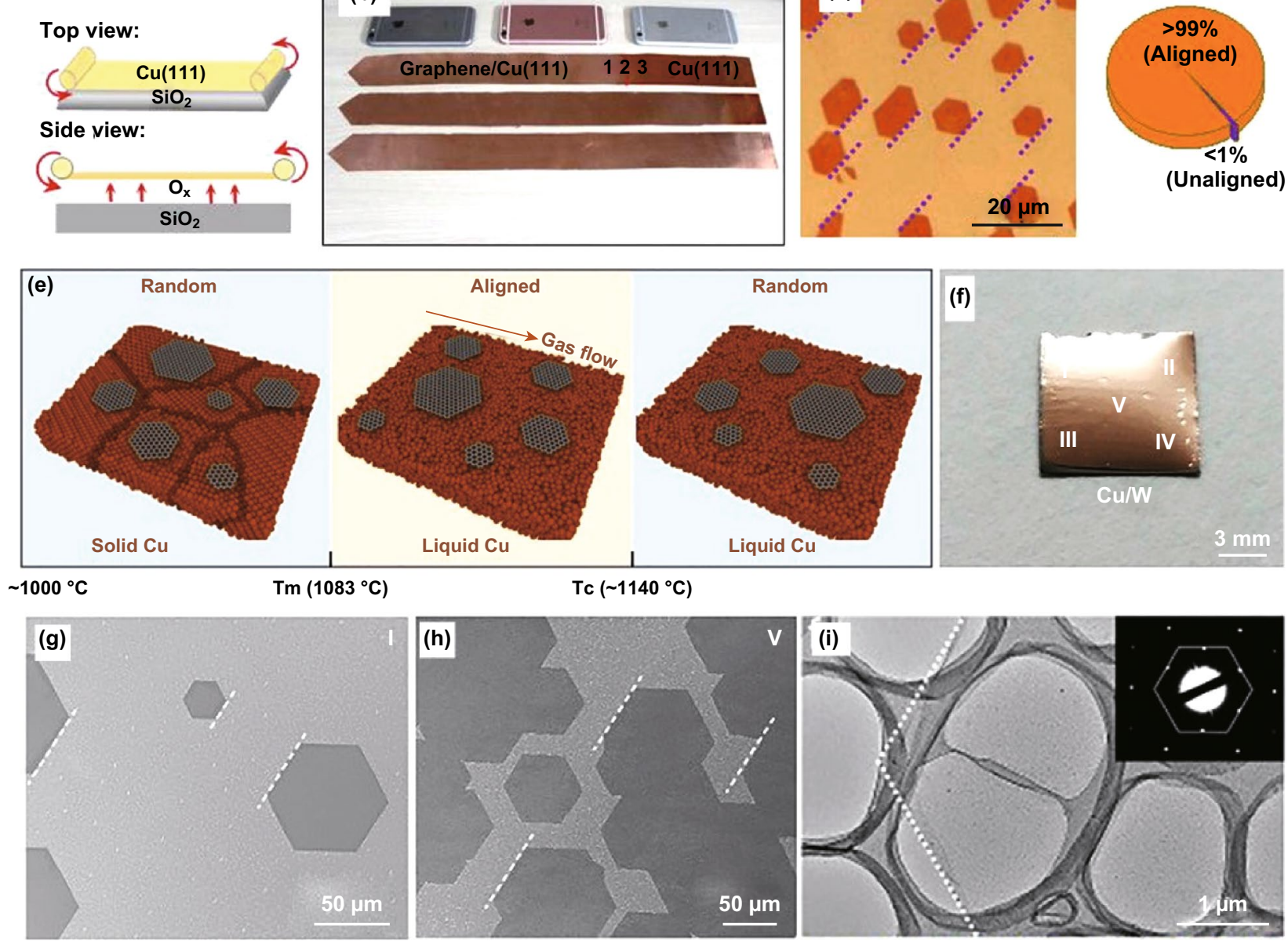

Fig. 14 Synthesis of large-area graphene. a Top and side schematic views of the continuous graphene film growth system, where the Cu (111) foil was placed above a $\mathrm{SiO}_{2}$ substrate with a small separation, for ultrafast growth. b $\mathrm{Cu}$ (111) foils with graphene coverages of $60 \%$ (top), $90 \%$ (middle), and 100\% (bottom), where the "shining" parts are graphene/Cu (left side). The three mobile phones are placed nearby as a reference for size. $\mathrm{c}$ Optical image of the arbitrarily distributed holes formed by $\mathrm{H}_{2}$ etching of the graphene film. Edges of the holes marked by the dashed lines are parallel with each other. d The proportion of the aligned graphene islands restrained from optical images. Adapted with permission from Ref. [11]. Copyright, 2017, Elsevier. e Schematic illustration of graphene formation behaviour under different temperatures. f Photograph of a $1 \times 1 \mathrm{~cm}^{2}$ sample after graphene growth. $\mathbf{g}$, h SEM images of graphene parts in different areas. $\mathbf{i}$ TEM image revealing high-quality singlecrystal monolayer of graphene. Adapted with permission from Ref. [129]. Copyright 2019 American Chemical Society

CVD, large-area graphene has been made using PLD [131], laser irradiation methods [131] and enhanced ME (Fig. 15) [20]. Enhanced ME method provided large-area monolayers of graphene and $\mathrm{Bi}_{2} \mathrm{Sr}_{2} \mathrm{CaCu}_{2} \mathrm{O}_{\mathrm{x}}$ (BSCCO) monolayers. In this method, the surface was treated with plasma, and the sticky tape was left at elevated temperature to enhance the sticktion and consequently, vdW exfoliation. Several reliable transfer methods are used for transferring a largearea 2D graphene enabling device integration [132-134]. Shivayogimath et al. used laminator and polyvinyl alcohol polymer foil to transfer large-area graphene from $\mathrm{Cu}$ foil. Authors extended the method to transfer multilayer hBN from $\mathrm{Cu}$ and $\mathrm{Fe}$ foils [132]. Wang et al. [133] introduced a novel strategy to use the wetting-induced transfer of graphene sheets from solvent interfaces. Karmakar et al. [134] transferred centimetre-scale graphene sheets from $\mathrm{Cu}$ foil to $\mathrm{SiO}_{2} / \mathrm{Si}$ substrates using the copolymer-assisted technique. Roll to roll transfer of large-area patterned graphene was demonstrated by Choi et al. [135] as a promising method for commercially viable transfer technique to flexible substrates. Graphene and its derivatives, for example, GO, reduced graphene oxide (rGO) and functional graphene oxide (fGO) have been investigated for integration into functional devices. Nevertheless, they are also used as a template for 

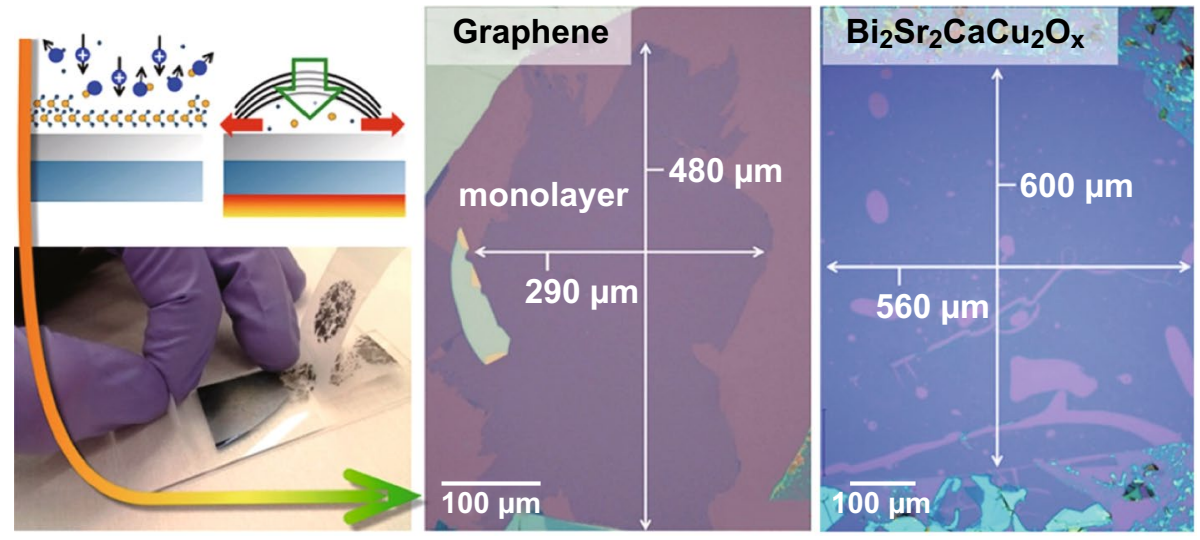

Fig. 15 ME isolation of graphene. Schematic illustration of a modified ME route and optical images of the isolation of the large-area graphene and BSCCO monolayers using the same technique, respectively. In this technique, the $\mathrm{SiO}_{2} / \mathrm{Si}$ surface was cleaned with $\mathrm{O}_{2}$ plasma, followed by annealing and peel-off. Adapted with permission from Ref. [20]. Copyright 2015, American Chemical Society

large area producing other 2D materials $[54,136]$. GO has been recognised as a common template for synthesis of $2 \mathrm{D}$ materials, as it holds a large amount of oxygen-containing functional groups and shows strong affinity towards the inorganic materials [120, 136]. Also, it is highly dispersible in the solvent, which could direct the growth of high-quality ultrathin nanosheets. Huang et al. demonstrated the synthesis of ultrathin $2 \mathrm{D} \mathrm{Al}_{2} \mathrm{O}_{3}$ nanosheets with the thickness of $\sim 4 \mathrm{~nm}$ and size $>10 \mu \mathrm{m}$ by duplicating the shape of GO [136].

Graphene as a popular 2D material currently holds the record in achieved lateral sizes of the single crystal [11]. Several of the synthetic methods should be employed to achieve semiconducting 2D materials as well as using the large-area synthesised graphene and its derivatives as a template for producing other large-area single crystals.

\section{Defect Formations and Crystal Quality}

The periodic arrangement of atoms in crystal structures may not occur in a perfect regular lattice due to the presence of defects. Variety of low-dimensional defects exist in 2D materials that are summarised as: (I) zero-dimensional (0D) point defects including vacancies, antisites, substitutional impurities and adatoms. (II) One-dimensional defects (1D) include grain boundaries, twin boundary, edges and dislocations. (III) 2D defects, including holes, scrolls, wrinkles and folds [137].
These low-dimensional defects substantially influence device performances. Single crystals or crystal with a low density of defects are usually defined as high quality. However, defects provide an additional feature to effectively engineer some of the optical and electronic properties of 2D materials. Therefore, tremendous efforts have been devoted to controlling the defect formation during the synthesis of 2D materials [138].

\subsection{Defects Formation and Engineering During the Synthesis}

ME 2D materials from high-quality crystals feature intrinsic point defects with less controllability on the defects generation [139]. MBE offers precise control over morphology and is shown by Loh et al. [140] to be an effective method to control the stoichiometry of niobium selenide by controlling flux ratio and substrate temperature during growth on $\mathrm{Au}$ (111) substrate. For the chemical growth processes, several structural defects are inherently created according to the thermodynamic conditions of the related synthetic strategies [141]. CVD provides highly crystalline 2D TMDs but with inherent defects. CVD is a relatively fast technique to synthesis large-area 2D materials, and the thermodynamic conditions can be altered for the controlled generation of these defects. For example, intrinsic 0D point defects in the crystal structure of TMDs during CVD and thermal reduction/sulphurisation growth are elucidated in Fig. 16a-c [141-143]. Zhang et al. and Yu et al. demonstrated changing 
in the thermodynamic condition during the CVD synthesis of $\mathrm{WS}_{2}$ to control structural defects [138, 144]. Lauhon et al. varied the growth condition (temperature of sulphur and exposure time) during the conversion of $\mathrm{MoO}_{3}$ to $\mathrm{MoS}_{2}$ to modify the stoichiometry during CVD [145]. To achieve defects growth, conversion from transition metal oxide to chalcogenides is the preferred method since the degree of chalcogenisation can be controlled more effectively [145]. The substrate has a profound effect on the quality of the CVD grown 2D TMDCs [146]; as shown by van der Zande et al. [146], preconditioning of substrate can increase the size and crystal quality of the synthesised $\mathrm{MoS}_{2}$. As a result, $\mathrm{MoS}_{2}$ with large size grains of up to $120 \mu \mathrm{m}$ is synthesised, and defects at the mirrored twin boundaries are characterised as a periodic line of 8-4-4 ring defects (Fig. 17a) [146].

Leong et al. demonstrated the importance of precursor reactant rations in the development of $0 \mathrm{D}$ defects during the CVD synthesis of $\mathrm{MoS}_{2}$ [149]. For this synthesis, reagents' molarity ratios were varied and as a result, providing different stoichiometry of $\mathrm{MoO}_{\mathrm{x}} \mathrm{S}_{2-\mathrm{x}}$. This strategy theoretically enabled engineering the defects for different precursor Mo/S molarity ratios of 4:2, 4:4 and 4:8 as elucidated in Fig. 17c [149]. Consequently, the Mo/S ratio of $4: 2$ provided the highest amount of defects in the crystal shown as $\operatorname{MoS}_{2} \mathrm{D}_{\mathrm{H}}$ in Fig. 17c [149]. Xie et al. developed a scalable pathway to engineering defects in $2 \mathrm{D} \mathrm{MoS}_{2}$ using a high concentration of precursors and different amounts of thiourea. The thiourea was used both to reduce Mo(vi) to Mo(iv) as well as stabilising the morphology [150]. The number of active sites of defect-rich 2D $\mathrm{MoS}_{2}$ was then engineered by adjusting the concentrations of precursors and thiourea and reached 13 times more than that of bulk $1.785 \times 10^{-3} \mathrm{~mol} \mathrm{~g}^{-1}$ (Fig. 17d) [150]. Yin et al. developed liquid-ammonia-assisted lithiation chemical synthesis to produce metallic $1 \mathrm{~T}$ phase $\mathrm{MoS}_{2}$ with active edge sites and sulphur vacancies. The defects from the chemical synthesis include holes as shown in Fig. 17b [148]. Generally, in transition metal sulphides, sulphur deficiencies create n-type doping and transition metal deficiency causes p-type doping which can be achieved by adjusting precursor ratios and stoichiometries. As a result of this adjustment, different intrinsic 0D defects can form during CVD synthesis which will be explored in Sect. 4.2. Besides intrinsic defects during synthesis, the defects can be generated post-synthesis intentionally using plasma, ion/electron beam, laser and sputtering [151-158] which can potentially be used for creating large-area 2D heterojunctions and local sites with spin-orbit effects for
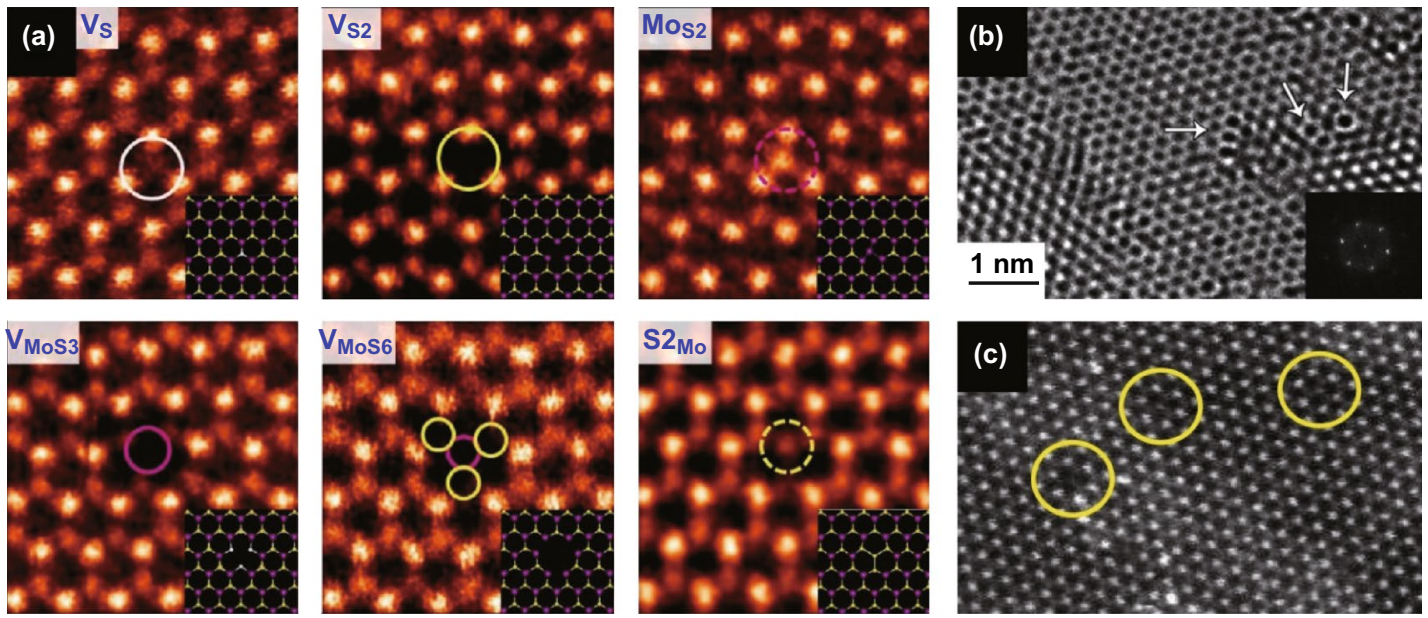

Fig. 16 Intrinsic 0D defects of 2D TMDCs during the CVD growth. a Annular dark-field (ADF) images of CVD grown of MoS ${ }_{2}$ monolayer. Point defects and fully relaxed structural model (inset) of mono-sulphur vacancy $\left(\mathrm{V}_{\mathrm{S}}\right)$, disulphur vacancy $\left(\mathrm{V}_{\mathrm{S} 2}\right)$, antisite defects where a Mo atom substituting an $\mathrm{S}_{2}$ column $\left(\mathrm{Mo}_{\mathrm{S} 2}\right)$, vacancy complex of Mo and nearby three sulphur $\left(\mathrm{V}_{\mathrm{MoS} 3}\right)$, vacancy complex of Mo nearby three disulphur pairs $\left(\mathrm{V}_{\mathrm{MoS} 6}\right)$, and a $\mathrm{S}_{2}$ column substituting a Mo atom $\left(\mathrm{S}_{\mathrm{Mo}_{\mathrm{Mo}}}\right)$. Purple, yellow and white circles indicate Mo, top layer $\mathrm{S}$ and bottom layer $\mathrm{S}$, respectively. Adapted with permission from Ref. [141]. Copyright 2013, ACS Publications. b HRTEM images of point defects in 2D WS 2 structure generated during growth of the oxide and consequent conversion to sulphide. Inset shows the corresponding fast Fourier transform (FFT) of the TEM micrograph. Adapted with permission from Ref. [143]. Copyright 2013, ACS Publications. c HRTEM micrograph of a 2D WS 2 grown by thermal reduction/sulphurisation method with yellow circles highlighting the intrinsic point defects. Adapted with permission from Ref. [142]. Copyright 2015, ACS Publications 

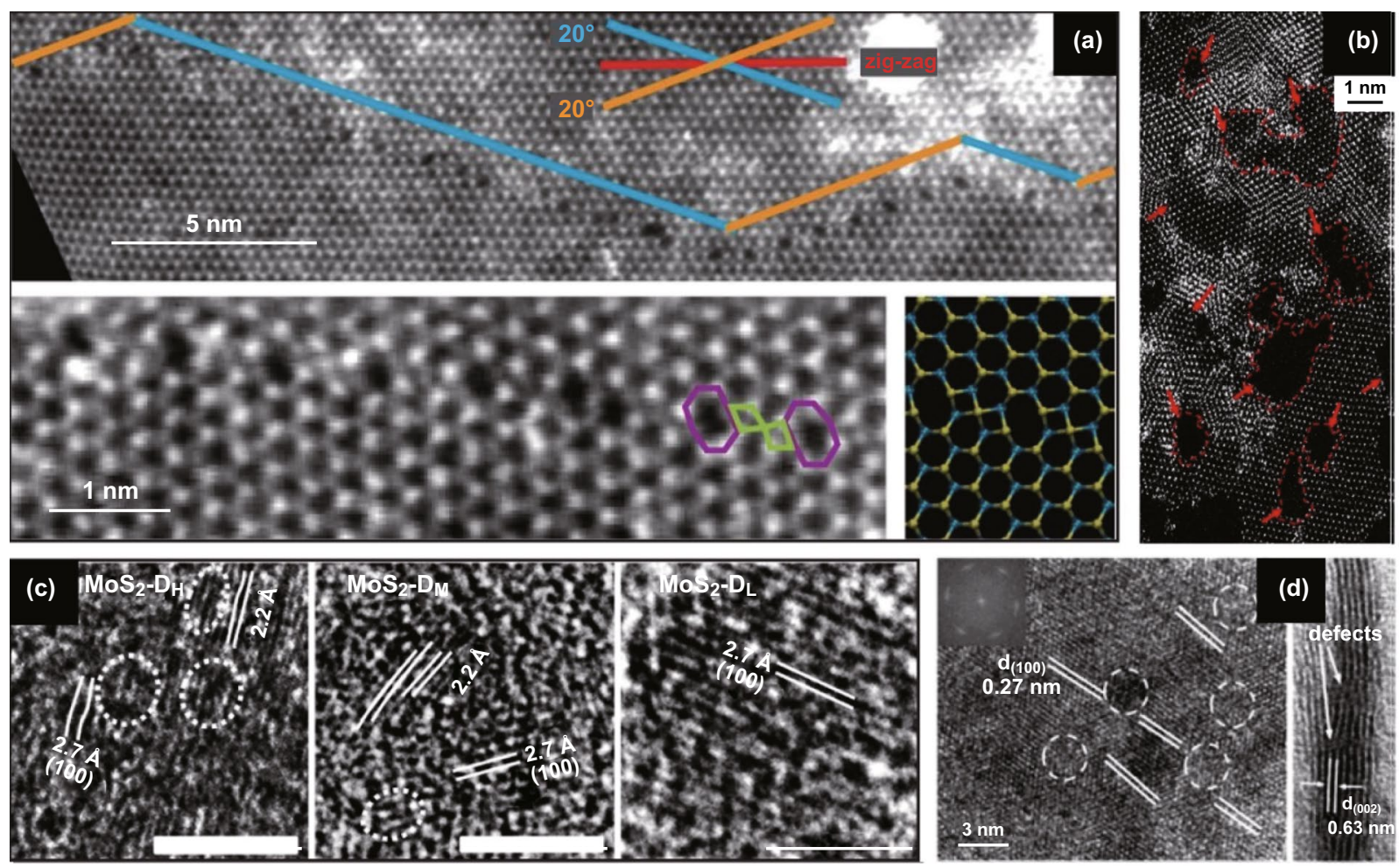

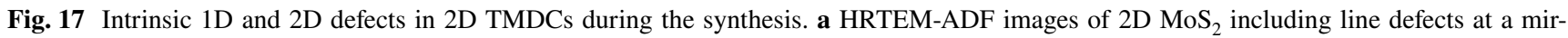
ror twin grain boundary (top). Below is the zoomed-in image shows a periodic line of 8-4-4 ring defects along the grain boundary, including an atomistic model on the right. Adapted with permission from Ref. [147]. Copyright 2019, ACS Publications. b Mesoporous (holey) 1 T-MoS 2 nanosheet with two-dimensional defects many edge sites synthesised with severe desulphurisation reaction condition between lithium and $\mathrm{MoS}_{2}$. Adapted with permission from Ref. [148]. Copyright 2015, ACS Publications. c HRTEM images of engineered sulphur deficient MoS show $_{2}$ dislocations and distortions of lattice planes decreases (left to right) from reaction precursor Mo/S ratios of 4:2 $\left(D_{H}\right)$, 4:4 $\left(D_{M}\right)$ and 4:8 $\left(D_{L}\right)$. Adapted with permission from Ref. [149]. Copyright 2019, Nature Publishing Group. d HRTEM micrograph of defect-rich structure of MoS 2 and active edge sites generated by varying precursors during the synthesis process. Adapted with permission from Ref. [150]. Copyright 2013, Wiley

applications in high-performance optoelectronics and quantum computing.

\subsection{The Influences of Defects on the Electronic and Optical Properties of 2D Materials}

Several properties of 2D materials are affected by the defects including optical, electronic, magnetic, chemical, vibrational and thermal. The grain boundaries and defects hinder electronic performances, including transport [159], which largearea 2D materials consequently affected critically from their presence. However, reports indicate the presence of defects and less-ordered crystals can potentially promote highly efficient and fundamentally novel electronic and optoelectronic devices [160].
Yu et al. demonstrated n-type doping $\mathrm{WS}_{2}$ as a result of structural defects generated during the CVD process [144]. In addition to electronics n-type doping, the induced charge defects enabled by the structural imperfection changed the optical behaviour produced PL quenching and blue shift in some regions of the synthesised $2 \mathrm{D} \mathrm{WS}_{2}$ flakes (Fig. 18a-d) [144].

Van der Zande et al. produced large grain sizes of $\mathrm{MoS}_{2}$, enabling the study of boundary defects. Two distinct PL was observed corresponding to different doping types of crystal at boundaries. The mirrored boundary line defects with 8-4-4 membered ring structures are Mo rich giving rise to n-type doping, and on the other hand, the tilt boundary line defects with 5-7 membered ring structures are $S$ rich giving rise to p-type doping of the grain boundaries. This, in turn, will cause PL quenching/enhancement with 

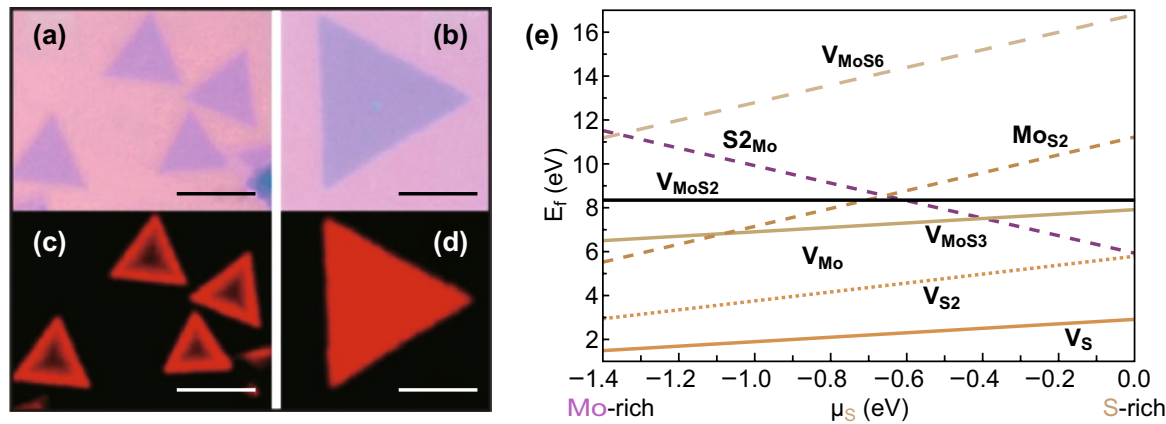

(f)
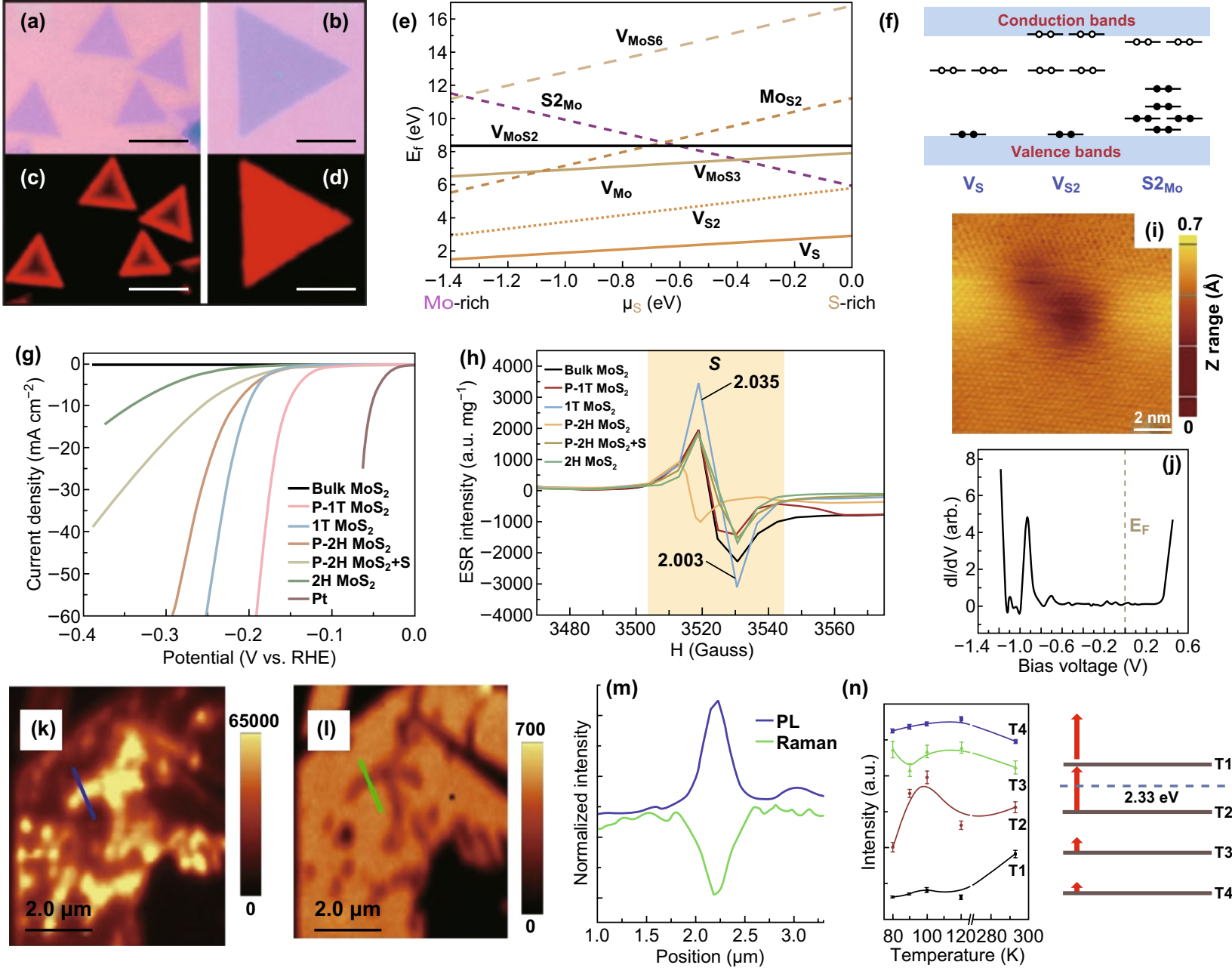

(n)
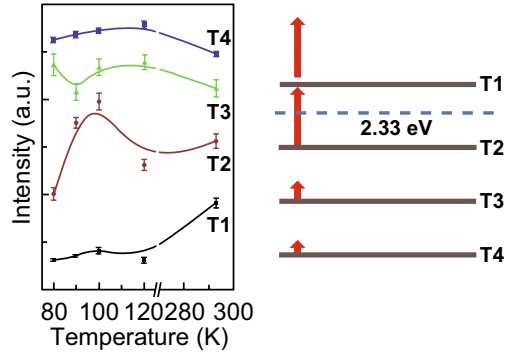

Fig. 18 Defect-driven properties of 2D materials. a, b Optical images and $\mathbf{c}, \mathbf{d}$ corresponding fluorescence images of monolayer CVD grown WS. Adapted with permission from Ref. [144]. Copyright 2013, ACS Publications. e Chemical potentials to create point defects elucidated in Fig. 16a, indicate which defects are more likely to occur and $\mathbf{f}$ the corresponding schematic of the electronics structural in-gap defect states. Adapted with permission from Ref. [141]. Copyright 2013, ACS Publications. g, h Electrochemical characterisation of various defective $\mathrm{MoS}_{2}$ compared with Pt and the corresponding electron spin resonance with a higher intensity for samples with less sulphur vacancy defect. Adapted with permission from Ref. [148]. Copyright 2016, ACS Publications. i, j STM image of a twin boundary defect and its STS showing an in-gap state at $-0.94 \mathrm{~V}$. Adapted with permission from Ref. [161]. Copyright ACS Publications. $\mathbf{k}$ PL enhancement and $\mathbf{I}$ Raman quenching at a 1D crack defect in $\mathrm{MoS}_{2}$ and $\mathbf{m}$ the corresponding spectra. Adapted with permission from Ref. [162]. Copyright 2014, ACS Publications. $\mathbf{n}$ Raman intensity of $A_{(}^{\prime}(\Gamma)$ phonon mode of CVD grown $\mathrm{WS}_{2}$ monolayers at four different temperatures (T1-T4). Samples are synthesised with increasing defect densities showing distinct Raman intensities and excitonic energy differences. Adapted with permission from Ref. [138]. Copyright 2019, ACS Publications

increase/decrease in electron density, respectively [146]. Interestingly, the mirror boundary defects reduced PL quantum yield, and in contrast, tilt boundary defects enhanced PL quantum yield [146]. This result indicates a significant effect of defects on optical electronics properties from being n-type to p-type semiconductor. In addition to the diverse doping type effects, various point defects that are shown in Fig. 16a-d are demonstrated to be more favourable to form under different conditions (Fig. 18e). These 0D defects can create in-gap states as shown in Fig. 18f [141]. Electronic transport characteristics are shown to be affected by localised trap states caused by defects and grain boundaries [145, 163]. As a result, many of the electronics and optoelectronics properties can substantially be influenced by defects.

Similar to CVD grown defects, increasing defect using ion bombardments of TMDCs lead to PL intensity quenching 
[138, 164-166]. Raman intensity dependency at sulphur vacancies in $\mathrm{MoS}_{2}$ is shown to create a pronounced in-gap state measured by scanning tunnelling microscopy for ME $2 \mathrm{H}-\mathrm{MoS}_{2}$ (Fig. 8i, j) [161]. The density of states calculations for $\mathrm{MoS}_{2}$ and $\mathrm{WS}_{2}$ confirms crystals showing this property due to the point defects [167]. The bandgap of alloy film of $\mathrm{MoS}_{2(1-\mathrm{x})} \mathrm{Se}_{2 \mathrm{x}}$ was successfully engineered from 1.87 and $1.55 \mathrm{eV}$ by tuning $\mathrm{x}$ from 0 to 1 [168]. The ON-current, motility and resistance in $\mathrm{MoS}_{2}$ are defect controlled with oxygen-argon plasma irradiation up to four orders of magnitude [153]. The surface-induced defects may serve as an ambipolar charge trapping layer [155]. Defects generated by proton irradiation reduced the current and conductance of a multilayer $\mathrm{MoS}_{2}$ FET device [156].

Point defects in $\mathrm{MoS}_{2} / \mathrm{WS}_{2}$ created with replacements of $\mathrm{S}$ with $\mathrm{O}$ are demonstrated to change wetting behaviour of the TMD film to become more hydrophobic [142]. Xie et al. [150] engineered the chemical reaction for the synthesis of $\mathrm{MoS}_{2}$ to generate defects using different concentrations of precursors and thiourea and effectively increased the catalytically active edge sites. Electrochemical performance of the defective 2D TMDCs with active edge site is shown to significantly improve the catalytic performances during the hydrogen evolution reaction [148, 150, 169].

Magnetic properties of TMDCs are shown to be affected by defects from the reduction in the intensity of electron spin resonance spectra of $\mathrm{MoS}_{2}$ as a result of S-vacancies [148]. Jin et al. [148] demonstrated porous $1 \mathrm{~T} / 2 \mathrm{H}$ phases of $\mathrm{MoS}_{2}$ with significantly less intensity of electron spin resonance than that of conventional $1 \mathrm{~T}$ phase $\mathrm{MoS}_{2}$ (Fig. 18g, h).

Raman study of $\mathrm{Ar}^{+}$plasma irradiated of $\mathrm{MoS}_{2}$ shows a weakening of the interlayer interactions as well as dielectric properties resulting in blue shift to $E_{2 g}^{1}$ peak which is speculated to be as a result of structural defects [170]. On the other hand, $\mathrm{A}_{1 \mathrm{~g}}$ peak is blue-shifted due to p-typed doping as a result of stronger oxygen bonds due to the annealing induced cracks and imperfections (Fig. 18k-m) [162]. Raman scattering intensity is shown to be proportional to the density of defects providing a route to quantify the defects in monolayer $\mathrm{MoS}_{2}$ [171]. Thermal conductivity of the $\mathrm{MoS}_{2}$ is shown to increase with defect mediated gold nanoparticle incorporation. The carrier transport thermal barrier was reduced 5.7 times after functionalisation through the defect sites [172]. Defect densities in a monolayer of $\mathrm{WS}_{2}$ are demonstrated to directly change excitonic binding energy by up to $110 \mathrm{meV}$ and affect phonon-exciton interactions (Fig. 18n) [138]. Defects have profound effects on various properties of 2D materials which is necessary to realise for the design of electronics, optoelectronics and quantum-confined enabled devices.

\subsection{Strategies for Enhancing Crystal Domain Size}

Currently, large-area uniform 2D materials with minimum defects and grain boundaries are readily available through extensive research and synthesis optimisations over more than a decade. Several synthetic routes, including CVD, MOCVD, ALD, PLD, MBE, ME and LM, have been explored. However, most advancements and knowledge have been developed in CVD synthesis due to a prime focus being dedicated to this method. Some of the recent techniques that are employed to perfect the synthesis strategies including the effect of substrate facet, selection and preconditioning, carrier gas mixture and impurities, the influence of precursor quantity and morphology and thermodynamics engineering for effective control of the growth kinetics are discussed here.

\subsubsection{Substrate Effects}

CVD method is substrate sensitive [27]. Li et al. exploited the balance between the symmetry of grown $\mathrm{hBN}$ and substrate $\mathrm{Cu}(110)$ to obtain $100 \mathrm{~cm}^{2}$ single-crystal monolayer of $\mathrm{hBN}$. The authors resolved a major problem of the CVD process regarding the formation of twin boundary defects due to the coalescence of the triangular-shaped grains with different crystallographic orientations [17, 92]. Inspired by crystal facet engineering, nucleation of $\mathrm{hBN}$ is shown to initiate at $\mathrm{Cu}(211)$ edge, which is coupled with the hBN zigzag crystal structure. It is also theoretically confirmed that the edge coupling is an energetically more favourable arrangement [17]. Alloying $\mathrm{Cu}$ with $\mathrm{Ni}$ as substrate, on the other hand, has resolved crystal orientation requirements for wafer-scale production of graphene, which is relied on evolutionary growth of favourable crystal domain [127]. Using liquid metals as substrates is an emerging method for producing large-area single crystal which is demonstrated 
for hBN growth on liquid Au (Fig. 5) [35]. This process offers full coverage of up to several centimetres with smaller domains joining to create a large-area crystal optimised with respect to time [35]. Liquid metal melts such as $\mathrm{Cu}$ as a substrate produce self-aligned $\mathrm{hBN}$ domains and in case of graphene, minimised grain boundary formation, respectively [76, 129]. Substrate effects, such as pre-treatment with rGO, perylene tetracarboxylic acid tetra potassium salt and perylene tetracarboxylic dianhydride to use molecular agglomerates as controlled seed sites, provide controlled growth of $\mathrm{MoS}_{2}$ for up to several centimetres on the amorphous $\mathrm{SiO}_{2}$ substrate [6].

\subsubsection{Precursor Effects}

Precursor quantity has profound effects on CVD synthesis during nucleation and growth of the crystals. Lee et al. [25] fundamentally explored this effect by spin coating $\mathrm{MoO}_{3}$ precursors on substrates and placing them above the destination $\mathrm{MoS}_{2}$ substrate. It was realised that excessive precursor amounts resulted in the increase in nucleation rates due to supersaturation of precursors. Consequently, the grain sizes were reduced (the blue shaded right region in Fig. 19a). The authors separated this regime from a thermodynamically stable nucleation regime (the pink shaded left region in Fig. 19a) when the precursor amounts are optimised [25].
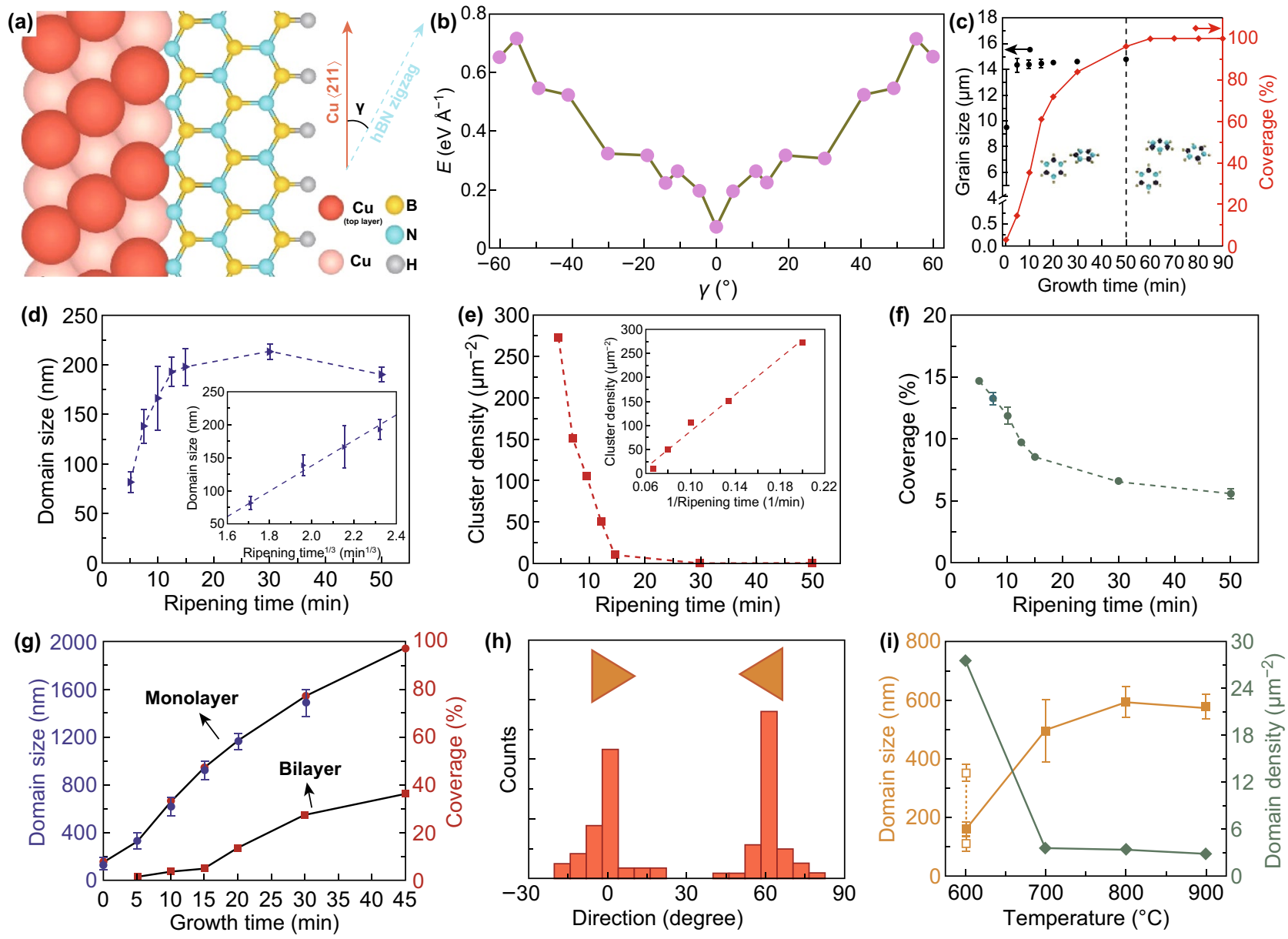

Fig. 19 Strategies for enhancing crystal domain sizes. a Effect of the amount of precursor on nucleation and growth during CVD synthesis of $\mathrm{MoS}_{2}$ to provide optimum crystal domain size. Adapted with permission from Ref. [25]. Copyright 2017, Wiley. b, c Oxygen-assisted CVD growth of $\mathrm{MoS}_{2}$ leads to an increase in domain size in $30 \mathrm{~min}$; however, domains start to etch away if growth time is increased further. Adapted with permission from Ref. [56]. Copyright 2015, ACS Publications. d-f Diffusion-controlled growth of WSe ${ }_{2}$ by optimising ripening step showing domain size, cluster density and substrate coverage as a function of ripening time. $\mathbf{g}, \mathbf{h}$ Effect of growth step time on area coverage highlighting the domain direction statistics. i Effect of substrate temperature on domain size and density. Adapted with permission from Ref. [173]. Copyright 2018, ACS Publications 
This phenomenon was previously observed by Najmaei et al. [55] to realise the effect of $\mathrm{MoO}_{3}$ nanoribbon precursor dispersion to adjust nucleation rate and growth. The authors fully characterised the crystal quality, considering the formation of the most common defects in $2 \mathrm{D}$ crystals entailing OD and 1D defects. Creation of these defects was analysed during the CVD growth of $\mathrm{MoS}_{2}$ [55]. The nucleation and growth were controlled by two CVD parameters of precursor concentration and pressure to produce large-area and grainboundary-free $\mathrm{MoS}_{2}$ monolayers. Grain boundary and 5-7 ring defects were used for identifying the mechanism that lies in nucleation, and growth of one-dimensional line defect grain boundary [55].

\subsubsection{Carrier Gas Mixture Effects}

Favourable effects of different gas mixtures in CVD processes are explored. As discussed, $\mathrm{H}_{2}$ gas effectively activates oxide precursor conversion during selenisation process [27]. When metal is used as a precursor, the removal of oxygen during CVD synthesis is shown to enhance the stability of transition metal selenides [174]. However, Chen et al. [56] demonstrated oxygen-assisted synthesis when transition metal oxides are used as the precursor. Therefore, it is noteworthy to devise a suitable carrier gas mixture according to the type of the precursor used. The presence of oxygen is shown to effectively prevent the oxide precursor from poisoning, which is premature sulphurisation of oxide during the evaporation stage and eliminates the formation of defects during the synthesis [56]. The premature sulphurisation occurs when sulphur reacts with $\mathrm{MoO}_{3}$ and prevents continuous evaporation of $\mathrm{MoO}_{3}$. In addition, oxygen etches away the unstable nuclei and prevents the formation of nanotubes and nanoparticles. Figure 19b, c elucidates optimisation of domain size and growth rates in the presence of a low oxygen flow rate [56].

\subsubsection{Thermodynamics Effects}

Recently, Zhang et al. [173] fundamentally investigated the surface diffusion effect on lateral growth of $\mathrm{WSe}_{2}$. The authors systematically separated the growth process into three distinct steps, including nucleation, ripening and lateral growth. In the first step, precursors are nucleated at a high flow rate and short duration of $30 \mathrm{~s}$, followed by an annealing ripening step with $\mathrm{H}_{2} \mathrm{Se}$ gas [173]. As shown in Fig. 19d-f during the ripening step, domain sizes increased by diffusion of the $\mathrm{W}$ adatoms and migration of $\mathrm{WSe}_{\mathrm{x}}$ clusters. Consequently, cluster density and substrate coverage decreased. Finally, precursors were reintroduced at an optimised flow rate for lateral growth and full coverage of the substrate (Fig. 19g, h). This multi-step process entailing nucleation, ripening and lateral growth steps enabled a fundamental study of nucleation and growth in detail. As such, the authors show the effect of substrate temperature on the domain size and density during the growth step as elucidated in Fig. 19i [173].

The crystal quality has been rigorously optimised in CVD processes; however, other methods are lacking protocols to obtain large-area single crystals. The investigation should be a focus of future explorations for other synthesis methods.

\section{Electronic and Optoelectronic Performances of Large-Area Synthesised 2D Semiconductors}

Each synthesis method conventionally presents with challenges; for example, CVD and MBE both suffer mostly from grain boundary defects and LM methods from liquid metal inclusions during the transfer. Nevertheless, several high performing devices have been reported using these methods including a design of a complete logical circuit enabled by the large-area synthesis of 2D materials [126]. Many promising optoelectronics components have been synthesised such as FET and photodetectors which are summarised below. CVD grown $\mathrm{Bi}_{2} \mathrm{O}_{2} \mathrm{Se}$ features ultra-high mobility with on/off ratios $\left(>10^{6}\right)$ at room temperature for single crystal with sizes exceeding $200 \mu \mathrm{m}$ [45]. Fieldeffect transistors (FET) based on CVD synthesised $\mathrm{MoTe}_{2}$ with high-quality crystals have been made featuring on/off ratios of $\sim 1000$ and carrier mobility of $1 \mathrm{~cm}^{2} \mathrm{~V}^{-1} \mathrm{~s}^{-1}$ [3]. Large-area $\mathrm{WSe}_{2}$ single crystal with areas of $\sim 100,000 \mu \mathrm{m}^{2}$ demonstrates high hole mobility of $102 \mathrm{~cm}^{2} \mathrm{~V}^{-1} \mathrm{~s}^{-1}$ [30]. Lan et al. [26] reported large-area growth of $\mathrm{WS}_{2}$ with low mobility of $\sim 0.02 \mathrm{~cm}^{2} \mathrm{~V}^{-1} \mathrm{~s}^{-1}$ associated with the formation of OD defects to low mobility due to increased scattering of charges. The summary of electrical performances of largearea synthesised 2D semiconductors is shown in Table 1 . As a benchmark for high-quality exfoliated 2D materials, mechanically exfoliated $\mathrm{MoS}_{2}$ has room temperature 
Table 1 Electrical performances of large-area 2D materials

\begin{tabular}{|c|c|c|c|c|c|}
\hline Material & Method & On/Off & Mobility $\left(\mathrm{cm}^{2} \mathrm{~V}^{-1} \mathrm{~s}^{-1}\right)$ & Bandgap (eV) & Refs. \\
\hline $\mathrm{Bi}_{2} \mathrm{O}_{2} \mathrm{Se}$ & LPCVD & $>10^{6}$ & 450 at RT 29,000 at $1.9 \mathrm{~K}$ & 0.8 & {$[45]$} \\
\hline $\mathrm{MoTe}_{2}$ & APCVD & 1000 & 1 at RT & - & [3] \\
\hline $\mathrm{MoS}_{2}$ & LPCVD & $6 \times 10^{6}$ & 4.3 at $\mathrm{RT}$ & - & {$[55]$} \\
\hline $\mathrm{MoS}_{2}$ & APCVD & $8 \times 10^{8}$ & 24 at RT $8420 \mathrm{~K}$ & - & {$[57]$} \\
\hline $\mathrm{MoS}_{2}$ & LPCVD & $6 \times 10^{6}$ & $\begin{array}{l}30 \text { at RT } \\
114 \text { at } 90 \mathrm{~K}\end{array}$ & 1.9 & {$[1]$} \\
\hline $\mathrm{ReS}_{2}$ & APCVD & 1000 & - & 1.59 & [4] \\
\hline $\mathrm{WSe}_{2}$ & APCVD & $10^{7}$ & hole (102) electron (26) at RT & 1.65 & {$[30]$} \\
\hline $\mathrm{WS}_{2}$ & APCVD & $10^{7}$ & electron (14) at RT & 1.99 & {$[30]$} \\
\hline $\mathrm{WS}_{2}$ & LPCVD & $10^{6}$ & 0.91 at RT & 1.9 & {$[83]$} \\
\hline $\mathrm{WS}_{2}$ & LPCVD & $5.5 \times 10^{3}$ & 0.02 at RT & 2 & {$[26]$} \\
\hline $\mathrm{WSe}_{2}$ & PLD & 103 & 0.00528 at RT & - & {$[33]$} \\
\hline $\mathrm{MoS}_{2}$ & ME & - & 26 at RT & - & {$[24]$} \\
\hline $\mathrm{ZnO}$ & AILE & - & hole $(0.10)$ at RT & 2.53 & [49] \\
\hline $\mathrm{GaN}$ & LM-PCVD & - & 21.5 at RT & 3.5 & {$[51]$} \\
\hline $\mathrm{SnO} / \mathrm{In}_{2} \mathrm{O}_{3}$ & LM & - & 37 at RT & $4.08 / 3.65$ & {$[16]$} \\
\hline $\mathrm{Ga}_{2} \mathrm{~S}_{3}$ & LM & 100 & 3.5 at RT & 2.1 & {$[52]$} \\
\hline $\mathrm{GaS}$ & LM & 150 & 0.2 at $\mathrm{RT}$ & 3.1 & [13] \\
\hline $\mathrm{SnO}$ & LM & 300 & 0.7 at RT & 4.2 & [175] \\
\hline $\mathrm{In}_{2} \mathrm{~S}_{3}$ & LM & $10^{4}$ & 58 at RT & 2 & [71] \\
\hline
\end{tabular}

mobilities of greater than $200 \mathrm{~cm}^{2} \mathrm{~V}^{-1} \mathrm{~s}^{-1}$ [176], however, in large-scale fabrication using most common CVD methods charge mobilities falls short in performances [1, 3, 55, 57].

Larger area 2D materials provide a higher effective surface for optoelectronic devices, therefore, enhancing performances. The large area can accommodate more components for integrated optoelectronics circuits as well as allowing the design of larger gaps between electrodes. Suitable bias voltages are needed to be selected to operate and characterise the optoelectronics devices when changing the distance between electrodes to incorporate the impedance variations [16].

High responsivity photodetection with fast response times is reported for large-area devices produced by the LM method, as presented in Table 2. Photodetectors with ultrasensitive and high detectivity of $10^{13}$ Jones and wide spectral

Table 2 Optoelectronic performances large-area 2D materials

\begin{tabular}{|c|c|c|c|c|c|c|c|c|}
\hline Materials & Method & Thickness & Lateral size & Responsivity $\left(\mathrm{A} \mathrm{W}^{-1}\right)$ & Detectivity (Jones) & $\begin{array}{l}\text { Response } \\
\text { time }(\mathrm{ms})\end{array}$ & Spectral range $(\mathrm{nm})$ & Refs. \\
\hline $\mathrm{BiOI}$ & APCVD & Few layers & $>100 \mu \mathrm{m}$ & 0.026 & $8.2 \times 10^{11}$ & 120 & 473 & {$[46]$} \\
\hline $\mathrm{MoS}_{2}$ /graphene & APCVD & $1 \mathrm{~L}$ & $\mathrm{~cm}$ & 2.4 & - & - & 532 & [54] \\
\hline $\mathrm{PdSe}_{2}$ & APCVD & 1 to few layers & $\mathrm{cm}$ & 0.3 & $10^{13}$ at $780 \mathrm{~nm}$ & - & Up to $\sim 1100$ & [28] \\
\hline $\mathrm{WS}_{2}$ & LPCVD & $1 \mathrm{~L}$ & $\mathrm{~cm}$ & 18.8 & - & 4.5 & 532 & [83] \\
\hline $\mathrm{WS}_{2}$ & LPCVD & $1 \mathrm{~L}$ & $\mathrm{~cm}$ & 0.005 & $4.9 \times 10^{9}$ & 560 & 532 & [26] \\
\hline $\operatorname{ReS}_{2}$ & APCVD & $1 \mathrm{~L}$ & $\mathrm{~cm}$ & 278 & - & - & 405 & [4] \\
\hline $\mathrm{Bi}_{2} \mathrm{O}_{3}$ & LM & $1 \mathrm{~L}$ & $\mathrm{~cm}$ & 400 & $\begin{array}{l}1.1 \times 10^{13} \\
\text { at } 365 \mathrm{~nm}\end{array}$ & 4.3 & 365 & {$[15]$} \\
\hline $\mathrm{SnO} / \mathrm{In}_{2} \mathrm{O}_{3}$ & LM & $1 / 4.5 \mathrm{~nm}$ & $\mathrm{~mm}$ & $1047,600,173$ & $5 \times 10^{9}$ at $280 \mathrm{~nm}$ & 1 & $280,365,455$ & {$[16]$} \\
\hline $\mathrm{Ga}_{2} \mathrm{~S}_{3}$ & LM & $2 \mathrm{~nm}$ & $\mathrm{~cm}$ & 240 & $10^{10}$ at $455 \mathrm{~nm}$ & 100 & $365,455,565$ & [52] \\
\hline $\mathrm{In}_{2} \mathrm{Se}_{3}$ & $\mathrm{CVP}$ & $3.6 \mathrm{~nm}$ & $>200 \mu \mathrm{m}$ & 5.6 & $7 \times 10^{9}$ at $660 \mathrm{~nm}$ & 140 & $365-850$ & [177] \\
\hline
\end{tabular}


ranges are reported for $\mathrm{PdSe}_{2}$ synthesised in centimetre scale with uniform thicknesses [28]. In addition, large-area devices enable more effective scientific investigations for intriguing properties of 2D materials. As such, Chen et al. [54] demonstrated the quench of photoluminescence (PL) in the large-area grown $\mathrm{MoS}_{2}$ when forming a heterojunction with graphene due to charge transfer at the interface. Huang et al. have shown large-area grown $\mathrm{WSe}_{2}$ with an indirect gap absent in monolayer. Instead, only PL emissions at A and $\mathrm{B}$ excitonic absorptions are seen, corresponding to the direct bandgap of a monolayer [27].

A significant prospective optoelectronics application of large-area 2D materials is transparent and conductive wide bandgap semiconductors enabling large display panels as well as flexible and stretchable electronics. As the thickness of transparent and conductive wide bandgap semiconductors such as ITO is reduced, the light absorption spectra are shown to decrease indicating a potential to be incorporated as a top contact in solar panels and smartphones to enhance performances, providing better brightness and lowering the power consumption [47]. Large-area printed 2D materials enable miniaturised electronic components and to fit more components into devices as shown in Fig. 20a, 8100 FET devices are fabricated within a monolayer of $\mathrm{MoS}_{2}$ [1]. Multi-component logical devices are shown to be fabricated from heterostructures of large-area $\mathrm{MoS}_{2}$ monolayer (Fig. 20b) [126]. Large-area photodetectors are reported with excellent detectivities (Fig. 20c, d and g) suggesting promising pathways towards high-efficiency devices [16, 26, 83, 177]. Large-area printing of atomically thin materials enables fabrication of multiple electronics devices resulting in the precise and more in-depth statistical analysis of devices [13, 33, 83]. LM synthesis of large-area GaS is presented in Fig. 20e. These layers are achieved by screen printing of molten gallium to transfer the surface oxides onto a $\mathrm{SiO}_{2}$ wafer, followed by chemical conversion and sulphurisation [13]. PLD methods that can potentially be used to produce a variety of large area are shown to produce $\mathrm{WSe}_{2}$ with high uniformity (Fig. 20f) [33].

Emerging 2D magnetic materials for potential application in spintronics, valleytronics and twistronics with large lateral dimensions have rarely been realised. Chu et al. [58] synthesised vdW epitaxial growth of single-crystal $\mathrm{Cr}_{2} \mathrm{~S}_{3}$ in a single unit cell exceeding $200 \mu \mathrm{m}$. This material feature air-stable p-type semiconductor ferromagnet with intriguing properties. Yu et al. synthesised $2 \mathrm{D} \mathrm{VSe}_{2}$ using exfoliation electrochemically to produce atomically thin layers with strong ferromagnetic properties at high curie temperatures for potential memory device applications [59]. Development of such large-area 2D magnetic materials is of interest for applications in quantum computing which is the currently lacking literature.

\section{Conclusions}

The quest for the synthesis of large-area atomically thin 2D materials with uniform thicknesses and minimum structural defects has effectively led to many successful reports and emerging strategies. This topic is the subject of extensive and ongoing research presenting several performance and scalability challenges to be adopted by industry. One major drawback in the development of large-area high-quality 2D materials is the lack of spectroscopic solutions for analysing the quality of the obtained large-area 2D materials in atomic resolution in a single measurement. Current methods to capture HRTEM at atomic resolution for centimetre-scale 2D materials are performed through stitching images and locally verifying the grain boundary sizes. In addition, electron irradiation during TEM has found to introduce defects in 2D materials even at relatively low acceleration voltages of 80 and $60 \mathrm{kV}[151,152]$. Besides the adverse effect of TEM in introducing defects, Raman laser is also shown to generate defect in $\mathrm{WSe}_{2}, \mathrm{TaS}_{2}$ and $\mathrm{TaSe}_{2}$ nanosheets by damaging the crystal and oxidisation $[179,180]$. The uniformity assessment of 2D materials is measured locally using limited area AFM image and generalised to centimetre-scale grown 2D materials using an optical microscope, which is none ideal method of characterising large-area 2D materials.

Among synthesis methods, top-down approaches, such as $\mathrm{ME}$, are low cost and produce high-quality exfoliated 2D sheets exceeding half a millimetre in lateral dimensions, however, lacking scalability and yield [20]. Successful bottom-up approaches such as CVD have shown many promises to produce large-area single-crystal 2D materials including hBN $[17,35]$. The breakthroughs in CVD synthesis have been achieved by substrate facet engineering or using liquid metals as substrate. The former requires lattice matching between substrate edge, which requires extended investigation for other $2 \mathrm{D}$ materials with different crystal structures than that of hBN. The latter needs an inert metal melt as a substrate and requires the synthesis at temperatures 

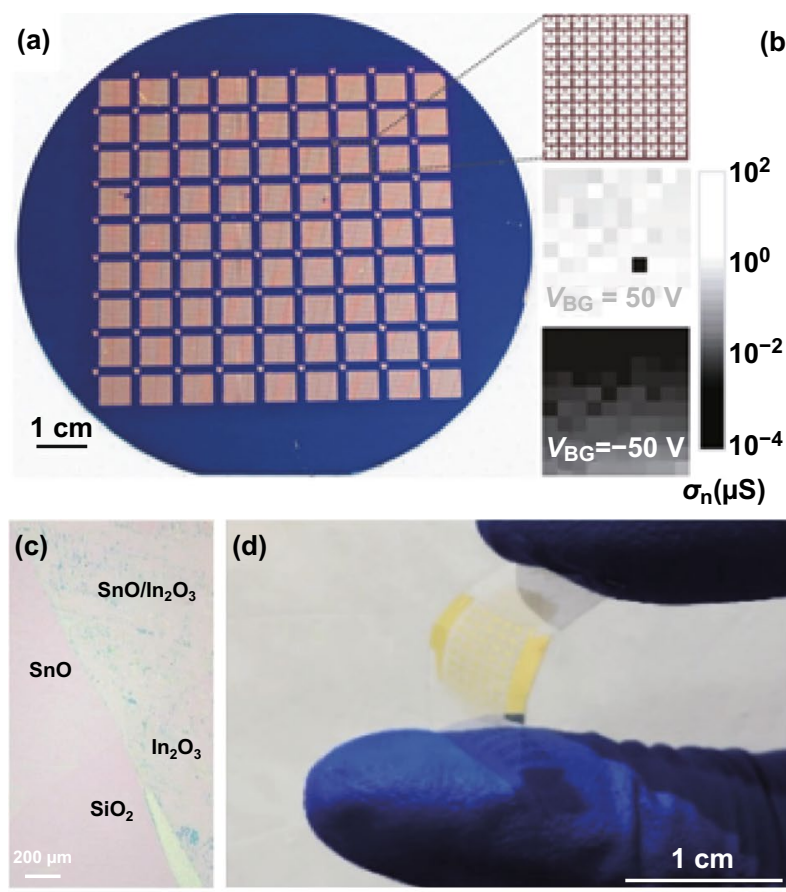

(d)
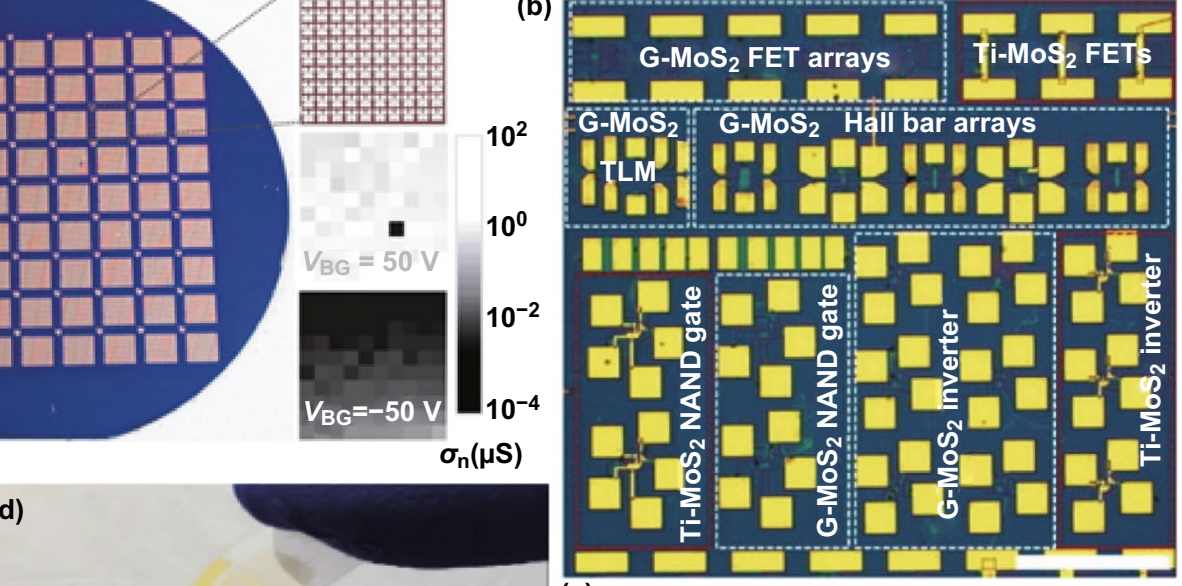

(e)
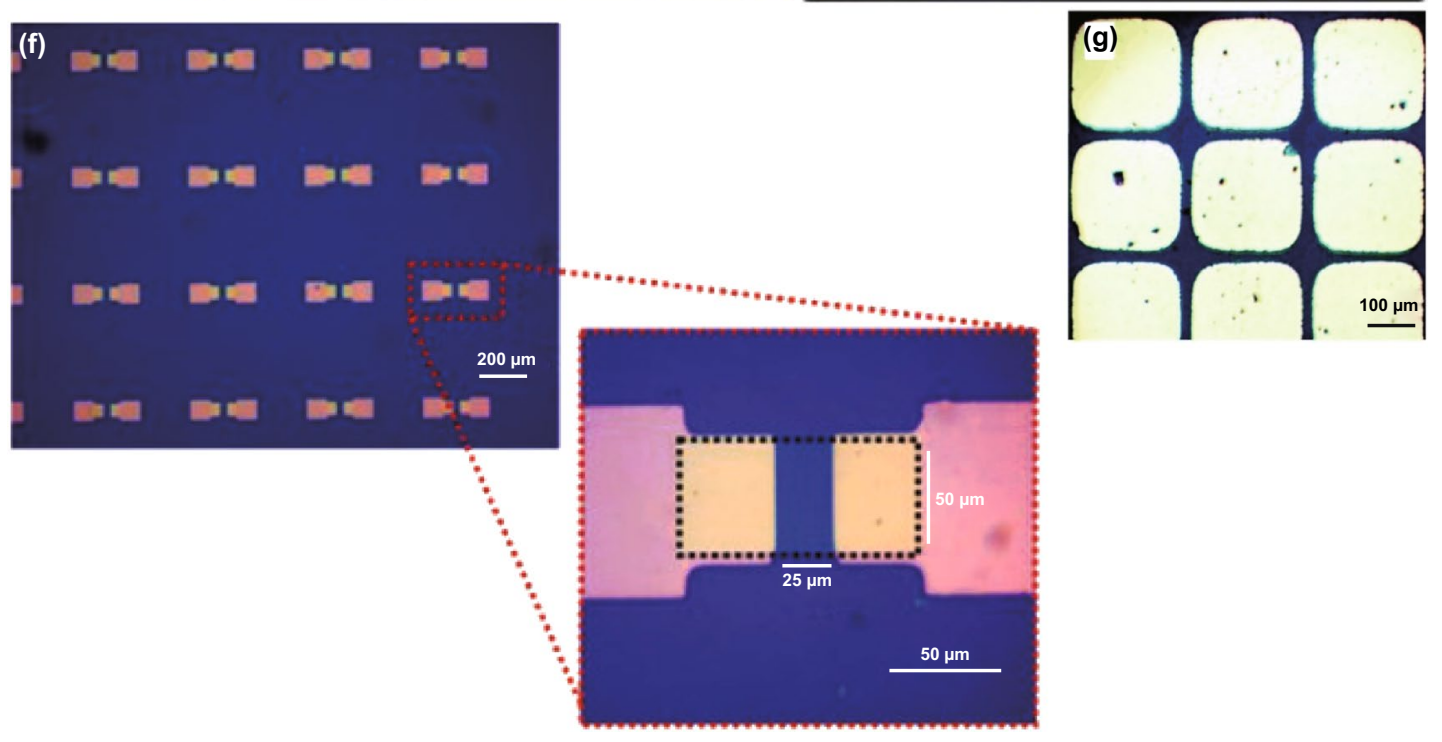

Fig. 20 2D large area enabled optoelectronics applications. a 8100 FET devices from synthesised large-area monolayer of MoS 2 The top inset shows area with 100 FET devices. Bottom insets indicate one non-functional device found in the 100 shown devices when $50 \mathrm{~V}$ is applied to the gate. Adapted with permission from Ref. [178]. Copyright 2019, MDPI publishing. b Optical image of a large-area integrated chip from MoS monolayers including graphene and Ti/Au electrodes. Scale bar is $500 \mu \mathrm{m}$. Adapted with permission from Ref. [126]. Copyright 2014, ACS Publications. c vdW oxide heterostructures synthesised from LM methods in large scale. Adapted with permission from Ref. [16]. Copyright 2019, Wiley. d Flexible large-area broadband photodetector from synthesised single-crystal $\mathrm{In}_{2} \mathrm{Se}_{3}$. Adapted with permission from Ref. [177]. Copyright 2019, American Association for the Advancement of Science. e Optical image of FET array of GaS from LM synthesis. Inset shows a single FET device. The scale bars on image and its corresponding inset are $500 \mu \mathrm{m}$ and $20 \mu \mathrm{m}$, respectively. Adapted with permission from Ref. [13]. Copyright 2017, Nature Publishing Group. f Atomically thin $\mathrm{WSe}_{2}$ printed in large areas by PLD shows high uniformity for the fabrication of FET devices. Adapted with permission from Ref. [33]. Copyright 2018, Wiley. g Optical image of FET array of large-area synthesised WS 2 monolayer. Adapted with permission from Ref. [83]. Copyright 2015, Royal Society of Chemistry Publishing Group

higher than the melting point of substrate metal, which may limit the applicability to other 2D materials. Single-crystal
TMDCs such as $\mathrm{MoS}_{2}$ have been achieved by CVD on a molten glass as a substrate with lateral dimensions of more 
than half a millimetre featuring high performances [57]. CVD method enables the growth of single-crystal graphene in record-breaking dimensions of metre sizes using $\mathrm{Cu}$ (111) as a substrate [11]. Comparing to ME, the CVD method is more expensive, time-consuming as well as requires dedicated engineering and expertise. On the other hand, MBE methods are shown to be a suitable method for required high-quality large-area 2D materials such as topological insulators. Similar to CVD methods a recipe is needed for MBE synthesis of 2D materials with larger grain sizes. The most critical parameters in generating large grain size $2 \mathrm{D}$ materials using MBE methods are found to include precursor flux and substrate temperature [181]. MBE method, however, requires sophisticated instrumentation and is expensive to operate $[22,41]$. Few CVD grown 2D materials are reported to achieve performances comparable to that of $\mathrm{ME}$ and MBE grown materials [1, 3, 32]. MOCVD method has been known to produce uniform crystals in wafer-scale but with the drawback of smaller grain sizes than that of CVD [1]. Other methods such as PLD and ALD are both shown to offer wafer-scale synthesis with precise thickness control and uniformity, which possibly has a broad scope for investigation and many possible 2D materials which have not been previously achieved can be synthesised [33, 34]. Recent emerging methods enabling the large-area synthesis of novel 2D materials, including the low-temperature LMbased process are in their infancy, however, can potentially offer pathways to production of high-quality atomically thin materials [14, 182]. In producing large-area uniform 2D oxides, ME methods do not provide a universal synthesis method since a majority of oxides have non-layered crystal structures. Recently, CVD methods have been reported to produce large-area 2D oxides of $\mathrm{MoO}_{3}$ [183] and consequently, the reliable transfer techniques [184] have been invented to enable large-area optoelectronics and sensing applications using $\mathrm{MoO}_{3}$. LM seems to be a frontier in 2D oxide synthesis with uniform thicknesses [47]. However, LM methods lacking investigation and optimisation of the crystal domain sizes which requires to be the focus of investigations for future device integrations. Recent outcomes present promising advancements in CVD methods as a frontier technology resolving significant challenges including high device performances, minimum grain boundary formation, enhanced scalability and reliable transfer techniques, however, process costs and complexity remain as a challenge.
Large-area synthesis of 2D materials has substantial implications for industrial uptake which has evolved to a fast-developing field of science. The recent development in the field of quantum computing will push the materials science explorations to optimise high-quality and large-scale synthesis of 2D materials systems featuring topological states, superconductivity and spin polarizability sites. There is nonetheless a vast scope for enhancing current technologies and developing emerging synthetic techniques.

Acknowledgements A.A.H. thanks the financial support from "National Natural Science Foundation of China" (No. 51850410506). J. Z. O acknowledges the financial support of the Australian Research Council (DE160100715).

Open Access This article is licensed under a Creative Commons Attribution 4.0 International License, which permits use, sharing, adaptation, distribution and reproduction in any medium or format, as long as you give appropriate credit to the original author(s) and the source, provide a link to the Creative Commons licence, and indicate if changes were made. The images or other third party material in this article are included in the article's Creative Commons licence, unless indicated otherwise in a credit line to the material. If material is not included in the article's Creative Commons licence and your intended use is not permitted by statutory regulation or exceeds the permitted use, you will need to obtain permission directly from the copyright holder. To view a copy of this licence, visit http://creativecommons.org/licenses/by/4.0/.

\section{References}

1. K. Kang, S. Xie, L. Huang, Y. Han, P.Y. Huang et al., Highmobility three-atom-thick semiconducting films with waferscale homogeneity. Nature 520, 656-660 (2015). https://doi. org/10.1038/nature14417

2. H. Lin, Q. Zhu, D. Shu, D. Lin, J. Xu et al., Growth of environmentally stable transition metal selenide films. Nat. Mater. 18(6), 602-607 (2019). https://doi.org/10.1038/s4156 3-019-0321-8

3. L. Zhou, K. Xu, A. Zubair, A.D. Liao, W. Fang et al., Largearea synthesis of high-quality uniform few-layer Mote ${ }_{2}$. J. Am. Chem. Soc. 137(37), 11892-11895 (2015). https://doi. org/10.1021/jacs.5b07452

4. Z. Guo, A. Wei, Y. Zhao, L. Tao, Y. Yang, Z. Zheng, D. Luo, J. Liu, J. Li, Controllable growth of large-area atomically thin $\mathrm{res}_{2}$ films and their thickness-dependent optoelectronic properties. Appl. Phys. Lett. 114(15), 153102 (2019). https ://doi.org/10.1063/1.5087456

5. J. Li, S. Cheng, Z. Liu, W. Zhang, H. Chang, Centimeterscale, large-area, few-layer $1 \mathrm{t}^{\prime}-\mathrm{WTe}_{2}$ films by chemical vapor deposition and its long-term stability in ambient condition. J. Phys. Chem. C 122(12), 7005-7012 (2018). https://doi. org/10.1021/acs.jpcc.8b00679 
6. Y.-H. Lee, X.-Q. Zhang, W. Zhang, M.-T. Chang, C.-T. Lin et al., Synthesis of large-area $\mathrm{Mos}_{2}$ atomic layers with chemical vapor deposition. Adv. Mater. 24(17), 2320-2325 (2012). https://doi.org/10.1002/adma.201104798

7. L. Jiao, W. Jie, Z. Yang, Y. Wang, Z. Chen et al., Layerdependent photoresponse of $2 \mathrm{D} \mathrm{MoS}_{2}$ films prepared by pulsed laser deposition. J. Mater. Chem. C 7(9), 2522-2529 (2019). https://doi.org/10.1039/C8TC04612C

8. H. Schmalzried, F.A. Kröger, The chemistry of imperfect crystals. Ber. Bunsenges. Phys. Chem. 68(6), 608 (1964). https://doi.org/10.1002/bbpc.19640680615

9. F. Kroger, F. Stieltjes, H. Vink, Thermodynamics and formulation of reactions involving imperfections in solids. Philips Res. Rep. 14, 557-601 (1959)

10. K.S. Novoselov, A.K. Geim, S.V. Morozov, D. Jiang, Y. Zhang, S.V. Dubonos, I.V. Grigorieva, A.A. Firsov, Electric field effect in atomically thin carbon films. Science 306(5696), 666-669 (2004). https://doi.org/10.1126/scien ce. 1102896

11. X. Xu, Z. Zhang, J. Dong, D. Yi, J. Niu et al., Ultrafast Epitaxial growth of metre-sized single-crystal graphene on industrial Cu foil. Sci. Bull. 62(15), 1074-1080 (2017). https ://doi.org/10.1016/j.scib.2017.07.005

12. C. Zhang, B. Anasori, A. Seral-Ascaso, S.-H. Park, N. McEvoy et al., Transparent, flexible, and conductive 2D titanium carbide (MXene) films with high volumetric capacitance. Adv. Mater. 29(36), 1702678 (2017). https://doi.org/10.1002/ adma.201702678

13. B.J. Carey, J.Z. Ou, R.M. Clark, K.J. Berean, A. Zavabeti et al., Wafer-scale two-dimensional semiconductors from printed oxide skin of liquid metals. Nat. Commun. 8, 14482 (2017). https://doi.org/10.1038/ncomms 14482

14. A. Zavabeti, J.Z. Ou, B.J. Carey, N. Syed, R. Orrell-Trigg et al., A liquid metal reaction environment for the room-temperature synthesis of atomically thin metal oxides. Science 358(6361), 332-335 (2017). https://doi.org/10.1126/scien ce.aao4249

15. K.A. Messalea, B.J. Carey, A. Jannat, N. Syed, M. Mohiuddin et al., $\mathrm{Bi}_{2} \mathrm{O}_{3}$ monolayers from elemental liquid bismuth. Nanoscale 10(33), 15615-15623 (2018). https://doi. org/10.1039/C8NR03788D

16. M.M. Alsaif, S. Kuriakose, S. Walia, N. Syed, A. Jannat et al., 2D SnO/ $/ \mathrm{In}_{2} \mathrm{O}_{3}$ van der waals heterostructure photodetector based on printed oxide skin of liquid metals. Adv. Mater. Interfaces 6(7), 1900007 (2019). https://doi.org/10.1002/ admi.201900007

17. L. Wang, X. Xu, L. Zhang, R. Qiao, M. Wu et al., Epitaxial growth of a 100-square-centimetre single-crystal hexagonal boron nitride monolayer on copper. Nature 570(7759), 91-95 (2019). https://doi.org/10.1038/s41586-019-1226-z

18. H. Cun, A. Hemmi, E. Miniussi, C. Bernard, B. Probst et al., Centimeter-sized single-orientation monolayer hexagonal boron nitride with or without nanovoids. Nano Lett. 18(2), 1205-1212 (2018). https://doi.org/10.1021/acs.nanol ett. $7 \mathrm{~b} 04752$
19. Y.-C. Lin, W. Zhang, J.-K. Huang, K.-K. Liu, Y.-H. Lee, C.-T. Liang, C.-W. Chu, L.-J. Li, Wafer-scale $\mathrm{Mos}_{2}$ thin layers prepared by $\mathrm{MoO}_{3}$ sulfurization. Nanoscale 4(20), 6637-6641 (2012). https://doi.org/10.1039/C2NR31833D

20. Y. Huang, E. Sutter, N.N. Shi, J. Zheng, T. Yang et al., Reliable exfoliation of large-area high-quality flakes of graphene and other two-dimensional materials. ACS Nano 9(11), 10612-10620 (2015). https://doi.org/10.1021/acsnano.5b042 58

21. L. Guan, B. Xing, X. Niu, D. Wang, Y. Yu et al., Metalassisted exfoliation of few-layer black phosphorus with high yield. Chem. Commun. 54(6), 595-598 (2018). https://doi. org/10.1039/C7CC08488A

22. E.P. Young, J. Park, T. Bai, C. Choi, R.H. DeBlock et al., Wafer-scale black arsenic-phosphorus thin-film synthesis validated with density functional perturbation theory predictions. ACS Appl. Nano Mater. 1(9), 4737-4745 (2018). https ://doi.org/10.1021/acsanm.8b00951

23. J. Shim, S.-H. Bae, W. Kong, D. Lee, K. Qiao et al., Controlled crack propagation for atomic precision handling of wafer-scale two-dimensional materials. Science 362(6415), 665-670 (2018). https://doi.org/10.1126/science.aat8126

24. S.B. Desai, S.R. Madhvapathy, M. Amani, D. Kiriya, M. Hettick et al., Gold-mediated exfoliation of ultralarge optoelectronically-perfect monolayers. Adv. Mater. 28(21), 40534058 (2016). https://doi.org/10.1002/adma.201506171

25. J. Lee, S. Pak, P. Giraud, Y.-W. Lee, Y. Cho et al., Thermodynamically stable synthesis of large-scale and highly crystalline transition metal dichalcogenide monolayers and their unipolar $\mathrm{n}-\mathrm{n}$ heterojunction devices. Adv. Mater. 29(33), 1702206 (2017). https://doi.org/10.1002/adma.201702206

26. C. Lan, Z. Zhou, Z. Zhou, C. Li, L. Shu et al., Wafer-scale synthesis of monolayer $\mathrm{WS}_{2}$ for high-performance flexible photodetectors by enhanced chemical vapor deposition. Nano Res. 11(6), 3371-3384 (2018). https://doi.org/10.1007/s 1227 4-017-1941-4

27. J.-K. Huang, J. Pu, C.-L. Hsu, M.-H. Chiu, Z.-Y. Juang et al., Large-area synthesis of highly crystalline $\mathrm{Wse}_{2}$ monolayers and device applications. ACS Nano 8(1), 923-930 (2014). https://doi.org/10.1021/nn405719x

28. L.-H. Zeng, D. Wu, S.-H. Lin, C. Xie, H.-Y. Yuan et al., Controlled synthesis of 2D palladium diselenide for sensitive photodetector applications. Adv. Funct. Mater. 29(1), 1806878 (2019). https://doi.org/10.1002/adfm.201806878

29. C. Cong, J. Shang, X. Wu, B. Cao, N. Peimyoo, C. Qiu, L. Sun, T. Yu, Synthesis and optical properties of large-Area single-crystalline $2 \mathrm{D}$ semiconductor $\mathrm{WS}_{2}$ monolayer from chemical vapor deposition. Adv. Opt. Mater. 2(2), 131-136 (2014). https://doi.org/10.1002/adom.201300428

30. S. Li, S. Wang, D.-M. Tang, W. Zhao, H. Xu et al., Halideassisted atmospheric pressure growth of large $\mathrm{WSe}_{2}$ and $\mathrm{WS}_{2}$ monolayer crystals. Appl. Mater. Today 1(1), 60-66 (2015). https://doi.org/10.1016/j.apmt.2015.09.001

31. H. Wang, Y. Chen, M. Duchamp, Q. Zeng, X. Wang et al., Large-area atomic layers of the charge-density-wave 
conductor TiSe ${ }_{2}$. Adv. Mater. 30(8), 1704382 (2018). https ://doi.org/10.1002/adma.201704382

32. J. Shi, X. Chen, L. Zhao, Y. Gong, M. Hong et al., Chemical vapor deposition grown wafer-scale $2 \mathrm{D}$ tantalum diselenide with robust charge-density-wave order. Adv. Mater. 30(44), 1804616 (2018). https://doi.org/10.1002/adma.201804616

33. S. Seo, H. Choi, S.-Y. Kim, J. Lee, K. Kim, S. Yoon, B.H. Lee, S. Lee, Growth of centimeter-scale monolayer and fewlayer $\mathrm{WSe}_{2}$ thin films on $\mathrm{SiO}_{2} / \mathrm{Si}$ substrate via pulsed laser deposition. Adv. Mater. Interfaces 5(20), 1800524 (2018). https://doi.org/10.1002/admi.201800524

34. B.D. Keller, A. Bertuch, J. Provine, G. Sundaram, N. Ferralis, J.C. Grossman, Process control of atomic layer deposition molybdenum oxide nucleation and sulfidation to large-area $\mathrm{MoS}_{2}$ monolayers. Chem. Mater. 29(5), 2024-2032 (2017). https://doi.org/10.1021/acs.chemmater.6b03951

35. J.S. Lee, S.H. Choi, S.J. Yun, Y.I. Kim, S. Boandoh et al., Wafer-scale single-crystal hexagonal boron nitride film via self-collimated grain formation. Science 362(6416), 817-821 (2018). https://doi.org/10.1126/science.aau2132

36. L. Song, L. Ci, H. Lu, P.B. Sorokin, C. Jin et al., Large scale growth and characterization of atomic hexagonal boron nitride layers. Nano Lett. 10(8), 3209-3215 (2010). https:// doi.org/10.1021/nl1022139

37. H. Jeong, D.Y. Kim, J. Kim, S. Moon, N. Han et al., Waferscale and selective-area growth of high-quality hexagonal boron nitride on $\mathrm{Ni}(111)$ by metal-organic chemical vapor deposition. Sci. Rep. 9(1), 5736 (2019). https://doi. org/10.1038/s41598-019-42236-4

38. C. Li, Y. Wu, B. Deng, Y. Xie, Q. Guo et al., Synthesis of crystalline black phosphorus thin film on sapphire. Adv. Mater. 30(6), 1703748 (2018). https://doi.org/10.1002/ adma. 201703748

39. L. Tao, E. Cinquanta, D. Chiappe, C. Grazianetti, M. Fanciulli, M. Dubey, A. Molle, D. Akinwande, Silicene field-effect transistors operating at room temperature. Nat. Nanotechnol. 10, 227 (2015). https://doi.org/10.1038/nnano.2014.325

40. Y. Wang, G. Qiu, R. Wang, S. Huang, Q. Wang et al., Fieldeffect transistors made from solution-grown two-dimensional tellurene. Nat. Electron. 1(4), 228-236 (2018). https://doi. org/10.1038/s41928-018-0058-4

41. R. Wu, I.K. Drozdov, S. Eltinge, P. Zahl, S. Ismail-Beigi, I. Božović, A. Gozar, Large-area single-crystal sheets of borophene on $\mathrm{Cu}(111)$ surfaces. Nat. Nanotechnol. 14(1), 44-49 (2019). https://doi.org/10.1038/s41565-018-0317-6

42. R. Wu, A. Gozar, I. Božović, Large-area borophene sheets on sacrificial $\mathrm{Cu}(111)$ films promoted by recrystallization from subsurface boron. NPJ Quantum. Mater. 4(1), 40 (2019). https ://doi.org/10.1038/s41535-019-0181-0

43. N. Bansal, N. Koirala, M. Brahlek, M.-G. Han, Y. Zhu et al., Robust topological surface states of $\mathrm{Bi}_{2} \mathrm{Se}_{3}$ thin films on amorphous $\mathrm{SiO}_{2} / \mathrm{Si}$ substrate and a large ambipolar gating effect. Appl. Phys. Lett. 104(24), 241606 (2014). https://doi. org/10.1063/1.4884348

44. C.-Z. Chang, J. Zhang, X. Feng, J. Shen, Z. Zhang et al., Experimental observation of the quantum anomalous hall effect in a magnetic topological insulator. Science 340(6129), 167-170 (2013). https://doi.org/10.1126/science.1234414

45. J. Wu, H. Yuan, M. Meng, C. Chen, Y. Sun et al., High electron mobility and quantum oscillations in non-encapsulated ultrathin semiconducting $\mathrm{Bi}_{2} \mathrm{O}_{2} \mathrm{Se}$. Nat. Nanotechnol. 12, 530 (2017). https://doi.org/10.1038/nnano.2017.43

46. W. Zeng, J. Li, L. Feng, H. Pan, X. Zhang, H. Sun, Z. Liu, Synthesis of large-area atomically thin bioi crystals with highly sensitive and controllable photodetection. Adv. Funct. Mater. 29(16), 1900129 (2019). https://doi.org/10.1002/ adfm.201900129

47. S. Li, M. Tian, Q. Gao, M. Wang, T. Li, Q. Hu, X. Li, Y. Wu, Nanometre-thin indium tin oxide for advanced high-performance electronics. Nat. Mater. 18(10), 1091-1097 (2019). https://doi.org/10.1038/s41563-019-0455-8

48. F. Wang, Y. Yu, X. Yin, P. Tian, X. Wang, Wafer-scale synthesis of ultrathin $\mathrm{CoO}$ nanosheets with enhanced electrochemical catalytic properties. J. Mater. Chem. A 5(19), 9060-9066 (2017). https://doi.org/10.1039/C7TA01857F

49. F. Wang, J.-H. Seo, G. Luo, M.B. Starr, Z. Li, D. Morgan et al., Nanometre-thick single-crystalline nanosheets grown at the water-air interface. Nat. Commun. 7, 10444 (2016). https://doi.org/10.1038/ncomms10444

50. N. Syed, A. Zavabeti, J.Z. Ou, M. Mohiuddin, N. Pillai et al., Printing two-dimensional gallium phosphate out of liquid metal. Nat. Commun. 9(1), 3618 (2018). https://doi. org/10.1038/s41467-018-06124-1

51. N. Syed, A. Zavabeti, K.A. Messalea, E. Della Gaspera, A. Elbourne et al., Wafer-sized ultrathin gallium and indium nitride nanosheets through the ammonolysis of liquid metal derived oxides. J. Am. Chem. Soc. 141(1), 104-108 (2019). https://doi.org/10.1021/jacs.8b11483

52. M.M.Y.A. Alsaif, N. Pillai, S. Kuriakose, S. Walia et al., Atomically thin $\mathrm{Ga}_{2} \mathrm{~S}_{3}$ from skin of liquid metals for electrical, optical and sensing applications. ACS Appl. Nano Mater. 2(7), 4665-4672 (2019). https://doi.org/10.1021/acsan m.9b01133

53. T. Wu, X. Zhang, Q. Yuan, J. Xue, G. Lu et al., Fast growth of inch-sized single-crystalline graphene from a controlled single nucleus on $\mathrm{Cu}-\mathrm{Ni}$ alloys. Nat. Mater. 15, 43 (2015). https://doi.org/10.1038/nmat4477

54. T. Chen, Y. Zhou, Y. Sheng, X. Wang, S. Zhou, J.H. Warner, Hydrogen-assisted growth of large-area continuous films of $\mathrm{MoS}_{2}$ on monolayer graphene. ACS Appl. Mater. Interfaces. 10(8), 7304-7314 (2018). https://doi.org/10.1021/acsam i. 7 b 14860

55. S. Najmaei, Z. Liu, W. Zhou, X. Zou, G. Shi et al., Vapour phase growth and grain boundary structure of molybdenum disulphide atomic layers. Nat. Mater. 12, 754 (2013). https:// doi.org/10.1038/nmat3673

56. W. Chen, J. Zhao, J. Zhang, L. Gu, Z. Yang et al., Oxygenassisted chemical vapor deposition growth of large singlecrystal and high-quality monolayer $\mathrm{MoS}_{2}$. J. Am. Chem. Soc. 137(50), 15632-15635 (2015). https://doi.org/10.1021/ jacs.5b10519 
57. Z. Zhang, X. Xu, J. Song, Q. Gao, S. Li, Q. Hu, X. Li, Y. $\mathrm{Wu}$, High-performance transistors based on monolayer CVD $\mathrm{MoS}_{2}$ grown on molten glass. Appl. Phys. Lett. 113(20), 202103 (2018). https://doi.org/10.1063/1.5051781

58. J. Chu, Y. Zhang, Y. Wen, R. Qiao, C. Wu et al., Sub-millimeter-scale growth of one-unit-cell-thick ferrimagnetic $\mathrm{Cr}_{2} \mathrm{~S}_{3}$ nanosheets. Nano Lett. 19(3), 2154-2161 (2019). https://doi. org/10.1021/acs.nanolett.9b00386

59. W. Yu, J. Li, T.S. Herng, Z. Wang, X. Zhao et al., Chemically exfoliated $\mathrm{VSe}_{2}$ monolayers with room-temperature ferromagnetism. Adv. Mater. 31(40), 1903779 (2019). https:// doi.org/10.1002/adma.201903779

60. K.S. Novoselov, D. Jiang, F. Schedin, T.J. Booth, V.V. Khotkevich, S.V. Morozov, A.K. Geim, Two-dimensional atomic crystals. Proc. Natl. Acad. Sci. USA 102(30), 10451-10453 (2005). https://doi.org/10.1073/pnas.05028 48102

61. K.-K. Liu, W. Zhang, Y.-H. Lee, Y.-C. Lin, M.-T. Chang et al., Growth of large-area and highly crystalline $\mathrm{MoS}_{2}$ thin layers on insulating substrates. Nano Lett. 12(3), 1538-1544 (2012). https://doi.org/10.1021/nl2043612

62. L. Tao, K. Chen, Z. Chen, W. Chen, X. Gui, H. Chen, X. Li, J.-B. Xu, Centimeter-scale cvd growth of highly crystalline single-layer $\mathrm{MoS}_{2}$ film with spatial homogeneity and the visualization of grain boundaries. ACS Appl. Mater. Interfaces 9(13), 12073-12081 (2017). https://doi.org/10.1021/acsam i. $7 b 00420$

63. K.S. Kim, Y. Zhao, H. Jang, S.Y. Lee, J.M. Kim et al., Largescale pattern growth of graphene films for stretchable transparent electrodes. Nature 457(7230), 706-710 (2009). https ://doi.org/10.1038/nature07719

64. X. Li, W. Cai, J. An, S. Kim, J. Nah et al., Large-area synthesis of high-quality and uniform graphene films on copper foils. Science 324(5932), 1312-1314 (2009). https://doi. org/10.1126/science.1171245

65. Y. Zhao, H. Lee, W. Choi, W. Fei, C.J. Lee, Large-area synthesis of monolayer $\mathrm{MoSe}_{2}$ films on $\mathrm{SiO}_{2} / \mathrm{Si}$ substrates by atmospheric pressure chemical vapor deposition. RSC Adv. 7(45), 27969-27973 (2017). https://doi.org/10.1039/C7RA0 $3642 \mathrm{~F}$

66. H. Tian, Y. He, P. Das, Z. Cui, W. Shi, A. Khanaki, R.K. Lake, J. Liu, Growth dynamics of millimeter-sized singlecrystal hexagonal boron nitride monolayers on secondary recrystallized Ni (100) substrates. Adv. Mater. Interfaces 6(22), 1901198 (2019). https://doi.org/10.1002/admi.20190 1198

67. Z. Xu, H. Tian, A. Khanaki, R. Zheng, M. Sujam, J. Liu, Large-area growth of multi-layer hexagonal boron nitride on polished cobalt foils by plasma-assisted molecular beam epitaxy. Sci. Rep. 7, 43100 (2017). https://doi.org/10.1038/ srep43100

68. M. Marx, S. Nordmann, J. Knoch, C. Franzen, C. Stampfer et al., Large-area $\mathrm{MoS}_{2}$ deposition via MOVPE. J. Cryst. Growth 464, 100-104 (2017). https://doi.org/10.1016/j.jcrys gro.2016.11.020
69. D. Andrzejewski, H. Myja, M. Heuken, A. Grundmann, H. Kalisch, A. Vescan, T. Kümmell, G. Bacher, Scalable largearea $\mathrm{p}-\mathrm{i}-\mathrm{n}$ light-emitting diodes based on $\mathrm{WS}_{2}$ monolayers grown via MOCVD. ACS Photonics 6(8), 1832-1839 (2019). https://doi.org/10.1021/acsphotonics.9b00311

70. H. Cun, M. Macha, H. Kim, K. Liu, Y. Zhao, T. LaGrange, A. Kis, A. Radenovic, Wafer-scale MOCVD growth of monolayer $\mathrm{MoS}_{2}$ on sapphire and $\mathrm{SiO}_{2}$. Nano Res. 12(10), 26462652 (2019). https://doi.org/10.1007/s12274-019-2502-9

71. A. Jannat, Q. Yao, A. Zavabeti, N. Syed, B.Y. Zhang et al., Ordered-vacancy-enabled indium sulphide printed in waferscale with enhanced electron mobility. Mater. Horiz. (2019). https://doi.org/10.1039/C9MH01365B

72. R. Ma, T. Sasaki, Two-dimensional oxide and hydroxide nanosheets: controllable high-quality exfoliation, molecular assembly, and exploration of functionality. Acc. Chem. 48(1), 136-143 (2015). https://doi.org/10.1021/ar500311w

73. H. Tao, Y. Zhang, Y. Gao, Z. Sun, C. Yan, J. Texter, Scalable exfoliation and dispersion of two-dimensional materials-an update. Phys. Chem. Chem. Phys. 19(2), 921-960 (2017). https://doi.org/10.1039/C6CP06813H

74. X. Cai, Y. Luo, B. Liu, H.-M. Cheng, Preparation of 2D material dispersions and their applications. Chem. Soc. Rev. 47(16), 6224-6266 (2018). https://doi.org/10.1039/ C8CS00254A

75. Z. Shi, Q. Li, R. Jiang, C. Zhang, W. Yin, T. Wu, X. Xie, Influence of oxygen on the synthesis of large area hexagonal boron nitride on $\mathrm{Fe}_{2} \mathrm{~B}$ substrate. Mater. Lett. 247, 52-55 (2019). https://doi.org/10.1016/j.matlet.2019.03.095

76. D. Geng, X. Zhao, K. Zhou, W. Fu, Z. Xu, S.J. Pennycook, L.K. Ang, H.Y. Yang, From self-assembly hierarchical $\mathrm{H}-\mathrm{Bn}$ patterns to centimeter-scale uniform monolayer $\mathrm{H}-\mathrm{bn}$ film. Adv. Mater. Interfaces 6(1), 1801493 (2019). https:// doi.org/10.1002/admi.201801493

77. F. Hui, M.A. Villena, W. Fang, A.-Y. Lu, J. Kong et al., Synthesis of large-area multilayer hexagonal boron nitride sheets on iron substrates and its use in resistive switching devices. 2D Mater. 5(3), 031011 (2018). https://doi. org/10.1088/20531583/aac615

78. J. Zhou, J. Lin, X. Huang, Y. Zhou, Y. Chen et al., A library of atomically thin metal chalcogenides. Nature 556(7701), 355-359 (2018). https://doi.org/10.1038/s4158 6-018-0008-3

79. Z. Hu, Z. Wu, C. Han, J. He, Z. Ni, W. Chen, Two-dimensional transition metal dichalcogenides: interface and defect engineering. Chem. Soc. Rev. 47(9), 3100-3128 (2018). https ://doi.org/10.1039/C8CS00024G

80. P. Masih Das, J.P. Thiruraman, Y.-C. Chou, G. Danda, M. Drndić, Centimeter-scale nanoporous 2D membranes and Ion transport: porous $\mathrm{Mos}_{2}$ monolayers in a few-layer matrix. Nano Lett. 19(1), 392-399 (2019). https://doi.org/10.1021/ acs.nanolett.8b04155

81. P. Liu, T. Luo, J. Xing, H. Xu, H. Hao, H. Liu, J. Dong, Large-area $\mathrm{Ws}_{2}$ film with big single domains grown by chemical vapor deposition. Nanoscale Res. Lett. 12(1), 558 (2017). https://doi.org/10.1186/s11671-017-2329-9 
82. S.J. Yun, S.H. Chae, H. Kim, J.C. Park, J.-H. Park et al., Synthesis of centimeter-scale monolayer tungsten disulfide film on gold foils. ACS Nano 9(5), 5510-5519 (2015). https://doi. org/10.1021/acsnano.5b01529

83. C. Lan, C. Li, Y. Yin, Y. Liu, Large-area synthesis of monolayer $\mathrm{Ws}_{2}$ and its ambient-sensitive photo-detecting performance. Nanoscale 7(14), 5974-5980 (2015). https://doi. org/10.1039/C5NR01205H

84. S. Wagner, C. Yim, N. McEvoy, S. Kataria, V. Yokaribas et al., Highly sensitive electromechanical piezoresistive pressure sensors based on large-area layered $\mathrm{PtSe}_{2}$ films. Nano Lett. 18(6), 3738-3745 (2018). https://doi.org/10.1021/acs. nanolett.8b00928

85. M. Velický, G.E. Donnelly, W.R. Hendren, S. McFarland, D. Scullion et al., Mechanism of gold-assisted exfoliation of centimeter-sized transition-metal dichalcogenide monolayers. ACS Nano 12(10), 10463-10472 (2018). https:// doi.org/10.1021/acsnano.8b06101

86. H.M. Gramling, C.M. Towle, S.B. Desai, H. Sun, E.C. Lewis et al., Spatially precise transfer of patterned monolayer $\mathrm{WS}_{2}$ and $\mathrm{MoS}_{2}$ with features larger than $10^{4} \mu \mathrm{m}^{2}$ directly from multilayer sources. ACS Appl. Electron. Mater. 1(3), 407-416 (2019). https://doi.org/10.1021/acsae $1 \mathrm{~m} .8 \mathrm{~b} 00128$

87. J. Peng, J. Wu, X. Li, Y. Zhou, Z. Yu et al., Very Largesized transition metal dichalcogenides monolayers from fast exfoliation by manual shaking. J. Am. Chem. Soc. 139(26), 9019-9025 (2017). https://doi.org/10.1021/jacs.7b04332

88. Q. Zhang, J. Lu, Z. Wang, Z. Dai, Y. Zhang et al., Reliable synthesis of large-area monolayer $\mathrm{WS}_{2}$ single crystals, films, and heterostructures with extraordinary photoluminescence induced by water intercalation. Adv. Opt. Mater. 6(12), 1701347 (2018). https://doi.org/10.1002/adom.201701347

89. Z. Zhao, D. Wu, J. Guo, E. Wu, C. Jia et al., Synthesis of large-area $2 \mathrm{D} \mathrm{WS}_{2}$ films and fabrication of a heterostructure for self-powered ultraviolet photodetection and imaging applications. J. Mater. Chem. C 7(39), 12121-12126 (2019). https://doi.org/10.1039/C9TC03866C

90. A.B. Maghirang, Z.-Q. Huang, R.A.B. Villaos, C.-H. Hsu, L.-Y. Feng et al., Predicting two-dimensional topological phases in janus materials by substitutional doping in transition metal dichalcogenide monolayers. NPJ 2D Mater. Appl. 3(1), 35 (2019). https://doi.org/10.1038/s41699-019-0118-2

91. K.K. Kim, H.S. Lee, Y.H. Lee, Synthesis of hexagonal boron nitride heterostructures for 2D van der waals electronics. Chem. Soc. Rev. 47(16), 6342-6369 (2018). https://doi. org/10.1039/C8CS00450A

92. X.B. Ren, J.C. Dong, P. Yang, J.D. Li, G.Y. Lu et al., Grain boundaries in chemical-vapor-deposited atomically thin hexagonal boron nitride. Phys. Rev. Mater. 3(1), 014004 (2019). https://doi.org/10.1103/PhysRevMaterials.3.014004

93. G. Lu, T. Wu, Q. Yuan, H. Wang, H. Wang, F. Ding, X. Xie, M. Jiang, Synthesis of large single-crystal hexagonal boron nitride grains on $\mathrm{Cu}-\mathrm{Ni}$ alloy. Nat. Commun. 6, 6160 (2015). https://doi.org/10.1038/ncomms7160
94. B.C. Bayer, S. Caneva, T.J. Pennycook, J. Kotakoski, C. Mangler, S. Hofmann, J.C. Meyer, Introducing overlapping grain boundaries in chemical vapor deposited hexagonal boron nitride monolayer films. ACS Nano 11(5), 4521-4527 (2017). https://doi.org/10.1021/acsnano.6b08315

95. X. Tong, K. Liu, M. Zeng, L. Fu, Vapor-phase growth of high-quality wafer-scale two-dimensional materials. InfoMat 1(4), 460-478 (2019). https://doi.org/10.1002/inf2.12038

96. S.M. Kim, A. Hsu, M.H. Park, S.H. Chae, S.J. Yun et al., Synthesis of large-area multilayer hexagonal boron nitride for high material performance. Nat. Commun. 6, 8662 (2015). https://doi.org/10.1038/ncomms9662

97. J. Xie, L. Liao, Y. Gong, Y. Li, F. Shi et al., Stitching H-Bn by atomic layer deposition of lif as a stable interface for lithium metal anode. Sci. Adv. 3(11), eaa3170 (2017). https://doi. org/10.1126/sciadv.aao3170

98. H. Park, T.K. Kim, S.W. Cho, H.S. Jang, S.I. Lee, S.-Y. Choi, Large-scale synthesis of uniform hexagonal boron nitride films by plasma-enhanced atomic layer deposition. Sci. Rep. 7, 40091 (2017). https://doi.org/10.1038/srep40091

99. A. Molle, J. Goldberger, M. Houssa, Y. Xu, S.-C. Zhang, D. Akinwande, Buckled two-dimensional Xene sheets. Nat. Mater. 16(2), 163-169 (2017). https://doi.org/10.1038/nmat4 802

100. F. Reis, G. Li, L. Dudy, M. Bauernfeind, S. Glass, W. Hanke, R. Thomale, J. Schäfer, R. Claessen, Bismuthene on a sic substrate: a candidate for a high-temperature quantum spin hall material. Science 357(6348), 287-290 (2017). https:// doi.org/10.1126/science.aai8142

101. H.-S. Tsai, Y.-Z. Chen, H. Medina, T.-Y. Su, T.-S. Chou et al., Direct formation of large-scale multi-layered germanene on Si substrate. Phys. Chem. Chem. Phys. 17(33), 21389-21393 (2015). https://doi.org/10.1039/C5CP02469B

102. J. Yuhara, Y. Fujii, K. Nishino, N. Isobe, M. Nakatake, L. Xian, A. Rubio, G. Le Lay, Large area planar stanene epitaxially grown on $\operatorname{Ag}\left(\begin{array}{lll}1 & 1 & 1\end{array}\right)$. 2D Mater. 5(2), 025002 (2018). https://doi.org/10.1088/2053-1583/aa9ea0

103. J. Yuhara, B. He, N. Matsunami, M. Nakatake, G. Le Lay, Graphene's latest cousin: plumbene epitaxial growth on a "Nano Watercube". Adv. Mater. 31(27), 1901017 (2019). https://doi.org/10.1002/adma.201901017

104. E.S. Walker, S.R. Na, D. Jung, S.D. March, J.-S. Kim et al., Large-area dry transfer of single-crystalline epitaxial bismuth thin films. Nano Lett. 16(11), 6931-6938 (2016). https://doi. org/10.1021/acs.nanolett.6b02931

105. J. Zhao, H. Liu, Z. Yu, R. Quhe, S. Zhou et al., Rise of silicene: a competitive 2D material. Prog. Mater Sci. 83, 24-151 (2016). https://doi.org/10.1016/j.pmats ci.2016.04.001

106. A.J. Mannix, X.-F. Zhou, B. Kiraly, J.D. Wood, D. Alducin et al., Synthesis of borophenes: anisotropic, two-dimensional boron polymorphs. Science 350(6267), 1513-1516 (2015). https://doi.org/10.1126/science.aad1080

107. B. Feng, J. Zhang, Q. Zhong, W. Li, S. Li, H. Li, P. Cheng, S. Meng, L. Chen, K. Wu, Experimental realization of 
two-dimensional boron sheets. Nat. Chem. 8, 563 (2016). https://doi.org/10.1038/nchem.2491

108. Z. Yang, Z. Wu, Y. Lyu, J. Hao, Centimeter-scale growth of two-dimensional layered high-mobility bismuth films by pulsed laser deposition. InfoMat 1(1), 98-107 (2019). https ://doi.org/10.1002/inf2.12001

109. S.K. Pradhan, R. Barik, Observation of the magneto-transport property in a millimeter-long topological insulator $\mathrm{Bi}_{2} \mathrm{Te}_{3}$ thin-film hall bar device. Appl. Mater. Today 7, 55-59 (2017). https://doi.org/10.1016/j.apmt.2017.02.002

110. J. Krumrain, G. Mussler, S. Borisova, T. Stoica, L. Plucinski, C.M. Schneider, D. Grützmacher, MBe growth optimization of topological insulator $\mathrm{Bi}_{2} \mathrm{Te}_{3}$ films. J. Cryst. Growth 324(1), 115-118 (2011). https://doi.org/10.1016/j.jcrys gro.2011.03.008

111. J.E. Brom, Growth and Characterization of Bismuth Selenide Thin Films by Chemical Vapor Deposition. Ph.D. Thesis (2014)

112. C.-M. Hyun, J.-H. Choi, S.W. Lee, S.-Y. Seo, M.-J. Lee, S.-H. Kwon, J.-H. Ahn, Synthesis of $\mathrm{Bi}_{2} \mathrm{Te}_{3}$ single crystals with lateral size up to tens of micrometers by vapor transport and its potential for thermoelectric applications. Cryst. Growth Des. 19(4), 2024-2029 (2019). https://doi. org/10.1021/acs.cgd.8b01931

113. X. Li, F. Cui, Q. Feng, G. Wang, X. Xu et al., Controlled growth of large-area anisotropic $\mathrm{ReS}_{2}$ atomic layer and its photodetector application. Nanoscale 8(45), 18956-18962 (2016). https://doi.org/10.1039/C6NR07233J

114. F. Massoth, D. Scarpiello, Kinetics of bismuth oxide reduction with propylene. J. Catal. 21(2), 225-238 (1971). https ://doi.org/10.1016/0021-9517(71)90141-2

115. B. Trawiński, B. Bochentyn, B. Kusz, A Study of a reduction of a micro- and nanometric bismuth oxide in hydrogen atmosphere. Thermochim. Acta 669, 99-108 (2018). https ://doi.org/10.1016/j.tca.2018.09.010

116. Y. Dou, L. Zhang, X. Xu, Z. Sun, T. Liao, S.X. Dou, Atomically thin non-layered nanomaterials for energy storage and conversion. Chem. Soc. Rev. 46(23), 7338-7373 (2017). https://doi.org/10.1039/C7CS00418D

117. C. Tan, H. Zhang, Wet-chemical synthesis and applications of non-layer structured two-dimensional nanomaterials. Nat. Commun. 6, 7873 (2015). https://doi.org/10.1038/ ncomms 8873

118. W. Yang, X. Zhang, Y. Xie, Advances and challenges in chemistry of two-dimensional nanosheets. Nano Today 11(6), 793-816 (2016). https://doi.org/10.1016/j.nanto d.2016.10.004

119. T. Maluangnont, K. Matsuba, F. Geng, R. Ma, Y. Yamauchi, T. Sasaki, Osmotic swelling of layered compounds as a route to producing high-quality two-dimensional materials. A comparative study of tetramethylammonium versus tetrabutylammonium cation in a lepidocrocite-type titanate. Chem. Mater. 25(15), 3137-3146 (2013). https://doi. org/10.1021/cm401409s

120. X. Huang, S. Li, Y. Huang, S. Wu, X. Zhou et al., Synthesis of hexagonal close-packed gold nanostructures. Nat.
Commun. 2, 292 (2011). https://doi.org/10.1038/ncomm s1291

121. S. Fullam, D. Cottell, H. Rensmo, D. Fitzmaurice, Carbon nanotube templated self-assembly and thermal processing of gold nanowires. Adv. Mater. 12(19), 1430-1432 (2000). https://doi.org/10.1002/1521-4095(200010)12:19\%3c143 0:AID-ADMA1430\%3e3.0.CO;2-8

122. T. Li, H. Jin, Z. Liang, L. Huang, Y. Lu et al., Synthesis of single crystalline two-dimensional transition-metal phosphides via a salt-templating method. Nanoscale 10(15), 6844-6849 (2018). https://doi.org/10.1039/C8NR01556B

123. C. Feng, J. Zhang, Y. He, C. Zhong, W. Hu, L. Liu, Y. Deng, Sub-3 Nm Co $\mathrm{O}_{4}$ nanofilms with enhanced supercapacitor properties. ACS Nano 9(2), 1730-1739 (2015). https://doi.org/10.1021/nn506548d

124. K. Kalantar-zadeh, J.Z. Ou, T. Daeneke, A. Mitchell, T. Sasaki, M.S. Fuhrer, Two dimensional and layered transition metal oxides. Appl. Mater. Today 5, 73-89 (2016). https:// doi.org/10.1016/j.apmt.2016.09.012

125. L. Qin, B. Kattel, T.R. Kafle, M. Alamri, M. Gong et al., Scalable graphene-on-organometal halide perovskite heterostructure fabricated by dry transfer. Adv. Mater. Interfaces 6(1), 1801419 (2019). https://doi.org/10.1002/admi.20180 1419

126. L. Yu, Y.-H. Lee, X. Ling, E.J.G. Santos, Y.C. Shin et al., Graphene/ $\mathrm{MoS}_{2}$ hybrid technology for large-scale twodimensional electronics. Nano Lett. 14(6), 3055-3063 (2014). https://doi.org/10.1021/nl404795z

127. I.V. Vlassiouk, Y. Stehle, P.R. Pudasaini, R.R. Unocic, P.D. Rack et al., Evolutionary selection growth of two-dimensional materials on polycrystalline substrates. Nat. Mater. 17(4), 318-322 (2018). https://doi.org/10.1038/s41563-018-0019-3

128. A. Van der Drift, Evolutionary selection, a principle governing growth orientation in vapour-deposited layers. Philips Res. Rep. 22(3), 267 (1967)

129. X. Xue, Q. Xu, H. Wang, S. Liu, Q. Jiang et al., Gas-flowdriven aligned growth of graphene on liquid copper. Chem. Mater. 31(4), 1231-1236 (2019). https://doi.org/10.1021/acs. chemmater.8b03998

130. X. Sun, L. Lin, L. Sun, J. Zhang, D. Rui et al., Low-temperature and rapid growth of large single-crystalline graphene with ethane. Small 14(3), 1702916 (2018). https://doi. org/10.1002/smll.201702916

131. A. Koh, Y. Foong, D.H. Chua, Cooling rate and energy dependence of pulsed laser fabricated graphene on nickel at reduced temperature. Appl. Phys. Lett. 97(11), 114102 (2010). https://doi.org/10.1063/1.3489993

132. A. Shivayogimath, P.R. Whelan, D.M.A. Mackenzie, B. Luo, D. Huang et al., Do-it-yourself transfer of large-area graphene using an office laminator and water. Chem. Mater. 31(7), 2328-2336 (2019). https://doi.org/10.1021/acs.chemm ater.8b04196

133. J. Wang, C. Teng, Y. Jiang, Y. Zhu, L. Jiang, Wetting-induced climbing for transferring interfacially assembled large-area ultrathin pristine graphene film. Adv. Mater. 31(10), 1806742 (2019). https://doi.org/10.1002/adma.201806742 
134. A. Karmakar, F. Vandrevala, F. Gollier, M.A. Philip, S. Shahi, E. Einarsson, Approaching completely continuous centimeter-scale graphene by copolymer-assisted transfer. RSC Adv. 8(4), 1725-1729 (2018). https://doi.org/10.1039/C7RA1 $2328 \mathrm{~K}$

135. T. Choi, S.J. Kim, S. Park, T. Hwang, Y. Jeon, B.H. Hong, 2015 IEEE Int. Electron Dev. Meet. (IEDM). 27. 21-27.24 (2015). https://doi.org/10.1109/iedm.2015.7409784

136. Z. Huang, A. Zhou, J. Wu, Y. Chen, X. Lan, H. Bai, L. Li, Bottom-up preparation of ultrathin $2 \mathrm{D}$ aluminum oxide nanosheets by duplicating graphene oxide. Adv. Mater. 28(8), 1703-1708 (2016). https://doi.org/10.1002/adma.201504484

137. Z. Lin, B.R. Carvalho, E. Kahn, R. Lv, R. Rao, H. Terrones, M.A. Pimenta, M. Terrones, Defect engineering of two-dimensional transition metal dichalcogenides. 2D Mater. 3(2), 022002 (2016). https://doi.org/10.1088/2053$1583 / 3 / 2 / 022002$

138. J. Li, W. Su, F. Chen, L. Fu, S. Ding, K. Song, X. Huang, L. Zhang, Atypical defect-mediated photoluminescence and resonance raman spectroscopy of monolayer $\mathrm{Ws}_{2}$. J. Phys. Chem. C 123(6), 3900-3907 (2019). https://doi. org/10.1021/acs.jpcc.8b11647

139. P. Vancsó, G.Z. Magda, J. Pető, J.-Y. Noh, Y.-S. Kim, C. Hwang, L.P. Biró, L. Tapasztó, The intrinsic defect structure of exfoliated $\mathrm{MoS}_{2}$ single layers revealed by scanning tunneling microscopy. Sci. Rep. 6, 29726 (2016). https:// doi.org/10.1038/srep29726

140. F. Cheng, Z. Ding, H. Xu, S.J.R. Tan, I. Abdelwahab, J. $\mathrm{Su}$, P. Zhou, J. Martin, K.P. Loh, Epitaxial growth of single-layer niobium selenides with controlled stoichiometric phases. Adv. Mater. Interfaces 5(15), 1800429 (2018). https ://doi.org/10.1002/admi.201800429

141. W. Zhou, X. Zou, S. Najmaei, Z. Liu, Y. Shi et al., Intrinsic structural defects in monolayer molybdenum disulfide. Nano Lett. 13(6), 2615-2622 (2013). https://doi. org/10.1021/nl4007479

142. P.K. Chow, E. Singh, B.C. Viana, J. Gao, J. Luo et al., Wetting of mono and few-layered $\mathrm{WS}_{2}$ and $\mathrm{MoS}_{2}$ films supported on $\mathrm{Si} / \mathrm{SiO}_{2}$ substrates. ACS Nano 9(3), 3023-3031 (2015). https://doi.org/10.1021/nn5072073

143. A.L. Elías, N. Perea-López, A. Castro-Beltrán, A. Berkdemir, R. Lv et al., Controlled synthesis and transfer of large-area $\mathrm{WS}_{2}$ sheets: from single layer to few layers. ACS Nano 7(6), 5235-5242 (2013). https://doi.org/10.1021/ $\mathrm{nn} 400971 \mathrm{k}$

144. N. Peimyoo, J. Shang, C. Cong, X. Shen, X. Wu, E.K.L. Yeow, T. Yu, Nonblinking, Intense two-dimensional light emitter: monolayer $\mathrm{WS}_{2}$ triangles. ACS Nano 7(12), 1098510994 (2013). https://doi.org/10.1021/nn4046002

145. I.S. Kim, V.K. Sangwan, D. Jariwala, J.D. Wood, S. Park et al., Influence of stoichiometry on the optical and electrical properties of chemical vapor deposition derived $\mathrm{MoS}_{2}$. ACS Nano 8(10), 10551-10558 (2014). https://doi.org/10.1021/ nn503988x

146. A.M. van der Zande, P.Y. Huang, D.A. Chenet, T.C. Berkelbach, Y. You et al., Grains and grain boundaries in highly crystalline monolayer molybdenum disulphide. Nat. Mater. 12, 554 (2013). https://doi.org/10.1038/nmat3633

147. R.G. Mendes, J. Pang, A. Bachmatiuk, H.Q. Ta, L. Zhao, T. Gemming, L. Fu, Z. Liu, M.H. Rümmeli, Electron-driven in situ transmission electron microscopy of 2D transition metal dichalcogenides and their 2D heterostructures. ACS Nano 13(2), 978-995 (2019). https://doi.org/10.1021/acsna no. 8 b08079

148. Y. Yin, J. Han, Y. Zhang, X. Zhang, P. Xu et al., Contributions of phase, sulfur vacancies, and edges to the hydrogen evolution reaction catalytic activity of porous molybdenum disulfide nanosheets. J. Am. Chem. Soc. 138(25), 7965-7972 (2016). https://doi.org/10.1021/jacs.6b03714

149. X. Ding, F. Peng, J. Zhou, W. Gong, G. Slaven, K.P. Loh, C.T. Lim, D.T. Leong, Defect engineered bioactive transition metals dichalcogenides quantum dots. Nat. Commun. 10(1), 41 (2019). https://doi.org/10.1038/s41467-018-07835-1

150. J. Xie, H. Zhang, S. Li, R. Wang, X. Sun et al., Defect-rich $\mathrm{MoS}_{2}$ ultrathin nanosheets with additional active edge sites for enhanced electrocatalytic hydrogen evolution. Adv. Mater. 25(40), 5807-5813 (2013). https://doi.org/10.1002/ adma.201302685

151. H.-P. Komsa, J. Kotakoski, S. Kurasch, O. Lehtinen, U. Kaiser, A.V. Krasheninnikov, Two-dimensional transition metal dichalcogenides under electron irradiation: defect production and doping. Phys. Rev. Lett. 109(3), 035503 (2012). https:// doi.org/10.1103/PhysRevLett.109.035503

152. Z. Liu, K. Suenaga, Z. Wang, Z. Shi, E. Okunishi, S. Iijima, Identification of active atomic defects in a monolayered tungsten disulphide nanoribbon. Nat. Commun. 2, 213 (2011). https://doi.org/10.1038/ncomms 1224

153. M.R. Islam, N. Kang, U. Bhanu, H.P. Paudel, M. Erementchouk, L. Tetard, M.N. Leuenberger, S.I. Khondaker, Tuning the electrical property via defect engineering of single layer $\mathrm{MoS}_{2}$ by oxygen plasma. Nanoscale 6(17), 1003310039 (2014). https://doi.org/10.1039/C4NR02142H

154. M. Yamamoto, S. Dutta, S. Aikawa, S. Nakaharai, K. Wakabayashi, M.S. Fuhrer, K. Ueno, K. Tsukagoshi, Self-limiting layer-by-layer oxidation of atomically thin $\mathrm{WSe}_{2}$. Nano Lett. 15(3), 2067-2073 (2015). https://doi.org/10.1021/nl5049753

155. M. Chen, H. Nam, S. Wi, G. Priessnitz, I.M. Gunawan, X. Liang, Multibit data storage states formed in plasma-treated $\mathrm{MoS}_{2}$ transistors. ACS Nano 8(4), 4023-4032 (2014). https ://doi.org/10.1021/nn501181t

156. T.-Y. Kim, K. Cho, W. Park, J. Park, Y. Song, S. Hong, W.-K. Hong, T. Lee, Irradiation effects of high-energy proton beams on $\mathrm{MoS}_{2}$ field effect transistors. ACS Nano 8(3), 2774-2781 (2014). https://doi.org/10.1021/nn4064924

157. S. Tongay, J. Suh, C. Ataca, W. Fan, A. Luce et al., Defects activated photoluminescence in two-dimensional semiconductors: interplay between bound, charged, and free excitons. Sci. Rep. 3, 2657 (2013). https://doi.org/10.1038/srep02657

158. J. Feng, K. Liu, M. Graf, M. Lihter, R.D. Bulushev et al., Electrochemical reaction in single layer $\mathrm{MoS}_{2}$ : nanopores opened atom by atom. Nano Lett. 15(5), 3431-3438 (2015). https://doi.org/10.1021/acs.nanolett.5b00768 
159. B. Groven, A. Nalin Mehta, H. Bender, J. Meersschaut, T. Nuytten et al., Two-dimensional crystal grain size tuning in $\mathrm{WS}_{2}$ atomic layer deposition: an insight in the nucleation mechanism. Chem. Mater. 30(21), 7648-7663 (2018). https ://doi.org/10.1021/acs.chemmater.8b02924

160. S. Feldmann, S. Macpherson, S.P. Senanayak, M. AbdiJalebi, J.P.H. Rivett et al., Photodoping through local charge carrier accumulation in alloyed hybrid perovskites for highly efficient luminescence. Nat. Photonics 10, 15-20 (2019). https://doi.org/10.1038/s41566-019-0546-8

161. C.-P. Lu, G. Li, J. Mao, L.-M. Wang, E.Y. Andrei, Bandgap, mid-gap states, and gating effects in $\mathrm{MoS}_{2}$. Nano Lett. 14(8), 4628-4633 (2014). https://doi.org/10.1021/n1501659n

162. H. Nan, Z. Wang, W. Wang, Z. Liang, Y. Lu et al., Strong photoluminescence enhancement of $\mathrm{MoS}_{2}$ through defect engineering and oxygen bonding. ACS Nano 8(6), 57385745 (2014). https://doi.org/10.1021/nn500532f

163. W. Zhu, T. Low, Y.-H. Lee, H. Wang, D.B. Farmer, J. Kong, F. Xia, P. Avouris, Electronic transport and device prospects of monolayer molybdenum disulphide grown by chemical vapour deposition. Nat. Commun. 5, 3087 (2014). https:// doi.org/10.1038/ncomms4087

164. A. McCreary, A. Berkdemir, J. Wang, M.A. Nguyen, A.L. Elías et al., Distinct Photoluminescence and raman spectroscopy signatures for identifying highly crystalline $\mathrm{WS}_{2}$ monolayers produced by different growth methods. J. Mater. Res. Technol. 31(7), 931-944 (2016). https://doi.org/10.1557/ jmr.2016.47

165. N. Kang, H.P. Paudel, M.N. Leuenberger, L. Tetard, S.I. Khondaker, Photoluminescence quenching in single-layer $\mathrm{MoS}_{2}$ via oxygen plasma treatment. J. Phys. Chem. C 118(36), 21258-21263 (2014). https://doi.org/10.1021/jp506 $964 \mathrm{~m}$

166. W. Shi, M.-L. Lin, Q.-H. Tan, X.-F. Qiao, J. Zhang, P.-H. Tan, Raman and photoluminescence spectra of two-dimensional nanocrystallites of monolayer $\mathrm{WS}_{2}$ and $\mathrm{WSe}_{2}$. 2D Mater. 3(2), 025016 (2016). https://doi.org/10.1088/2053-1583/3/2/02501 6

167. S. Yuan, R. Roldán, M.I. Katsnelson, F. Guinea, Effect of point defects on the optical and transport properties of $\mathrm{MoS}_{2}$ and $\mathrm{WS}_{2}$. Phys. Rev. B 90(4), 041402 (2014). https://doi. org/10.1103/PhysRevB.90.041402

168. Q. Ma, M. Isarraraz, C.S. Wang, E. Preciado, V. Klee et al., Postgrowth tuning of the bandgap of single-layer molybdenum disulfide films by sulfur/selenium exchange. ACS Nano 8(5), 4672-4677 (2014). https://doi.org/10.1021/nn5004327

169. C. Sun, P. Wang, H. Wang, C. Xu, J. Zhu et al., Defect engineering of molybdenum disulfide through ion irradiation to boost hydrogen evolution reaction performance. Nano Res. 12(7), 1613-1618 (2019). https://doi.org/10.1007/s1227 4-019-2400-1

170. Y. Liu, H. Nan, X. Wu, W. Pan, W. Wang et al., Layer-bylayer thinning of $\mathrm{MoS}_{2}$ by plasma. ACS Nano 7(5), 42024209 (2013). https://doi.org/10.1021/nn400644t

171. S. Mignuzzi, A.J. Pollard, N. Bonini, B. Brennan, I.S. Gilmore, M.A. Pimenta, D. Richards, D. Roy, Effect of disorder on raman scattering of single-layer $\mathrm{MoS}_{2}$. Phys. Rev. B 91(19), 195411 (2015). https://doi.org/10.1103/PhysRevB.91.195411

172. T.S. Sreeprasad, P. Nguyen, N. Kim, V. Berry, Controlled, defect-guided, metal-nanoparticle incorporation onto $\mathrm{MoS}_{2}$ via chemical and microwave routes: electrical, thermal, and structural properties. Nano Lett. 13(9), 4434-4441 (2013). https:// doi.org/10.1021/n1402278y

173. X. Zhang, T.H. Choudhury, M. Chubarov, Y. Xiang, B. Jariwala et al., Diffusion-controlled epitaxy of large area coalesced $\mathrm{WSe}_{2}$ monolayers on sapphire. Nano Lett. 18(2), 1049-1056 (2018). https://doi.org/10.1021/acs.nanolett.7b04521

174. H. Lin, Q. Zhu, D. Shu, D. Lin, J. Xu, X. Huang, W. Shi, X. Xi, J. Wang, L. Gao, Growth of environmentally stable transition metal selenide films. Nat. Mater. 18, 602-607 (2019). https:// doi.org/10.1038/s41563-019-0321-8

175. T. Daeneke, P. Atkin, R. Orrell-Trigg, A. Zavabeti, T. Ahmed et al., Wafer-scale synthesis of semiconducting SnO monolayers from interfacial oxide layers of metallic liquid tin. ACS Nano 11(11), 10974-10983 (2017). https://doi.org/10.1021/acsna no. $7 \mathrm{~b} 04856$

176. B. Radisavljevic, A. Radenovic, J. Brivio, V. Giacometti, A. Kis, Single-layer $\mathrm{MoS}_{2}$ transistors. Nat. Nanotechnol. 6(3), 147-150 (2011). https://doi.org/10.1038/nnano.2010.279

177. L. Tang, C. Teng, Y. Luo, U. Khan, H. Pan et al., Confined van der waals epitaxial growth of two-dimensional large singlecrystal $\mathrm{In}_{2} \mathrm{Se}_{3}$ for flexible broadband photodetectors. Research 2019, 10 (2019). https://doi.org/10.1155/2019/2763704

178. J.L.M. Östling, Scalable fabrication of 2D Semiconducting crystals for future electronics. Electronics 4(4), 1033-1061 (2015). https://doi.org/10.3390/electronics4041033

179. H. Li, G. Lu, Y. Wang, Z. Yin, C. Cong et al., Mechanical exfoliation and characterization of single- and few-layer nanosheets of $\mathrm{WSe}_{2}, \mathrm{TaS}_{2}$, and $\mathrm{TaSe}_{2}$. Small 9(11), 1974-1981 (2013). https://doi.org/10.1002/smll.201202919

180. A. Castellanos-Gomez, M. Barkelid, A.M. Goossens, V.E. Calado, H.S.J. van der Zant, G.A. Steele, Laser-thinning of $\mathrm{MoS}_{2}$ : on demand generation of a single-layer semiconductor. Nano Lett. 12(6), 3187-3192 (2012). https://doi.org/10.1021/ $\mathrm{nl} 301164 \mathrm{v}$

181. R. Yue, Y. Nie, L.A. Walsh, R. Addou, C. Liang et al., Nucleation and growth of $\mathrm{Wse}_{2}$ : enabling large grain transition metal dichalcogenides. 2D Mater. 4(4), 045019 (2017). https://doi. org/10.1088/2053-1583/aa8ab5

182. K. Kalantar-Zadeh, J. Tang, T. Daeneke, A.P. O’Mullane, L.A. Stewart et al., Emergence of liquid metals in nanotechnology. ACS Nano 13(7), 7388-7395 (2019). https://doi.org/10.1021/ acsnano.9b04843

183. A. Arash, T. Ahmed, A. Govind Rajan, S. Walia, F. Rahman et al., Large-area synthesis of $2 \mathrm{D} \mathrm{MoO}_{3-\mathrm{X}}$ for enhanced optoelectronic applications. 2D Mater. 6(3), 035031 (2019). https ://doi.org/10.1088/2053-1583/ab1114

184. F. Rahman, A. Zavabeti, M.A. Rahman, A. Arash, A. Mazumder et al., Dual selective gas sensing characteristics of $2 \mathrm{D} \mathrm{A}-\mathrm{MoO}_{3-\mathrm{X}}$ via a facile transfer process. ACS Appl. Mater. Interfaces 11(43), 40189-40195 (2019). https://doi. org/10.1021/acsami.9b11311 